


\section{Risk factors for violent behavior in people with psychotic disorders}

Risicofactoren voor gewelddadig gedrag bij mensen met een psychotische stoornis

(met een samenvatting in het Nederlands)

\section{Proefschrift}

ter verkrijging van de graad van doctor aan de Universiteit Utrecht op gezag van de rector magnificus, prof.dr. H.R.B.M. Kummeling, ingevolge het besluit van het college voor promoties in het openbaar te verdedigen op dinsdag 15 juni 2021 des ochtends te 10.15 uur

door

Jelle Lamsma

geboren op 10 september 1982

te Zeist 


\section{Promotoren:}

Prof.dr. W. Cahn

Prof.dr. S.B. Fazel 


\section{Table of contents}

Chapter 1

General introduction

Chapter 2 Violence in psychosis: conceptualizing its causal relationship with risk factors

Chapter 3

Use of illicit substances and violent behavior in psychotic disorders: two nationwide casecontrol studies and meta-analyses

Chapter 4

Cognition and violent behavior in psychotic disorders: a nationwide case-control study

Chapter 5

Risk factors for violence before and after the onset of schizophrenia spectrum disorders: a Dutch nationwide case-control study

Chapter 6 Structural brain correlates of interpersonal violence: systematic review and voxel-based meta-analysis of neuroimaging studies

Chapter $7 \quad$ General discussion 



\section{Chapter 1}

General introduction 


\section{Violence $^{*}$}

Violent behavior is increasingly recognized as a global public health problem ${ }^{1}$. It is in the top 10 causes of death among people under $45^{2}$ and in the top 20 for years of life lost across all ages $^{3}$. Each year, around 500,000 people die from homicide and many more experience nonfatal violence ${ }^{4}$.

Violence has a large impact on the economy. The annual costs of concomitant medical care, productivity losses and criminal justice processes are estimated at $€ 1.40$ trillion $^{5}$. However, the most serious consequence of violence - the suffering of victims - cannot be measured directly.

\section{Violence in psychotic disorders}

Psychotic disorders are a group of mental disorders that cause disturbances in perception, thinking, emotion and behavior ${ }^{6,7}$. Examples are schizophrenia, schizoaffective disorder and delusional disorder ${ }^{6}$. Symptoms include delusions (unusual beliefs that are held despite compelling evidence to the contrary), hallucinations (sensory experiences that occur in the absence of external stimuli), disorganized thinking (inferred from speech that is difficult to understand), diminished emotional expression, and loss of motivation ${ }^{6,7}$. Heritability estimates of the susceptibility to develop a psychotic disorder are as high as $80 \%^{8}$. Other risk factors are perinatal complications, stressful life events and heavy cannabis use during adolescence ${ }^{9}$. Psychotic disorders are a leading cause of disability worldwide ${ }^{10}$ and, despite affecting less than $1 \%$ of the population ${ }^{11}$, among the most expensive mental disorders ${ }^{12}$.

\footnotetext{
* This dissertation focuses on interpersonal violence, defined as the intentional use of physical force to harm another person. Self-directed and collective violence are excluded.
} 
Over the past 30 years, a robust association has been established between psychotic disorders and violent behavior. Based on convenience samples, it has been estimated that between $10 \%$ and $25 \%$ of patients act violently at some point in their lives ${ }^{13-15}$. Recently, more precise estimates have been provided by register-based studies. One such study, using data on all individuals diagnosed with a psychotic disorder in Sweden between 1972 and 2009 ( $N$ $=24,297)$, found that the lifetime prevalence of violent crime was $16 \%{ }^{16}$. Risk estimates vary widely, with studies reporting odds of violence in patients that are up to seven times higher than in the general population ${ }^{17-19}$. In a meta-analysis of 20 studies involving 18,423 cases and $1,714,904$ general population controls, the odds ratio of violence was 5.5 (95\% confidence interval $[\mathrm{Cl}]$ 4.1-7.5) in schizophrenia and 4.9 (95\% Cl 3.6-6.6) in other psychotic disorders ${ }^{20}$. Subsequent studies have shown that such risk remains when patients are compared with their unaffected siblings ${ }^{21,22}$, suggesting a causal relationship. The population attributable fraction of violence in psychotic disorders has been estimated at $5 \%{ }^{23}$.

Several factors have been identified that increase the risk of violent behavior in psychotic disorders. In a meta-analysis, containing information for a total of 45,533 cases from 110 studies, the strongest risk factors were: parental crime, childhood maltreatment, previous violence, substance use disorder (SUD), impulsivity, treatment nonadherence, and lack of illness insight ${ }^{24}$. With respect to symptoms, delusions ${ }^{25,26}$ and hallucinations ${ }^{13,27}$ have most frequently shown to be associated with violent behavior. Only four risk factors (treatment nonadherence, poor insight, and symptoms of delusions and hallucinations) are specific to psychotic disorders; the others also apply in the general population ${ }^{28}$.

However, there are four important areas of uncertainty. First, causal mechanisms are poorly understood. The main reason for this is that almost all studies have been retrospective in design ${ }^{29}$. Second, it is unclear to what extent different types of illicit substances, as defined by their psychopharmacological properties, and substance use below diagnostic thresholds for SUD are related to violence. These questions have a theoretical rationale in that the psychopharmacological properties of substances modify violence risk in the general population ${ }^{30}$ and people with psychotic disorders are highly sensitive to the harmful effects of substances $^{31}$. The few studies of cannabis $\mathrm{s}^{32-34}$ and stimulants $s^{35-37}$ have used surrogate outcomes, 
such as hostility or aggression, while none have investigated depressants or hallucinogens. Third, the role of cognition is largely unknown. Cognitive impairment is a hallmark of psychotic disorders ${ }^{38}$. Furthermore, it has been linked to violent behavior in the general population ${ }^{39-41}$ and adverse outcomes in patients, including low educational attainment ${ }^{42}$, SUD ${ }^{43}$ and rehospitalization ${ }^{44}$. Relevant studies have been limited by composite measures of cognitive functions or small selections thereof ${ }^{45-49}$. Finally, considerable debate exists as to whether risk factors are different for violent behavior that develops before (VBO) rather than after (VAO) the onset of illness ${ }^{50}$. It is plausible that risk factors for VBO are similar as for violence in the general population and that risk factors for VAO are primarily illness related. To my knowledge, these hypotheses have never been tested.

A common limitation of previous studies, which adds to the uncertainties described above, is the use of inpatient samples ${ }^{24}$. Such samples are likely selective, as many individuals with a psychotic disorder are never hospitalized ${ }^{51}$. What is more, inpatients may differ from outpatients on risk factors for violence. In particular, rates of SUD ${ }^{52}$ and symptom severity ${ }^{53}$ tend to be higher in the former.

Besides harm to victims and the economic costs already mentioned, violence in psychotic disorders has two other consequences. First, it contributes to stigma. Although most people with psychotic disorders are never violent, a large section of the public perceives them as such ${ }^{54}$. This perception, fueled by extensive news coverage ${ }^{55}$ and stereotyped portrayals in fiction ${ }^{56}$, is an important cause of stigma ${ }^{57}$. Stigma, in turn, adversely affects self-esteem ${ }^{58}$, employment opportunities ${ }^{59}$, social relationships ${ }^{60}$ and treatment adherence ${ }^{61}$. Second, costs are imposed on the mental healthcare system in the form of hospitalization. Forensic care in secure hospital settings is particularly expensive. In the Netherlands, as in the rest of Western Europe, the number of forensic beds has increased steadily since $1990^{62}$. There are now almost 1,400 places for the compulsory treatment of mentally ill offenders, called 'terbeschikkingstelling' $(\mathrm{TBS})^{63}$. About a third of the patients in TBS institutions, which are usually at near full capacity, are diagnosed with a psychotic disorder ${ }^{63}$. The annual per-person costs of TBS average $€ 190,000$, more than twice as much as prison ${ }^{64}$. The main justification for such high costs is the prevention of recidivism ${ }^{65}$. 
Knowledge about violent behavior in psychotic disorders is necessary to develop strategies for its prediction, prevention and management. This knowledge is lacking in current practice. Risk assessment tools are designed for use in either the general population or any mental disorder, and the little evidence available for their usefulness in psychotic disorders is weak ${ }^{66}$. The same is true for psychological interventions ${ }^{67}$. However, there is robust evidence that antipsychotics reduce violence ${ }^{68,69}$.

\section{Regional grey matter volume and violence}

Neuroimaging has great potential to provide insight into the neurobiology of violent behavior $^{70}$. Being noninvasive and allowing the measurement of brain structure or activity in healthy individuals, it has important advantages over lesion studies ${ }^{71}$. The most common index of structure is grey matter (GM) volume. GM consists mainly of neuronal and glial cell bodies ${ }^{72}$. Therefore, the GM volume of a brain region is taken to reflect its integrity ${ }^{73}$.

Brain regions typically implicated in violence are the orbitofrontal cortex, dorsolateral prefrontal cortex, anterior cingulate cortex, insula, amygdala, hippocampus and striatum ${ }^{74-76}$. Reduced GM volume in these regions is assumed to cause violent behavior through disturbances in emotional regulation, reward and avoidance learning, and decision making ${ }^{74}$.

Loss of GM in psychotic disorders is widespread but most pronounced in the frontal and temporal lobes, hippocampus and amygdala ${ }^{77}$. Additionally, it is related to relapse ${ }^{78}$, cognitive deficits $^{79}$ and poor social functioning ${ }^{80}$. For these reasons, it seems likely that reductions in regional GM volume underlie at least some violence in psychotic disorders. Only a few studies have investigated this. The largest was published last year. This study pooled data for 34 regions in the cerebral cortex of 902 cases from 10 research centers across the world. The findings showed that thinning ${ }^{*}$ of the temporal gyrus and midcingulate cortex was associated with

\footnotetext{
${ }^{*}$ In the cerebral cortex, GM volume is the product of thickness and surface area ${ }^{81}$.
} 
both hostility and a composite measure of delusions, hallucinations and disorganized thinking around the time of the $\operatorname{scan}^{82}$. Hostility is, of course, not the same as violence. Other studies have used small samples of inpatients and reported on mostly different brain regions ${ }^{83}$.

Studies in the general population or other psychiatric disorders may also contribute to our understanding of the neurobiological correlates of violence in psychotic disorders. There have been over 30 such studies. However, inconsistent results and variation in anatomical terminology hamper interpretation. Reviews have been limited to small numbers of selected brain regions and have included personality disorders, such as psychopathy and antisocial personality disorder, for which violence is not a required diagnostic criterion ${ }^{84-86}$.

\section{Aims and outline of this dissertation}

This dissertation will answer five questions.

(1) What mechanisms possibly underlie the observed relationships between risk factors and violence in psychotic disorders (chapter 2)?

(2) What types of illicit substances and severity levels of their use are associated with violent behavior in psychotic disorders (chapter 3)?

(3) What cognitive functions are related to violence in psychotic disorders (chapter 4)?

(4) To what extent do risk factors for VBO and VAO differ (chapter 5)?

(5) What brain regions, in terms of GM volume, are related to violent behavior (chapter 6)?

Question 1 will be answered based on a narrative review of the literature. To answer questions 2-5, I will use data from the Genetic Risk and Outcome of Psychosis project in the Netherlands. I will additionally draw on data collected for the National Development and Impact of Early Intervention Services project in the United Kingdom to answer question 3. These data largely overcome the issue of sampling bias, as patients were recruited from a wide variety of 
care settings across each country and independently of violent behavior. A systematic review of neuroimaging studies will be conducted to answer question 6 . 


\section{References}

1. Krug E, Mercy J, Dahlberg L, et al. The world report on violence and health. Lancet 2002; 360: 1038-1088.

2. Curry P, Ramaiah R, Vavilala MS. Current trends and update on injury prevention. Int J Crit IIIn Inj Sci 2011; 1: 57-65.

3. GBD 2017 Causes of Death Collaborators. Global, regional, and national age-sex-specific mortality for 282 causes of death in 195 countries and territories, 1980-2017: a systematic analysis for the global burden of disease study 2017. Lancet 2018; 392: 17361788.

4. World Health Organization. Global status report on violence prevention 2014. WHO, 2014.

5. Hoeffler A. What are the costs of violence? Politics Philos Econ 2017; 16: 422-445.

6. American Psychiatric Association. Diagnostic and Statistical Manual of Mental Disorders (5 $5^{\text {th }}$ edition). American Psychiatric Publishing, 2013.

7. World Health Organization. The ICD-10 classification of mental and behavioral disorders: clinical descriptions and diagnostic guidelines. WHO, 1992.

8. Strobl EV, Eack SM, Swaminathan S, et al. Predicting the risk of psychosis onset: advances and prospects. Early Interv Psychiatry 2012; 6: 368-379.

9. Mäki P, Veijola J, Jones PB, et al. Predictors of schizophrenia: a review. Br Med Bull 2005; 73-74: 1-15.

10. Rosenheck R, Leslie $D$, Keefe $R$, et al. Barriers to employment for people with schizophrenia. Am J Psychiatry 2006; 163: 411-417.

11. Moreno-Küstner B, Martín C, Pastor L. Prevalence of psychotic disorders and its association with methodological issues: a systematic review and meta-analyses. PloS One 2018; 13: e0195687.

12. Chong HI, Teoh SL, Wu DB, et al. Global economic burden of schizophrenia: a systematic review. Neuropsychiatr Dis Treat 2016; 2: 357-373.

13. Swanson JW, Swartz MS, Van Dorn RA, et al. A national study of violent behavior in persons with schizophrenia. Arch Gen Psychiatry 2006; 63: 490-499. 
14. Corrigan PW, Watson AC. Findings from the National Comorbidity Survey on the frequency of violent behavior in individuals with psychiatric disorders. Psychiatry Res 2005; 136: 153-162.

15. Mullen PE, Burgess $P$, Wallace $C$, et al. Community care and criminal offending in schizophrenia. Lancet 2000; 355: 614-617.

16. Fazel S, Wolf A, Palm C, et al. Violent crime, suicide, and premature mortality in patients with schizophrenia and related disorders: a 38-year total population study in Sweden. Lancet Psychiatry 2014; 1: 44-54.

17. Hodgins S, Alderton J, Cree A, et al. Aggressive behaviour, victimization and crime among severely mentally ill patients requiring hospitalization. Br J Psychiatry 2007; 191: 343350.

18. Elbogen $E$, Johnson $S$. The intricate link between violence and mental disorder: results from the National Epidemiologic Survey on Alcohol and Related Conditions. Arch Gen Psychiatry 2009; 66: 152-161.

19. Fazel S, Grann M, Carlström E, et al. Risk factors for violent crime in schizophrenia: a national cohort study of 13,806 patients. J Clin Psychiatry 2009; 70: 362-369.

20. Fazel S, Gulati G, Linsell L, et al. Schizophrenia and violence: systematic review and metaanalysis. PloS Med 2009; 6: e100012.

21. Sariaslan A, Larsson H, Fazel S. Genetic and environmental determinants of violence risk in psychotic disorders: a multivariate quantitative genetic study of 1.8 million Swedish twins and siblings. Mol Psychiatry 2016; 21: 1251-1256.

22. Fleischman A, Werbeloff N, Yoffe R, et al. Schizophrenia and violent crime: a populationbased study. Psychol Med 2014; 44: 3051-3057.

23. Fazel S, Smith EN, Chang Z, et al. Risk Factors for interpersonal violence: an umbrella review of meta-analyses. Br J Psychiatry 2018; 213: 609-614.

24. Witt K, Van Dorn RA, Fazel S. Risk factors for violence in psychosis: systematic review and meta-regression analysis of 110 studies. PloS One 2013; 8: e55942.

25. Coid JW, Ullrich S, Kallis C, et al. The relationship between delusions and violence: findings from the East London First Episode Psychosis Study. JAMA Psychiatry 2013; 70: 465-471.

26. Ullrich S, Keers R, Coid JW. Delusions, anger, and serious violence: new findings from the MacArthur Violence Risk Assessment Study. Schizophr Bull 2014; 40: 1174-1178. 
27. Fresán A, Apiquian R, de la Fuente-Sandoval C, et al. Violent behavior in schizophrenic patients: relationship with clinical symptoms. Aggress Behav 2005; 31: 511-520.

28. Farrington DP, Gaffney H, Ttofi MM. Systematic reviews of explanatory risk factors for violence, offending, and delinquency. Aggress Viol Behav 2017; 33: 24-36.

29. Appelbaum PS. In search of a new paradigm for research on violence and schizophrenia. Am J Psychiatry 2019; 176: 677-679.

30. Smith PH, Homish GG, Leonard KE, et al. Intimate partner violence and specific substance use disorders: findings from the National Epidemiologic Survey on Alcohol and Related Conditions. Psychol Addict Behav 2012; 26: 236-245.

31. Gregg L, Barrowclough C, Haddock G. Reasons for increased substance use in psychosis. Clin Psychol Rev 2007; 27: 494-510.

32. Koen L, Kinnear CJ, Corfield VA, et al. Violence in male patients with schizophrenia: risk markers in a South African population. Aust N Z J Psychiatry 2004; 38: 254-259.

33. Moulin V, Baumann P, Gholamrezaee M, et al. Cannabis, a significant risk factor for violent behavior in the early phase psychosis. Two patterns of interaction of factors increase the risk of violent behavior: cannabis use disorder and impulsivity; cannabis use disorder, lack of insight and treatment adherence. Front Psychiatry 2008; 9: 294.

34. Oluwoye O, Monroe-DeVita M, Burduli E, et al. Impact of tobacco, alcohol and cannabis use on treatment outcomes among patients experiencing first episode psychosis: data from the national RAISE-ETP study. Early Interv Psychiatry 2019; 13: 142-146.

35. Bell M, Greig T, Gill P, et al. Work rehabilitation and patterns of substance use among persons with schizophrenia. Psychiatr Serv 2002; 53: 63-69.

36. Miles $\mathrm{H}$, Johnson $\mathrm{S}$, Amponsah-Afuwape $\mathrm{S}$, et al. Characteristics of subgroups of individuals with psychotic illness and a comorbid substance use disorder. Psychiatr Serv 2003; 54: 554-561.

37. Harris AW, Large MM, Redoblado-Hodge A, et al. Clinical and cognitive associations with aggression in the first episode of psychosis. Aust N Z J Psychiatry 2010; 44: 85-93.

38. Kahn RS, Keefe RS. Schizophrenia is a cognitive illness: time for a change in focus. JAMA Psychiatry 2013; 70: 1107-1112.

39. Ogilvie JM, Stewart AL, Chan RC, et al. Neuropsychological measures of executive function and antisocial behavior: a meta-analysis. Criminology 2011; 49: 1063-1107. 
40. Hoaken PN, Allaby DB, Earle J. Executive cognitive functioning and the recognition of facial expressions of emotion in incarcerated violent offenders, non-violent offenders, and controls. Aggress Behav 2007; 33: 412-421.

41. Nyline B, Softas-Nall L, Peterson E. Inaccuracies in facial recognition of fear and sadness for male domestic violence offenders. Open J Soc Sci 2018; 6: 37-51.

42. Keefe RS. Should cognitive impairment be included in the diagnostic criteria for schizophrenia? World Psychiatry 2008; 7: 22-28.

43. Eack SM, Hogarty SS, Greenwald DP, et al. Cognitive enhancement therapy in substance misusing schizophrenia: results of an 18-month feasibility trial. Schizophr Res 2015; 61: 478-483.

44. Harvey PD, Loewenstein DA, Czaja SJ. Hospitalization and psychosis: influences on the course of cognition and everyday functioning in people with schizophrenia. Neurobiol Dis 2013; 53: 18-25.

45. Barkataki I, Kumari V, Das M, et al. A neuropsychological investigation into violence and mental illness. Schizophr Res 2005; 74: 1-13.

46. Fullam RS, Dolan MC. Executive function and in-patient violence in forensic patients with schizophrenia. Br J Psychiatry 2008; 193: 247-253.

47. Krakowski MI, De Sanctis P, Foxe JJ, et al. Disturbances in response inhibition and emotional processing as potential pathways to violence in schizophrenia: a high-density event-related potential study. Schizophr Bull 2016; 42: 963-974.

48. Silver H, Goodman C, Knoll G, et al. Schizophrenia patients with a history of severe violence differ from nonviolent schizophrenia patients in perception of emotions but not cognitive function. J Clin Psychiatry 2005; 66: 300-308.

49. Frommann N, Stroth S, Brinkmeyer J, et al. Facial affect recognition performance and event-related potentials in violent and non-violent schizophrenia patients Neuropsychobiology 2013; 68: 139-145.

50. Hodgins S. Aggressive behavior among persons with schizophrenia and those who are developing schizophrenia: attempting to understand the limited evidence on causality. Schizophr Bull 2017; 43: 1021-1026.

51. Walsh E, Buchanan A, Fahy T. Violence and schizophrenia: examining the evidence. Br J Psychiatry 2002; 180: 490-495. 
52. Winklbaur B, Ebner N, Sachs G, et al. Substance abuse in patients with schizophrenia. Dialogues Clin Neurosci 2006; 8: 37-43.

53. Millier A, Horváth $M, M a F$, et al. Healthcare resource use in schizophrenia, EuroSC findings. J Mark Access Health Policy 2017; 5: 1372027.

54. Pescosolido BA, Manago B, Monahan J. Evolving public views on the likelihood of violence from people with mental illness: stigma and its consequences. Health Aff 2019; 38: 17351743.

55. Gwarjanski AR, Parrott S. Schizophrenia in the news: the role of news frames in shaping online reader dialogue about mental illness. Health Commun 2018; 33: 954-961.

56. Owen PR. Portrayals of schizophrenia by entertainment media: a content analysis of contemporary movies. Psychiatr Serv 2012; 63: 655-659.

57. Torrey EF. Stigma and violence: isn't it time to connect the dots? Schizophr Bull 2011; 37: 892-896.

58. Chronister J, Chou CC, Liao HY. The role of stigma coping and social support in mediating the impact of societal stigma on internalized stigma, mental health recovery and quality of life among persons with serious mental illness. J Community Psychol 2013; 41: 582600.

59. Brouwers EP. Social stigma is an underestimated contributing factor to unemployment in people with mental illness or mental health issues: position paper and future directions. BMC Psychol 2020; 8: 36.

60. Hoftman GD. The burden of mental illness beyond clinical symptoms: impact of stigma on the onset and course of schizophrenia spectrum disorders. Am J Psychiatry 2017; 11: $5-7$.

61. Kane JC, Elafros MA, Murray SM, et al. A scoping review of health-related stigma outcomes for high-burden diseases in low- and middle-income countries. BMC Med 2019; 17: 17 .

62. Chow WS, Priebe S. How has the extent of institutional mental healthcare changed in Western Europe? Analysis of data since 1990. BMJ Open 2016; 29: e010188.

63. Dienst Justitiële Inrichtingen. DJI in getal: 2013-2017. DJI, 2017.

64. Nagtegaal MH, Goethals K, Meynen G. De tbs-maatregel: kosten en baten in perspectief. Tijdschr voor Psychiatr 2016; 58: 739-745. 
65. Wolf A, Fanshawe TR, Sariaslan A, et al. Prediction of violent crime on discharge from secure psychiatric hospitals: a clinical prediction rule (FoVOx). Eur Psychiatry 2018; 47: 88-93.

66. Singh JP, Serper M, Reinharth J, et al. Structured assessment of violence risk in schizophrenia and other psychiatric disorders: a systematic review of the validity, reliability, and item content of 10 available instruments. Schizophr Bull 2011; 37: 899912.

67. Maclnnes D, Masino S. Psychological and psychosocial interventions offered to forensic mental health inpatients: a systematic review. BMJ Open 2019; 20: e024351.

68. Faay MD, Czobor P, Sommer IE. Efficacy of typical and atypical antipsychotic medication on hostility in patients with psychosis-spectrum disorders: a review and meta-analysis. Neuropsychopharmacology 2018; 43: 2340-2349.

69. Fazel S, Zetterqvist J, Larsson $\mathrm{H}$, et al. Antipsychotics, mood stabilisers, and risk of violent crime. Lancet 2014; 384: 1206-1214.

70. Poldrack RA, Monahan J, Imrey PB, et al. Predicting violent behavior: what can neuroscience add? Trends Cogn Sci 2018; 22: 111-123.

71. Rordan C, Karnath HO. Using human brain lesions to infer function: a relic from a past era in the fMRI age? Nat Rev Neurosci 2004; 5: 813-819.

72. Snell RS. Clinical neuroanatomy. Lippincott Williams \& Wilkins, 2010.

73. Ohtani T, Nestor PG, Bouix S, et al. Medial frontal white and gray matter contributions to general intelligence. PloS One 2014; 9: e112691.

74. Raine A. Genes to brain to antisocial behavior. Curr Dir Psychol Sci 2008; 17: 323-328.

75. Craig AD. How do you feel - now? The anterior insula and human awareness. Nat Rev Neurosci 2009; 10: 59-70.

76. Anderson NE, Kiehl KA. The psychopath magnetized: insights from brain imaging. Trends Cogn Sci 2010; 16: 52-60.

77. Haijma SV, van Haren N, Cahn W, et al. Brain volumes in schizophrenia: a meta-analysis in over 18000 subjects. Schizophr Bull 2013; 39: 1129-1138.

78. Andreasen NC, Liu D, Ziebell S, et al. Relapse duration, treatment intensity, and brain tissue loss in schizophrenia: a prospective longitudinal MRI study. Am J Psychiatry 2013; 170: 609-615. 
Chapter 1

79. Kahn RS, Sommer IE. The neurobiology and treatment of first-episode schizophrenia. Mol Psychiatry 2015; 20: 84-89.

80. Cahn W, van Haren NE, Hulshoff Pol HW, et al. Brain volume changes in the first year of illness and 5-year outcome of schizophrenia. Br J Psychiatry 2006; 189: 381-382.

81. Winkler AM, Kochunov P, Blangero J, et al. Cortical thickness or grey matter volume? The importance of selecting the phenotype for imaging genetics studies. Neuroimage 2010; 53: 1135-1146.

82. Wong TJ, Radua J, Pomarol-Clotet E, et al. An overlapping pattern of cerebral cortical thinning is associated with both positive symptoms and aggression in schizophrenia via the ENIGMA consortium. Psychol Med 2019; 16: 1-12.

83. Fjellvang M, Grøning L, Haukvik UK. Imaging violence in schizophrenia: a systematic review and critical discussion of the MRI literature. Front Psychiatry 2018; 9: 333.

84. Blair RJ. Neuroimaging of psychopathy and antisocial behavior: a targeted review. Curr Psychiatry Rep 2010; 12: 76-82.

85. Yang $\mathrm{Y}$, Raine A. Prefrontal structural and functional brain imaging findings in antisocial, violent, and psychopathic individuals: a meta-analysis. Psychiatry Res Neuroimaging 2009; 174: 81-88.

86. Brower MC, Price BH. Neuropsychiatry of frontal lobe dysfunction in violent and criminal behaviour: a critical review. J Neurol Neurosurg Psychiatry 2001; 71: 720-726. 


\section{Chapter 2}

Violence in psychosis: conceptualizing its causal relationship with risk factors

Lamsma J

Harte JM

Aggress Violent Behav 2015; 24: 75-82. 
Chapter 2

\section{Abstract}

\section{Background}

While statistically robust, the association between psychosis and violence remains causally unexplained.

\section{Methods}

We conducted a structured narrative review of relevant studies published between 1990 and 2013 , found via online databases and bibliographies. Both reviews and empirical studies were included. No restrictions were applied to language, study design, sample characteristics and measurement of psychosis and violence. We excluded case reports and studies about selfharm. A final sample of 69 studies was used.

\section{Results}

The lack of knowledge regarding the causal relationship between psychosis and violence is partially due to methodological aspects of research. These aspects include study design, sampling, operationalization and confounding variables. Moreover, violence is the potential outcome of several interrelated risk factors: demographics; social factors; persecutory delusions; command hallucinations; comorbid antisocial personality pathology; substance use; inadequate insight; treatment nonadherence; and physiological factors. Forty-one possible causal pathways between these risk factors and violence are presented.

\section{Conclusion}

This review stimulates research by providing a theoretical framework, avenues for future investigation and methodological recommendations. Understanding violence in psychosis enhances its prevention and treatment, decreases stigma associated with psychosis and improves the patient's legal position. 


\section{Introduction}

Psychosis is a heterogeneous syndrome, characterized by delusions, hallucinations, diminished emotional expression and disorganized thinking and motor behavior ${ }^{1}$. These symptoms are often associated with a mental disorder such as schizophrenia, delusional disorder and schizoaffective disorder. Psychosis may also arise as a result of substance use and other medical conditions, including cerebrovascular, endocrine and neoplastic diseases, epilepsy and head trauma.

Since the 1990s, a large number of studies have found a positive and statistically robust association between psychosis and violence ${ }^{2-11}$. Prevalence rates of violent behavior among schizophrenia patients reported by recent large-scale prospective studies conducted in the United States and Sweden range between $9 \%$ and $20 \%{ }^{12-14}$. A meta-analysis of 204 studies found that the odds of violence in people diagnosed with psychosis are $49 \%$ to $68 \%$ higher relative to the odds of violence in people without such a diagnosis ${ }^{4}$. In another meta-analysis, psychoses were associated with an odds ratio for violence of 4.0 (95\% Confidence Interval [Cl] 3.0-5.3) in men and 7.90 (95\% Cl 4.0-15.4) in women ${ }^{5}$. The population-attributable risk for violence in schizophrenia has been estimated at less than $10 \%{ }^{11}$. Understanding violent behavior in psychotic patients is important for at least three reasons. First, it may reduce such behavior and the emotional and financial costs that come with it - by facilitating more effective prevention and treatment. Second, it may help to decrease stigma associated with the syndrome. Psychotic patients are often perceived as dangerous, while de facto only a small proportion of them act violently. Third, it serves to protect the civil rights of psychotic individuals by improving risk assessment in cases of civil and criminal commitment.

Numerous factors have been proposed to account for the increased risk of violence in psychosis, with examples being low socioeconomic standing, substance use, symptomatology and deficient insight. However, little is known about the mechanisms by which such risk factors may cause violent behavior in psychotic patients. In other words, studies have yet to move from correlation to causation. Moreover, prevailing hypotheses on this topic have not 
been the object of broad gauged review. The main purpose of the present study is therefore to provide an overview of possible causal pathways between the most replicated risk factors and violent behavior in psychosis. To this aim, a structured narrative review of the relevant literature is presented. Methodological issues of research in this field will be outlined first. The most replicated risk factors for violence in psychosis and possible causal pathways are subsequently examined. Finally, the study's limitations are discussed in conjunction with its implications for future research and practice.

\section{Methods}

A narrative review was conducted of studies found using an explicit search strategy with a set of inclusion criteria. A narrative review was chosen over a systematic review. Whereas a systematic review includes all traceable papers selected according to a strict protocol in order to evaluate findings in relation to methodological quality, a narrative review takes a more liberal approach useful for synthesizing the current body of knowledge in a certain research area and developing a theoretical framework ${ }^{16,17}$. The latter approach is consistent with the review's principal objective, which is to conceptualize the causal relationships between risk factors and violence in psychosis rather than to rigorously evaluate empirical evidence. Most risk factors presented here have been described in systematic reviews $4,5,9,15$.

\section{Search strategy}

The online databases of PubMed, PsycINFO, ScienceDirect and Google Scholar were searched for studies published between 1990 and 2013. Search terms covered psychosis (viz. "psychot*", "psychos*", "schizo*", "mental*"), violence (viz. "violen*”, "aggress*", "hosti*", "crim*", "offend*") and risk factors generically (viz. "risk*", "correlate*”, "variable*", "pre- 
dict*"). Additional searches were performed for specific risk factors (e.g. "gender", "hallucinations", "substance", "insight"). Other studies were found by manually searching relevant bibliographies.

\section{Inclusion criteria}

Both review articles and empirical studies were considered for inclusion, as long as they reported on risk factors for violence in psychosis. We chose to include and emphasize findings of reviews, as they are generally considered to be more valid than those of individual empirical studies. Moreover, authors may posit relevant hypotheses in either type of study. For the same reason, no restrictions were made relating to language, study design (i.e. cohort, casecontrol, cross-sectional), the type of sample used (i.e. prison, psychiatric, community), age of participants and measurement of psychosis (i.e. self-report, unstructured or structured interview) and violence (i.e. criminal records, case notes, collateral information, self-report). Case reports and studies using self-harm as the sole outcome measure were excluded. Studies were eligible irrespective of whether psychosis was associated with a mental disorder (e.g. schizophrenia, delusional disorder), substance use or another medical condition (e.g. brain tumor, epilepsy). Studies examining specific symptoms of psychosis (e.g. delusions, hallucinations) were also included. Following the structured search, 66 studies were selected. Three studies were added to provide hypotheses not presented in the literature obtained through the structured search. This brought the total number of studies used in this review to 69 .

\section{Results}

\section{Methodological issues in research on violence in psychosis}

The association between psychosis and violence has been found across different study designs and settings. However, several methodological aspects of research may affect the size 
of this association and thwart comparison between studies. The following aspects require attention: study design, sampling, operationalization of variables and confounding factors.

\section{Study design and sampling}

Since it is often impossible or unethical to expose subjects to relevant risk factors (e.g. neighborhood of residence, psychotic symptoms, substance use), most research on psychosis and violence is observational in nature and more subject to bias than experimental research. Three types of observational study design can be distinguished: cohort, case-control and cross-sectional. Establishing a causal relationship between two events is conditional on knowing their sequential order and temporal proximity. This is only possible with cohort studies in which variables of interest are measured chronologically in people who share a common characteristic (e.g. year of birth, diagnosis of schizophrenia) ${ }^{18}$. As subjects who demonstrate the outcome of interest are drawn from the same population as those who do not, confounding bias is minimized when comparing the two groups. The risk of sampling bias is reduced because participants are selected prior to demonstrating the outcome of interest. While convenient and efficient for studying a rare phenomenon such as psychosis, samples consisting of only discharged psychiatric patients could be selective, however. Findings may also be biased by attrition at follow-up, which is often related to the variables under study. Case-control studies compare two or more groups of people differing on an outcome with respect to attributes hypothesized to cause these group differences. This retrospective measurement gives rise to several kinds of bias. First, temporal bias, when it is uncertain whether the independent variable preceded and approximated the dependent variable in time. Second, biased reporting by patient or researcher if they are not blind which study group (i.e. case, control) the former belongs to. Finally, because selection of subjects is based on the outcome of interest, sampling is prone to bias ${ }^{18}$. It is also important that cases and controls do not systematically differ on one or more unmeasured qualities that confound the association between risk factors and violence. While it is unfeasible to find perfectly matching study groups or 
statistically control for all potential confounders, researchers should compare violent psychotic patients to nonviolent people with similar risk profiles, preferably siblings or other psychotic patients. However, the composition of comparison groups varies considerably across studies and often includes arguably less comparable subjects such as general population controls and nonpsychotic psychiatric patients ${ }^{4,11}$. Cross-sectional research does not allow inference of causality, as variables are measured within one study group at a single point in time ${ }^{18}$. Probably owing to the high cost and duration of cohort studies, most research on violence in psychosis involves case-control and cross-sectional studies. To illustrate, Fazel et al. ${ }^{5}$ identified in their meta-analysis of 20 studies only 3 cohort studies in which violence was measured after a diagnosis of schizophrenia or other psychosis was made. Clearly, more high-quality cohort studies are needed to advance this field of research.

Many studies draw samples from populations of patients institutionalized in correctional or psychiatric facilities ${ }^{4,11}$. These samples may be selective, since psychotic individuals who are convicted or hospitalized may be different from those who are not. At the same time, institutionalized patients are often underrepresented in community samples ${ }^{11}$. Thus, combined sampling is preferable.

\section{Definition and measurement of violence}

Definitions of violence vary widely. Some studies only count physical acts, which may range in seriousness from pushing to homicide, while others incorporate verbal behavior as well. Questionable definitions can also be found such as those including self-harm and vandalism. A number of studies fail to give any definition of violence ${ }^{3,4}$.

The most common data sources for the measurement of violence are criminal records, case notes, collateral information and self-report ${ }^{4,11}$. It is advisable to use these methods in combination because the information contained in each of them is often incomplete or selective: 
characteristics of the justice and mental health care systems, suspect and offense all determine the number and type of convictions; information in case notes is recorded only when relevant for the patient's criminal case, probation officer or clinician; the patient's family or friends may be ignorant of violent incidents or have conflicting interests; the reports of patients themselves may be affected by fear of negative consequences, social desirability and biased recall ${ }^{11}$.

\section{Definition and measurement of psychosis}

Psychosis is variably defined in studies. It is sometimes part of the broad concepts of Major Mental Disorder or Severe Mental Illness, which include different mental disorders such as schizophrenia and depression ${ }^{19-21}$. Other studies examine disorders of which psychotic symptoms are pathognomonic, such as schizophrenia or schizophreniform disorder ${ }^{22,23}$, or the syndrome of psychosis as a whole ${ }^{24}$. A last category of studies looks at specific symptoms of psychosis, most notably delusions and hallucinations ${ }^{25,26}$. Since psychosis is a heterogeneous syndrome with variable causes, symptom-level research seems most appropriate.

Methods for diagnosing psychosis range from self-report to unstructured and structured interviews ${ }^{3,4,11}$. Structured interviews are considered the most reliable and valid means of assessment. Because self-report measures are contingent on the patient's level of insight, defensiveness and malingering, their validity is often limited ${ }^{27}$. In addition, they typically do not contain questions regarding symptoms of psychosis ${ }^{4}$. While the quality of diagnoses based on unstructured interviews is improved when complemented with clinical observation, collateral information or the patient's medical history, they have poor inter-rater reliability and validity compared with structured interviews ${ }^{11}$. In structured interviews, the same information is collected from each patient with a standardized protocol whereby different interviewers are likely to reach similar conclusions. The diagnostic accuracy of structured interviews has been shown to be superior to that of unstructured interviews ${ }^{4,27}$. 


\section{Confounding factors}

It is possible that psychosis and violence are not causally related but the shared result of a third factor. Comorbid antisocial personality pathology and substance use have frequently been suggested as potential confounders ${ }^{28,29}$. Other oft-cited possibilities include the aforementioned methodological factors, deinstitutionalization and discrimination by health care or justice systems ${ }^{9,30,31}$. However, it is unlikely that these factors fully mediate the association between psychosis and violence, since this association has been observed across different time periods, locations and study designs ${ }^{6,32}$. A final possible confounding factor is publication bias. Unfortunately, few studies have investigated this possibility.

Possible causal pathways between risk factors and violence in psychosis

The following risk factors for violence in psychosis are most commonly cited in the literature: demographic factors; social factors; persecutory delusions and command hallucinations; comorbid antisocial personality pathology; substance use; inadequate insight; treatment nonadherence; and physiological factors.

\section{Demographic factors}

Demographic factors most frequently associated with violence in psychotic patients are male gender, young age, single status, homelessness, unemployment, low educational attainment, low socioeconomic status, belonging to an ethnic minority, past hospitalization for psychosis and past conviction of violent crime ${ }^{3,8,15,33}$. It is unclear how these factors causally relate to violence. Some may predispose patients toward violent behavior by reason of low self-control such as male gender and young age ${ }^{34}$. The effect of most demographic factors is probably indirect, since the association between violent behavior and psychosis has been shown to 
remain when these are controlled for ${ }^{7,8,30}$. Perhaps low socioeconomic status and unemployment reflect conditions of social disorganization. An adverse family history may account for low educational attainment. It is also possible that single status, homelessness, unemployment, educational problems and low socioeconomic standing are the consequence of habitual substance use, violence or severe psychotic symptomatology. Males tend to report more substance use than females, potentially mediating the relationship between gender and violence ${ }^{35}$. Symptom severity could explain past hospitalization. Past hospitalization may also be a sign of violent conduct, since this is often a reason or requisite for admission ${ }^{36}$. Past conviction of violent crime may represent a patient's violent disposition ${ }^{8}$. At the same time, mentally ill offenders are disproportionately represented in the criminal justice system ${ }^{30}$. Furthermore, familiarity with the authorities imaginably enhances the risk that someone will be convicted again or to a longer prison term.

\section{Social factors}

Psychotic patients who display violent behavior relatively often come from disadvantaged families with histories of criminality, substance use and physical or sexual maltreatment $^{13,15,31}$. These circumstances may lead to adult violence or substance use through processes of social learning or genetic influence ${ }^{13}$. Violent patients are likely to reside in socially disorganized neighborhoods, characterized by high rates of low-income residents, ethnic diversity, norms approving of violence, little social cohesion and a lack of both informal and formal social control ${ }^{30,31,37}$. This type of environment increases the likelihood of criminal victimization and other negative life events such as unemployment and divorce ${ }^{37}$. In addition, the patient's immediate social environment is often unsupportive ${ }^{38}$. Such stressful living conditions may increase the risk of violence directly or indirectly, through substance use or exacerbated psychopathology $30,37,39$. Hypothetically, they could also be the result of the patient's violent conduct or severe psychotic symptoms. 


\section{Persecutory delusions and command hallucinations}

To reliably infer a causal relationship, it is crucial not only to establish the mere presence of psychosis but also the onset and oscillation of symptoms in relation to violent behavior across a patient's life. After all, even if established before the occurrence of violence, a diagnosis of psychosis may have little predictive value when both events are temporally remote ${ }^{4}$. Moreover, it tells us nothing about the relative importance of specific symptoms. Unfortunately, this information is seldom recorded in studies. For that reason, theories implicating psychotic symptoms as causes of violence should be treated with caution.

In terms of psychotic symptoms, persecutory delusions and command hallucinations have usually shown the strongest correlation with violent behavior ${ }^{40-43}$. Mediation is likely, however, since both symptoms are common in patients ${ }^{44-46}$. There is an increasing body of evidence suggesting that delusional violence is often prompted by associated emotional responses such as anxiety, fear and anger ${ }^{47,48}$. However, it is important to note that these emotions could also be a sign of deficient illness insight ${ }^{49}$. Possible mediating features of command hallucinations include the perceived consequences of disobedience, the significance attributed to the command and the patient's attitude toward the voice's identity ${ }^{43,50}$. It is also possible that stress, as a consequence of violent behavior, elicits or exacerbates psychotic symptoms $\mathrm{s}^{4,49}$.

\section{Comorbid antisocial personality pathology}

Comorbid antisocial personality pathology has consistently been found to increase the risk of violence in psychosis. This particularly applies to diagnoses of conduct disorder (CD), antisocial personality disorder (APD) and psychopathy ${ }^{51-57}$. In broad terms, antisocial personality pathology is characterized by a disregard for social norms, deceitfulness, impulsivity, remorselessness and lack of empathy ${ }^{1}$. These personality characteristics may lead to violence in several ways. Firstly, they lower the patient's threshold to behave violently. They also make 
patients prone to substance use and treatment nonadherence ${ }^{52,56}$. Lastly, antisocial conduct may negatively affect the patient's social functioning ${ }^{3}$.

Comorbid antisocial personality pathology and symptoms of delusions and hallucinations have been found to predict violence independently, implying the existence of two distinct etiologies of violence in psychosis ${ }^{56,58}$. Patients with comorbid antisocial personality pathology usually display a lifelong pattern of persistent and versatile criminal conduct that commenced before the onset of illness. Violent behavior by these patients is thought to be predominantly associated with neurobiological abnormalities, substance use and histories of parental criminality and childhood maltreatment. In contrast, violence in patients without comorbid antisocial personality pathology is hypothesized to be primarily motivated by paranoid delusions and command hallucinations. These patients should, as a consequence, typically act violently during periods of florid psychosis ${ }^{54,56}$.

\section{Substance use}

Substance use is widely regarded as a major predictor for violence in psychosis ${ }^{4,5,12,59,60}$. There may be a number of ways in which substance use increases the risk of violent behavior. Through their psychopharmacological effects, substances reduce the patient's inhibitions ${ }^{6,39}$. Substance use may also have a detrimental impact on the patient's social support system ${ }^{61}$. Furthermore, the buying and selling of illegal drugs commonly takes place in criminogenic environments ${ }^{6}$. Certain drugs also aggravate psychotic symptoms. This is especially true for cannabis ${ }^{61}$. Paradoxically, the use of substances may simultaneously be a patient's attempt to alleviate psychotic symptoms or feelings of depression and anxiety ${ }^{61}$.

\section{Inadequate insight}

Insight in mental disorder is defined as the patient's recognition of illness, its symptoms and 
consequences, and the need for treatment ${ }^{49}$. Inadequate insight has frequently been suggested as a risk factor for violent behavior in psychotic patients ${ }^{62-64}$. However, studies on this subject are scarce and their results inconclusive ${ }^{49,65}$. Perhaps a strong belief that delusions or hallucinations are real increases the probability that the patient acts on them ${ }^{49}$. Denial of illness or the need for psychiatric care may also bring about violent behavior through nonadherence to treatment ${ }^{65}$.

\section{Treatment nonadherence}

Nonadherence to therapy and medication is associated with an elevated risk of violence in psychotic individuals ${ }^{15,51,62}$. There are several explanations for this finding. By refusing antipsychotic medication, psychotic symptoms do not diminish or even worsen ${ }^{30}$. Patients with persecutory delusions may be reluctant to comply with treatment due to fear of mental health professionals ${ }^{66}$. Lastly, treatment nonadherence appears to be associated with substance use $\mathrm{e}^{39,67}$. This association may be reciprocal, with nonadherence inducing self-medication and substance use interfering with treatment ${ }^{67}$.

\section{Physiological factors}

While still a relatively new area of research, there is some evidence to suggest that physiological factors are integral to the etiology of violent behavior in psychosis, particularly schizophrenia. The most consistent findings point to a low-activity polymorphism of the gene encoding catechol-O-methyltransferase (COMT), an enzyme involved in dopamine metabolism, serotonin hypofunction and frontal and temporal lobe abnormalities ${ }^{68-71}$. A positive association has also been observed between violence and medication side effects, most notably akathisia and neuroleptic-induced deficit syndrome ${ }^{31,68}$. These conditions are hypothesized to make the patient prone to violence by hampering the experience and recognition of emotions, lowering stress reactivity, increasing impulsivity and creating cognitive biases that favor 
aggressive responses to stressful situations ${ }^{70,72,73}$. Impairment of cognitive functions may also explain the patient's educational problems and susceptibility to substance use ${ }^{72,73}$. Overactive mesolimbic dopaminergic projections have been suggested to be responsible for the increased severity of delusions and hallucinations found in aggressive patients ${ }^{71}$. Finally, unpleasant side effects of medication may bring the patient to discontinue intake ${ }^{67}$.

\section{Summary}

Violent behavior in psychosis is etiologically complex. It may be caused by many interacting risk factors. Possible causal pathways between these risk factors and violence, as described in the preceding sections, are summarized below and schematically depicted in Figure 1 . The numbers assigned to each line correspond to those of each hypothesis. The arrows indicate direction. Causal pathways associated with comorbid antisocial personality pathology are represented by dashed lines. Dotted lines signify causal pathways associated with persecutory delusions and command hallucinations.

Certain demographic descriptors may be directly or indirectly related to violence in psychotic patients. Low self-control associated with being male or young of age predisposes the patient toward violent behavior (1). Males also tend to use substances more frequently than females (2). Low socioeconomic status and unemployment may reflect a high degree of social disorganization in the patient's area of residence (3), while an adverse family history potentially accounts for low educational attainment (4). Habitual substance use possibly explains the patient's single status, homelessness, unemployment, educational adversities and low economic standing (5). Single status, homelessness, unemployment, educational problems, low socioeconomic standing and past hospitalization may be caused by the patient's recurrent violent behavior (6) and severe psychotic symptoms (7). Besides being an indicator of violent conduct (8), previous convictions for violent offenses may reflect the patient's vulnerability for criminal justice system processing (9). 
Crime and maltreatment by family members could lead to later violence by way of social learning (10). Familial transmission of substance use is similarly conceivable (11). An adverse family history may also point to the influence of genetic factors (12). An adverse social environment, defined as living in a socially disorganized neighborhood and a lack of social support, may promote substance use (13), give rise to violence (14) and exacerbate psychotic symptoms (15). Alternatively, these circumstances may be a consequence of the patient's violent conduct (16) or severe psychotic symptomatology (17).

A patient's violent behavior may be propelled by certain features of persecutory delusions and command hallucinations (18). It is also possible that psychotic symptoms are aggravated or elicited by stress that accompanies acts of violence (19).

Comorbid antisocial personality pathology underlies an inclination toward violence (20), substance use (21) and treatment nonadherence (22). Persistent antisocial conduct could also explain a negative social environment (23). Antisocial personality pathology often emanates from an adverse family history (24) and neurobiological factors that are believed to be conducive to violence (25).

Substance use may cause violence directly through diminished behavioral control (26) or indirectly, either by placing the patient in a criminogenic and unsupportive social environment (27) or by aggravating psychotic symptoms (28). The patient may also use substances in an attempt to relieve psychotic symptoms (29).

Inadequate insight potentially leads to violence when the patient believes delusions or hallucinations are real (30) or to treatment nonadherence when the patient denies being ill or in need of psychiatric care (31).

Treatment nonadherence may be related to violent behavior in several ways. Psychotic symptoms continue or worsen without antipsychotic medication (32). In the absence of proper treatment, the patient may use substances as a means of self-medication (33). At the same time, substance use impedes successful treatment (34). Finally, persecutory delusions can 
Chapter 2

cause patients to be fearful and suspicious of mental health professionals and, as a consequence, oppose treatment (35).

Increased dopaminergic neurotransmission related to a low-activity polymorphism of the COMT gene and other forms of pathophysiology, such as serotonin hypofunction and abnormalities in the frontotemporal circuitry, may predispose the patient toward violent behavior (36) and substance use (37) by impairing emotional and cognitive functions. Cognitive deficits may also explain the patient's low educational attainment (38). A hyperactive mesolimbic dopamine system could underlie the presence of severe symptoms of delusions and hallucinations (39). Medication side effects may make the patient susceptible to violence (40) or cause intake discontinuation (41). 
Figure 1. Possible causal pathways between risk factors and violence in psychosis.

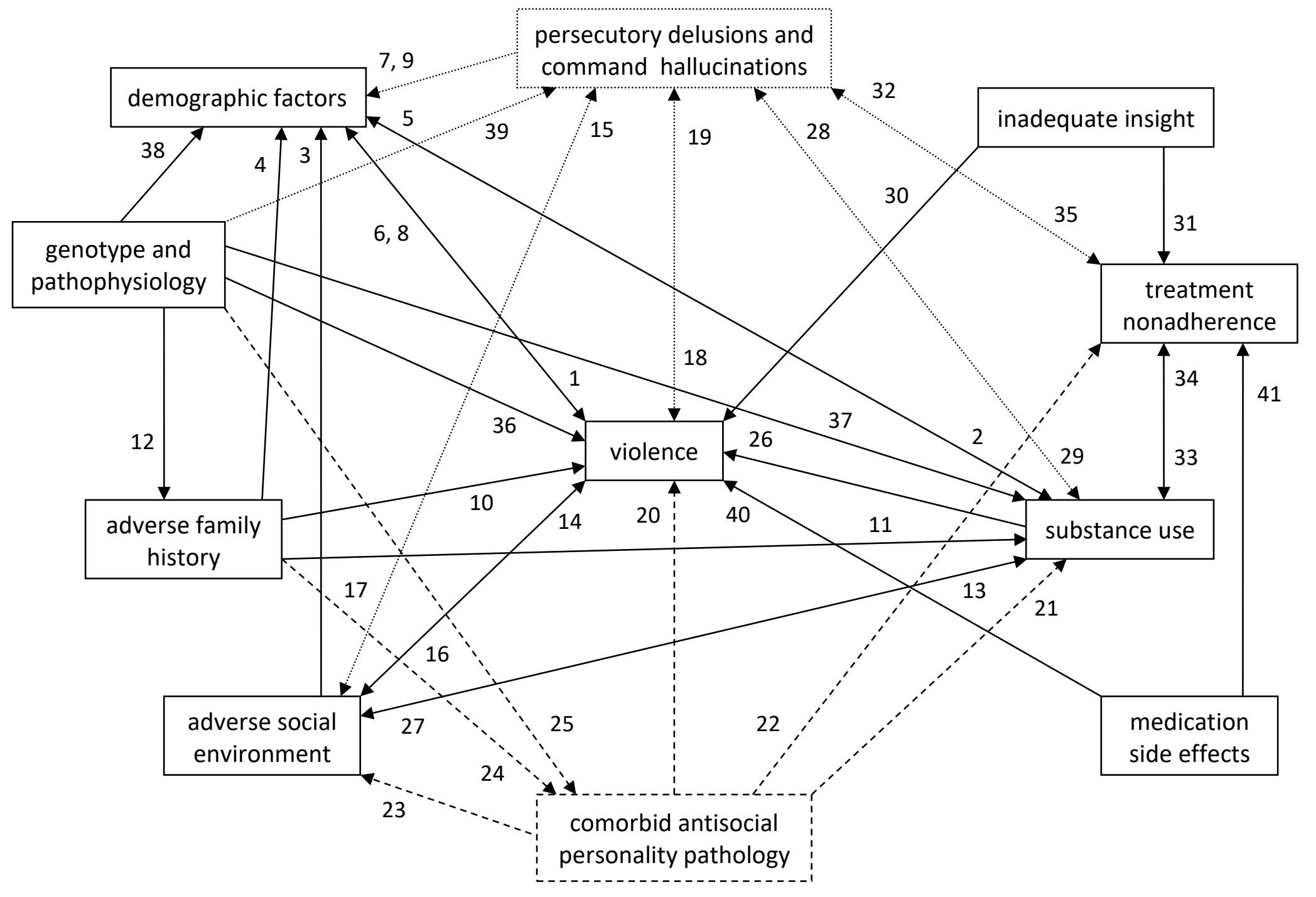


Chapter 2

\section{Discussion}

While statistically robust, the association between psychosis and violence still cannot be causally explained. This is partially due to methodological aspects of research, which include study design, sampling, operationalization of violence and psychosis, and confounding factors. Above all, the complex etiology of violent behavior in psychosis renders it difficult to establish a causal relationship between the two. Its manifestation may indeed be caused by risk factors, many of which are mutually related: demographics; social factors; persecutory delusions; command hallucinations; comorbid antisocial personality pathology; substance use; lack of insight; treatment nonadherence; and physiological factors. This study has attempted to conceptualize the causal relationship between violence and psychosis by proposing 41 potential causal pathways between these risk factors and violent behavior. To the authors' knowledge, this constitutes the most comprehensive overview of hypotheses regarding violence in psychosis to date.

However, two important limitations of the present study should be noted. First, the selection of literature used for this review is not exhaustive. Second, the methodological quality of the included studies was not systematically weighted for analysis. We have aimed to counterbalance both limitations by emphasizing the findings of previous reviews. However, the primary aim of this review is to conceptualize the associations between risk factors and violence in psychosis, not to quantify them or to evaluate the empirical evidence.

The current review holds several implications for research and practice. It stimulates research on violence in psychosis by providing a comprehensive hypothetical and interpretative framework. We also identified a number of urgent avenues of investigation. In particular, the role of persecutory delusions and command hallucinations as potential causes of violence requires further investigation. This should be done by evaluating the fluctuation of these symptoms in relation to violent behavior over time. Furthermore, studies should consider violence associated with florid psychotic symptoms as etiologically different from that associated with comorbid antisocial personality pathology. More research is also needed on the genetic and 
neurobiological underpinnings of violence in psychosis. Methodological implications arise from our review as well. More cohort studies should be conducted to determine whether the associations between risk factors and violence are causal rather than correlational. Community and institutional samples should be combined to optimize the external validity of studies. Appropriate external control subjects, such as siblings and nonviolent psychotic patients, may serve to enhance the findings' internal validity. For reliable and valid measurement, psychosis should be evaluated at symptom-level using structured interviews, while collection of data on violent behavior should combine criminal records, case notes, collateral information and selfreport measures. More uniform definitions of violence would facilitate comparison of results between studies. Finally, additional meta-analyses are needed to estimate the extent of publication bias in the field. 


\section{References}

1. American Psychiatric Association. Diagnostic and Statistical Manual of Mental Disorders (5th edition). American Psychiatric Publishing, 2013.

2. Angermeyer MC. Schizophrenia and violence. Acta Psychiatr Scand Suppl 2000; 102: 6367.

3. Bo $\mathrm{S}$, Abu-Akel A, Kongerslev $\mathrm{M}$, et al. Risk factors for violence among patients with schizophrenia. Clin Psychol Rev 2011; 31: 711-726.

4. Douglas KS, Guy LS, Hart SD. Psychosis as a risk factor for violence to others: a metaanalysis. Psychol Bull 2009; 135: 679-706.

5. Fazel S, Gulati G, Linsell L, et al. Schizophrenia and violence: systematic review and metaanalysis. PloS Med 2009; 6: e1000120.

6. Hodgins S. Violent behaviour among people with schizophrenia: a framework for investigations of causes, and effective treatment, and prevention. Philos Trans R Soc Lond B Biol Sci 2008; 363: 2505-2518.

7. Joyal CC, Dubreucq J-L, Gendron C, et al. Major mental disorders and violence: a critical update. Curr Psychiatry Rev 2007; 3: 33-50.

8. Modestin J. Criminal and violent behavior in schizophrenic patients: an overview. Psychiatry Clin Neurosci 1998; 52: 547-554.

9. Nederlof AF, Muris P, Hovens JE. The epidemiology of violent behavior in patients with a psychotic disorder: a systematic review of studies since 1980. Aggress Violent Behav 2013; 18: 83-189.

10. Volavka J. Violence in schizophrenia and bipolar disorder. Psychiatr Danub 2013; 25: 2433.

11. Walsh E, Buchanan A, Fahy T. Violence and schizophrenia: examining the evidence. $\mathrm{Br} J$ Psychiatry 2002; 180: 490-495.

12. Elbogen EB, Johnson SC. The intricate link between violence and mental disorder: results from the National Epidemiologic Survey on Alcohol and Related Conditions. Arch Gen Psychiatry 2009; 66: 152-161. 
13. Fazel S, Grann M, Carlström E, et al. Risk factors for violent crime in schizophrenia: a national cohort study of 13,806 patients. J Clin Psychiatry 2009; 70: 362-369.

14. Swanson JW, Swartz MS, Van Dorn RA, et al. A national study of violent behavior in persons with schizophrenia. Arch Gen Psychiatry 2006; 63: 490-499.

15. Witt K, Van Dorn RA, Fazel S. Risk factors for violence in psychosis: systematic review and meta-regression analysis of 110 studies. PloS One 2013; 8: e55942.

16. Cronin P, Ryan F, Coughlan M. Undertaking a literature review: a step-by-step approach. Br J Nurs 2008; 17: 38-43.

17. Marriot C, Hamilton-Giachritsis C, Harrop C. Factors promoting resilience following childhood sexual abuse: a structured, narrative review of the literature. Child Abuse Rev 2013; 23: 17-34.

18. Mann CJ. Observational research methods. Research design II: cohort, cross sectional, and case-control studies. Emerg Med J 2003; 20: 54-60.

19. Hodgins S. Major mental disorder and crime: an overview. Psychol Crime Law 1995; 2: 517.

20. Modestin J, Wuermle O. Criminality in men with major mental disorder with and without comorbid substance use. Psychiatry Clin Neurosci 2005; 59: 5-29.

21. Tiihonen J, Isohanni $M$, Räsänen $P$, et al. Specific major mental disorders and criminality: a 26-year prospective study of the 1966 Northern Finland birth cohort. Am J Psychiatry 1997; 154: 840-845.

22. Arseneault L, Cannon M, Murray R, et al. Childhood origins of violent behaviour in adults with schizophreniform disorder. Br J Psychiatry 2003; 183: 520-525.

23. Hodgins S, Hiscoke UL, Freese R. The antecedents of aggressive behavior among men with schizophrenia: a prospective investigation of patients in community treatment. Behav Sci Law 2003; 21: 523-546.

24. Moran $P$, Walsh E, Tyrer $P$, et al. Impact of comorbid personality disorder on violence in psychosis: report from the UK700 trial. Br J Psychiatry 2003; 181: 129-134.

25. Coid JW, Ullrich S, Kallis C, et al. The relationship between delusions and violence: findings from the East London first episode psychosis study. JAMA Psychiatry 2013; 70: 465-471. 
Chapter 2

26. Fresan A, Apiquian R, Nicolini H. Psychotic symptoms and the prediction of violence in schizophrenic patients. In DP French (Ed), Schizophrenic psychology: new research (239254). Nova Science Publishers, 2011.

27. Sternberg RJ, Roediger HL, Halpern DF. Critical thinking in psychology. Cambridge University Press, 2007.

28. Fazel S, Långström N, Hjern A, et al. Schizophrenia, substance abuse, and violent crime. JAMA Psychiatry 2009; 301: 2016-2023.

29. Tengström A, Hodgins S, Grann M, et al. Schizophrenia and criminal offending: the role of psychopathy and substance use disorders. Crim Justice Behav 2004; 31: 367-391.

30. Markowitz FE. Mental illness, crime, and violence: risk, context, and social control. Aggress Violent Behav 2011; 16: 36-44.

31. Mullen PE. Schizophrenia and violence: from correlations to preventive strategies. Adv Psychiatr Treat 2006; 12: 239-248.

32. Wallace C, Mullen PE, Burgess P. Criminal offending in schizophrenia over a 25-year period marked by deinstitutionalization and increasing prevalence of comorbid substance use disorders. Am J Psychiatry 2004; 161: 716-727.

33. Rogers T, Fahy T. Suicide, violence, and schizophrenia. Psychiatry 2008; 7: 482-486.

34. Gottfredson MR, Hirschi T. A general theory of crime. Stanford University Press, Stanford: 1990.

35. Erkiran $M$, Özüalan $H$, Evren $C$, et al. Substance abuse amplifies the risk for violence in schizophrenia spectrum disorder. Addict Behav 2006; 31: 1797-1805.

36. Volavka J, Citrome L. Heterogeneity of violence in schizophrenia and implications for long-term treatment. Int J Clin Pract 2008; 62: 1237-1245.

37. Hiday VA. Understanding the connection between mental illness and violence. Int J Law Psychiatry 1997; 20: 399-417.

38. Haddock G, Shaw JJ. Understanding and working with aggression, violence, and psychosis. In KT Mueser, DV Jeste (Eds), Clinical handbook of schizophrenia (398-410). The Guilford Press, 2008.

39. Volavka J, Citrome L. Pathways to aggression in schizophrenia affect results of treatment. Schizophr Bull 2011; 37: 921-929. 
40. Bjørkly S. Clinical assessment of dangerousness in psychotic patients: some risk indicators and pitfalls. Aggress Violent Behav 1997; 2: 167-178.

41. Fresán A, Apiquian R, de la Fuente-Sandoval C, et al. Violent Behavior in schizophrenic patients: relationship with clinical symptoms. Aggress Behav 2005; 31: 511-520.

42. Taylor PJ. When symptoms of psychosis drive serious violence. Soc Psychiatry Psychiatr Epidemiol 1998; 33: 47-54.

43. Taylor PJ. Psychosis and violence: stories, fears, and reality. Can J Psychiatry 2008; 53 : 647-659.

44. Bjørkly S. Psychotic symptoms and violence toward others - a literature review of some preliminary findings. Part 1: delusions. Aggress Violent Behav 2002; 7: 617-631.

45. Bjørkly S. Psychotic symptoms and violence toward others - a literature review of some preliminary findings. Part 2: hallucinations. Aggress Violent Behav 2002; 7: 605-615.

46. Cheung P, Schweitzer I, Crowley K, et al. Violence in schizophrenia: role of hallucinations and delusions. Schizophr Res 1997; 26: 181-190.

47. Fanning JR, Berman ME, Mohn RS, et al. Perceived threat mediates the relationship between psychosis proneness and aggressive behavior. Psychiatry Res 2011; 186: 210218.

48. Stompe T, Ortwein-Swoboda G, Schanda H. Schizophrenia, delusional symptoms, and violence: the threat/control-override concept reexamined. Schizophr Bull 2004; 30: 3144.

49. Bjørkly S. Empirical evidence of a relationship between insight and risk of violence in the mentally ill - a review of the literature. Aggress Violent Behav 2006; 11: 414-423.

50. Barrowcliff AL, Haddock G. The relationship between command hallucinations and factors of compliance: a critical review of the literature. J Forensic Psychiatry Psychol 2006; 17: 266-298.

51. Belli $\mathrm{H}$, Ural $\mathrm{C}$. The association between schizophrenia and violent or homicidal behaviour: the prevention and treatment of violent behaviour in these patients. West Indian Med J 2012; 61: 538-543.

52. Goethals KR, Vorstenbosch ECW, van Marle HJC. Diagnostic comorbidity in psychotic offenders and their criminal history: a review of the literature. Int J Forensic Ment Health; 7: 147-156. 
53. Hodgins S, Cree A, Alderton J, et al. From conduct disorder to severe mental illness: associations with aggressive behaviour, crime and victimization. Psychol Med 2008; 38: 975-987.

54. Hodgins S, Tiihonen J, Ross D. The consequences of conduct disorder for males who develop schizophrenia: associations with criminality, aggressive behavior, substance use, and psychiatric services. Schizophr Res 2005; 78: 323-335.

55. McGregor K, Castle D, Dolan M. Schizophrenia spectrum disorders, substance misuse, and the four-facet model of psychopathy: the relationship to violence. Schizophr Res 2012; 136: 116-121.

56. Moran P, Hodgins $\mathrm{S}$. The correlates of comorbid antisocial personality disorder in schizophrenia. Schizophr Bull 2004; 30: 791-802.

57. Tengström A, Grann M, Långström N, et al. Psychopathy (PCL-R) as a predictor of violent recidivism among criminal offenders with schizophrenia. Law Hum Behav 2000; 24: 4558.

58. Swanson JW, Van Dorn RA, Swartz MS, et al. Alternative pathways to violence in persons with schizophrenia: the role of childhood antisocial behavior problems. Law Hum Behav 2008; 32: 228-240.

59. Dumais A, Potvin S, Joyal CC, et al. Schizophrenia and serious violence: a clinical-profile analysis incorporating impulsivity and substance-use disorders. Schizophr Res 2011; 130: 234-237.

60. Soyka M. Substance misuse, psychiatric disorder and violent and disturbed behaviour. $\mathrm{Br}$ J Psychiatry 2000; 176: 345-350.

61. Gregg L, Barrowclough C, Haddock G. Reasons for increased substance use in psychosis. Clin Psychol Rev 2007; 27: 494-510.

62. Alia-Klein N, O'Rourke TM, Goldstein RZ, et al. Insight into illness and adherence to psychotropic medications are separately associated with violence severity in a forensic sample. Aggress Behav 2007; 33: 86-96.

63. Buckley PF, Hrouda DR, Friedman L, et al. Insight and its relationship to violent behavior in patients with schizophrenia. Am J Psychiatry 2004; 161: 1712-1714.

64. Calatayud GL, Sebastiá NH, García-Iturrospec EA, et al. [Relationship between insight, violence and diagnoses in psychotic patients]. Rev Psiquiatr Salud Ment 2012; 5: 43-47. 
65. Lincoln TM, Hodgins S. Is lack of insight associated with physically aggressive behavior among people with schizophrenia living in the community? J Nerv Ment Dis 2008; 196: $62-66$.

66. Freeman D, Garety P. Helping patients with paranoid and suspicious thoughts: a cognitive-behavioural approach. Adv Psychiatr Treat 2006; 12: 404-441.

67. Swartz MS, Swanson JE, Hiday VA, et al. Violence and severe mental illness: the effects of substance abuse and nonadherence to medication. Am J Psychiatry 1998; 155: 226-231.

68. Cheung P, Schweitzer I. Correlates of aggressive behaviour in schizophrenia: an overview. Aust N Z J Psychiatry 1998; 32: 400-409.

69. Hoptman MJ, Antonius D. Neuroimaging correlates of aggression in schizophrenia: an update. Curr Opin Psychiatry 2011; 24: 100-106.

70. Naudts K, Hodgins S. Neurobiological correlates of violent behavior among persons with schizophrenia. Schizophr Bull 2006; 32: 562-572.

71. Soyka M. Neurobiology of aggression and violence in schizophrenia. Schizophr Bull 2011; 37: 913-920.

72. Seo $D$, Patrick $C J$. Role of serotonin and dopamine system interactions in the neurobiology of impulsive aggression and its comorbidity with other clinical disorders. Aggress Violent Behav 2008; 13: 383-395.

73. Weiss EM. Neuroimaging and neurocognitive correlates of aggression and violence in schizophrenia. Scientifica 2012: 158646. 



\section{Chapter 3}

Use of illicit substances and violent behavior in psychotic disorders: two nationwide casecontrol studies and meta-analyses

Lamsma J

Cahn W

Fazel S

GROUP and NEDEN investigators

Psychol Med 2020; 50: 2028-2033. 
Chapter 3

\section{Abstract}

\section{Background}

Substance use disorder explains much of the excess risk of violent behavior in psychotic disorders. However, it is unclear to what extent the pharmacological properties and subthreshold use of illicit substances are associated with violence.

\section{Methods}

Individuals with psychotic disorders were recruited for two nationwide projects: GROUP ( $N=$ $871)$ in the Netherlands and NEDEN $(N=921)$ in the United Kingdom. Substance use and violent behavior were assessed with standardized instruments and multiple sources of information. First, we used logistic regression models to estimate the associations of daily and nondaily use with violence for cannabis, stimulants, depressants and hallucinogens in the GROUP and NEDEN samples separately. Adjustments were made for age, sex and educational level. We then combined the results in random-effects meta-analyses.

\section{Results}

Daily use, compared with nondaily or no use, and nondaily use, compared with no use, increased the pooled odds of violence for all substance categories. The increases were significant for daily use of cannabis (pooled odds ratio [pOR] 1.6, 95\% confidence interval [CI] 1.22.0), stimulants (pOR 2.8, 95\% $\mathrm{Cl} 1.7-4.5$ ) and depressants (pOR 2.2, 95\% $\mathrm{Cl} 1.1-4.5$ ), and nondaily use of stimulants (pOR 1.6, 95\% Cl 1.2-2.0) and hallucinogens (pOR 1.5, 95\% Cl 1.1-2.1). Daily use of hallucinogens, which could only be analyzed in the NEDEN sample, significantly increased the risk of violence (adjusted odds ratio $3.3,95 \% \mathrm{Cl} 1.2-9.3$ ).

\section{Conclusion}

Strategies to prevent violent behavior in psychotic disorders should target any substance use. 


\section{Introduction}

Much of the excess risk of violent behavior in psychotic disorders can be explained by substance use disorder (SUD) ${ }^{1}$. In a meta-analysis of 16 studies with a total of 5,365 cases, SUD more than doubled the odds of violence (odds ratio $[\mathrm{OR}] 2.2,95 \%$ confidence interval $[\mathrm{Cl}] 1.6$ 2.9) $)^{2}$.

However, it is unclear to what extent different categories of illicit substances, as defined by their psychopharmacological effects, are related to violent behavior. Another uncertainty is whether subthreshold use, as opposed to SUD, is a risk factor for violence. These questions may be clinically relevant, as the psychopharmacological properties of substances modify violence risk in the general population ${ }^{3}$ and people with psychotic disorders are highly sensitive to the harmful effects of substances ${ }^{4}$. The few studies of cannabis ${ }^{5-7}$ and stimulants ${ }^{8-10}$ have produced conflicting results. Moreover, these studies have been limited by small samples of inpatients and proxy measures of violent behavior (e.g. hostility, aggression). A recent metaanalysis of 12 studies involving 3,873 subjects with severe mental illness - but not psychotic disorders specifically - reported a significant association between cannabis use and violence (pooled odds ratio [pOR] 3.0, 95\% Cl 2.0-4.5) ${ }^{11}$. To our knowledge, there have been no studies of depressants (besides alcohol) or hallucinogens.

To address the limitations of previous studies, we have investigated the associations of daily and nondaily use with violent behavior for cannabis, stimulants, depressants and hallucinogens in two nationwide samples of individuals with psychotic disorders.

\section{Methods}

We used baseline data from two research projects: Genetic Risk and Outcome of Psychosis $(\text { GROUP) })^{12}$ and National Evaluation of the Development and Impact of Early Intervention Services (NEDEN) ${ }^{13}$. 
Chapter 3

Setting and participants

GROUP

GROUP is conducted by four university medical centers (i.e. Amsterdam Medical Center, Maastricht University Medical Center+, University Medical Center Groningen, University Medical Center Utrecht) and affiliated mental health centers $(k=36)$ in the Netherlands. These centers are located in geographically representative areas of the country and provide access to treatment in a variety of settings (e.g. psychiatric hospitals, outpatient clinics) to approximately $75 \%$ of the population. Recruitment took place in 2004 . To be eligible for participation, patients had to (i) be aged between 16 and 50, (ii) have a good command of the Dutch language and (iii) meet Diagnostic and Statistical Manual of Mental Disorders, Fourth Edition, Text Revision (DSM-IV-TR) ${ }^{14}$ criteria for schizophrenia or another psychotic disorder. In accordance with standard local practice, DSM-IV-TR diagnoses were made with the Comprehensive Assessment of Symptoms and History ${ }^{15}$ or Schedules for Clinical Assessment for Neuropsychiatry ${ }^{16}$.

\section{NEDEN}

All individuals enrolled into Early Intervention Services (EIS) at five sites across England (i.e. Birmingham, Cornwall, Cambridge, Norwich, Lancashire) between 2005 and 2009 were invited to participate. Sites were chosen to reflect urban and rural differences. The Department of Health and Social Care requires that people receiving EIS are between 14 and 35 years old and present with a first episode of psychosis. No additional inclusion criteria were set. The Operationalized Criteria System ${ }^{17}$ was used to determine International Statistical Classification of Diseases and Related Health Problems, Tenth Edition (ICD-10) ${ }^{18}$ diagnoses of mental disorders. 


\section{Measures}

Information about the instruments' psychometric properties can be found in the relevant publications for GROUP ${ }^{12}$ and NEDEN ${ }^{13}$. Unless otherwise specified, the reference period was the lifetime.

GROUP

\section{Substance use}

The Substance Abuse Module of the Composite International Diagnostic Interview (CIDISAM) ${ }^{19}$ was used to measure the frequency (i.e. daily use, nondaily use, no use) and severity (i.e. problematic use, nonproblematic use, no use) of substance use. The CIDI-SAM distinguishes between the following categories of substances: (i) alcohol; (ii) cannabis; (iii) cocaine; (iv) stimulants (e.g. amphetamine, khat); (v) sedatives (e.g. pentobarbital, diazepam); (vi) opiates (e.g. heroin, codeine); (vii) inhalants (e.g. toluene, butane); (viii) phencyclidine (PCP); (ix) psychedelics [e.g. lysergic acid diethylamide (LSD), mescaline]; and (x) other substances [e.g. amyl nitrite, 3,4-methylenedioxymethamphetamine (MDMA)]. Based on considerations of statistical power and similarities in psychopharmacological effects, we combined cocaine and stimulants as 'stimulants', sedatives, opiates and inhalants as 'depressants' and PCP and psychedelics as 'hallucinogens' ${ }^{20}$. Cannabis, which has stimulant, depressive and hallucinogenic properties, was treated separately owing to the high prevalence of its use. We defined problematic alcohol use as an average intake of more than 18 standard drinks per week for men and more than 12 standard drinks per week for women during a minimum period of 2 weeks in the past year or 4 weeks at any other point in the past. These cutoffs reflect the median of several national guidelines and a consistent 1.5:1 male to female consumption ratio ${ }^{21}$. For other substances, problematic use corresponded to a DSM-IV-TR diagnosis of abuse or dependence. 
Chapter 3

\section{Violent behavior}

Violent behavior was established with the Life Chart Schedule (LCS) ${ }^{22}$. Designed to record the development of symptoms, health care consumption and social functioning in schizophrenia patients, the LCS contains the following question regarding violence: 'Did the patient physically attack or abuse someone else?' The LCS was filled out based on review of clinical case notes and interviews with the patient and, if possible, one or both parents.

\section{NEDEN}

\section{Substance use}

A purposely designed questionnaire was used to assess substance use. For 15 substance categories, patients were asked whether they had used them: (i) almost every day; (ii) 1 to 3 times per week; (iii) less than once per week; (iv) 3 times or less; or (v) never. For the sake of consistency, we combined frequency categories ii, iii and iv as 'nondaily use' and refer to 'almost every day' as 'daily'. The substance categories were rearranged as follows: (i) cannabis; (ii) stimulants (i.e. cocaine, amphetamine, khat); (iii) depressants (i.e. opiates, $y$-hydroxybutyric acid, barbiturates, benzodiazepines, solvents, 'poppers'); (iv) hallucinogens (i.e. LSD, psilocybin, ketamine); and (v) other substances (i.e. MDMA, 'other') ${ }^{20}$.

\section{Violent behavior}

Violent behavior was ascertained from patient and clinician interviews using the Adverse Outcomes Questionnaire (AOQ). In the $A O Q$, a shortened version of the questionnaire used in the MacArthur Violence Risk Assessment Study ${ }^{23}$, violence is operationalized to encompass: (i) battery that resulted in physical injury; (ii) sexual assault; (iii) assault involving the use of a 
weapon; (iv) threats made with a weapon in hand; and (v) battery that did not result in physical injury. The $A O Q$ referred to the past 12 months.

\section{Analyses}

First, we used logistic regression models to estimate the associations of daily and nondaily use with violent behavior for cannabis, stimulants, depressants and hallucinogens in the GROUP and NEDEN samples separately. Three comparisons were made: (i) daily use $v$. nondaily or no use; (ii) daily use v. no use; and (iii) nondaily use v. no use. For theoretical reasons, we included the confounders age, sex and educational level ${ }^{24}$. Educational level, indicating whether a patient had completed secondary school, served as a proxy for socioeconomic status $^{25}$. We only analyzed complete cases. Depending on the scale of measurement, complete and incomplete cases were compared on each model variable with the $\chi^{2}$-test (dichotomous) or $t$-test (continuous). To improve validity, we required models with at least 5 observations per cell in the $2 \times 2$ table of the exposure and outcome of interest.

We then combined the results for the GROUP and NEDEN samples in random-effects metaanalyses. The $I^{2}$ statistic was used as a measure of heterogeneity. Values of $25 \%, 50 \%$ and $75 \%$ denoted low, moderate and high levels of heterogeneity, respectively ${ }^{26}$.

For sensitivity analyses, we examined severity of use. Alcohol, which has consistently been found to increase violence risk in people with psychotic disorders ${ }^{2}$, was used as a positive control.

The level of statistical significance was set at 5\%. Analyses were carried out in STATA 12.1 . 
Chapter 3

Results

Of the 1,013 patients in the GROUP sample, $871(86 \%)$ had data on all model variables and were thus included in the analyses. The corresponding numbers in the NEDEN sample were 1,027 and 921 (90\%), respectively. Complete cases differed significantly from incomplete cases on age $(t[1,011]=3.31, p=.001)$ in the GROUP sample (Table S1) and educational level $\left(\chi^{2}[1]=3.89, p=.049\right)$ in the NEDEN sample (Table S2).

Demographic and clinical characteristics of patients

GROUP

Demographic and clinical characteristics of the patients $(N=871)$ are presented in Table 1 . The mean age was 27.3 years $(S D=7.1)$. Most patients were male $(n=673,77 \%)$ and had received a diagnosis of schizophrenia ( $n=602,69 \%)$. Use of illicit substances was reported by 602 (69\%) patients. About one if five patients had been violent ( $n=179,21 \%)$.

\section{NEDEN}

The patients $(N=921)$ had a mean age of 22.8 years $(S D=4.8)$ and were predominantly male ( $n=639,69 \%$ ). (Table 1). The most common diagnosis was schizophrenia ( $n=478,47 \%$ ). Almost two thirds of the patients had used illicit substances ( $n=589,64 \%)$. The prevalence of violent behavior was $22 \%(n=204)$. 
Table 1. Demographic and clinical characteristics of patients in the GROUP $(N=871)$ and NEDEN $(N=921)$ samples.

\begin{tabular}{lcc}
\hline & GROUP & NEDEN \\
\hline Demographic characteristics & & \\
Age, mean (SD) in years & $27.3(7.1)$ & $22.8(4.8)$ \\
Male & $673(77)$ & $639(69)$ \\
Caucasian & $679(79)$ & $679(74)$ \\
Completed secondary school & $753(86)$ & $811(88)$ \\
& & \\
Clinical characteristics & & \\
Psychiatric diagnosis ${ }^{\text {a }}$ & & \\
Schizophrenia & $602(69)$ & $478(47)$ \\
Schizoaffective disorder & $106(12)$ & $70(7)$ \\
Psychotic disorder NOS & $74(8)$ & $190(19)$ \\
Other & $89(10)$ & $289(28)$ \\
Age of onset, mean (SD) in years & $23.1(6.5)$ & $21.3(4.9)$ \\
Use of illicit substances & & \\
Daily & $430(49)$ & $314(34)$ \\
Cannabis & $411(48)$ & $285(31)$ \\
Stimulants & $68(8)$ & $55(6)$ \\
Depressants & $51(6)$ & $28(3)$ \\
Hallucinogens & $6(1)$ & $15(2)$ \\
Other & $23(3)$ & $21(2)$ \\
Nondaily & $427(49)$ & $480(52)$ \\
Cannabis & $170(20)$ & $279(30)$ \\
Stimulants & $198(24)$ & $245(27)$ \\
Depressants & $62(8)$ & $89(10)$ \\
Hallucinogens & $165(20)$ & $161(17)$ \\
Other & $212(26)$ & $230(25)$ \\
Violent behavior & $179(21)$ & $204(22)$ \\
\hline SD, standard deviation; NOS, not otherwise specified. & \\
Data are $n$ (\%), unless otherwise stated. & & \\
a Psychiatric diagnoses were only available for the full NEDEN sample $(N=1,072)$
\end{tabular}


Chapter 3

Primary analyses

GROUP

Daily use, compared with nondaily or no use, and nondaily use increased the adjusted odds of violent behavior for all substance categories (Table 2). The increases were significant for daily use of stimulants (adjusted odds ratio [aOR] 2.2, 95\% Cl 1.3-3.8) and nondaily use of hallucinogens (aOR 1.8, 95\% Cl 1.2-2.7).

NEDEN

When comparing daily use with nondaily or no use, cannabis (aOR 1.8,95\% $\mathrm{Cl} 1.3-2.5$ ), stimulants (aOR 3.6, 95\% $\mathrm{Cl}$ 2.1-6.4), depressants (aOR 3.3, 95\% Cl 1.5-7.2) and hallucinogens (aOR $3.3,95 \% \mathrm{Cl} 1.2-9.3$ ) significantly increased the adjusted odds of violence (Table 2). Nondaily use increased the aORs for these substance categories as well, with that for stimulants reaching statistical significance (aOR 1.6, 95\% Cl 1.2-2.3).

Meta-analyses

Pooled across the GROUP and NEDEN samples, daily use of cannabis (pOR 1.6, 95\% Cl 1.2-2.0), stimulants (pOR 2.8, 95\% Cl 1.7-4.5) and depressants (pOR 2.2, 95\% Cl 1.1-4.5) significantly increased the odds of violence compared with nondaily or no use (Table 3). The same was found for nondaily use of stimulants (pOR 1.6, 95\% $\mathrm{Cl} 1.2-2.0$ ) and hallucinogens (pOR 1.5, 95\% $\mathrm{Cl}$ 1.1-2.1). Moderate heterogeneity was present for daily use of depressants in both comparisons $\left(I^{2}=46 \%, 50 \%\right)$. Otherwise, heterogeneity was low $\left(I^{2} \leq 36 \%\right)$. 
Table 2. Prevalence and risk of violent behavior by different categories of illicit substances and frequency of their use in the GROUP $(N=871)$ and NEDEN $(N=921)$ samples.

\begin{tabular}{|c|c|c|c|c|c|c|}
\hline \multirow[b]{2}{*}{ Substance category } & \multicolumn{3}{|c|}{$n(\%)$} & \multicolumn{3}{|c|}{$\mathrm{aOR}(95 \% \mathrm{Cl})^{\mathrm{a}}$} \\
\hline & DU & NDU & NU & DU vs NDU or NU & DU vs NU & NDU vs NU \\
\hline \multicolumn{7}{|l|}{ GROUP } \\
\hline Cannabis & $99(24)$ & $36(21)$ & $44(15)$ & 1.4 (1.0-1.9) & $1.5(1.0-2.3)$ & $1.3(0.8-2.2)$ \\
\hline Stimulants & $25(37)$ & $48(24)$ & $98(17)$ & $2.2(1.3-3.8)$ & $2.6(1.5-4.5)$ & $1.5(1.0-2.2)$ \\
\hline Depressants & $15(29)$ & $18(29)$ & $134(19)$ & $1.6(0.9-3.1)$ & $1.7(0.9-3.3)$ & $1.7(0.9-3.0)$ \\
\hline Hallucinogens & $3(50)$ & $46(28)$ & $113(17)$ & - & - & $1.8(1.2-2.7)$ \\
\hline Other & $8(35)$ & $57(27)$ & $100(17)$ & $1.9(0.8-4.6)$ & $2.4(1.0-5.8)$ & $1.7(1.2-2.6)$ \\
\hline \multicolumn{7}{|l|}{ NEDEN } \\
\hline Cannabis & $88(30)$ & $60(21)$ & $61(17)$ & $1.8(1.3-2.5)$ & $2.0(1.3-2.9)$ & $1.2(0.8-1.8)$ \\
\hline Stimulants & $26(46)$ & $71(28)$ & $112(17)$ & $3.6(2.1-6.4)$ & $4.3(2.4-7.8)$ & $1.6(1.2-2.3)$ \\
\hline Depressants & $13(43)$ & $23(26)$ & $173(21)$ & $3.3(1.5-7.2)$ & $3.4(1.6-7.3)$ & $1.2(0.7-2.0)$ \\
\hline Hallucinogens & $7(47)$ & $44(27)$ & $158(21)$ & $3.3(1.2-9.3)$ & $3.4(1.2-9.6)$ & $1.3(0.9-1.9)$ \\
\hline Other & $10(45)$ & $65(27)$ & $134(19)$ & $3.4(1.4-8.2)$ & $3.9(1.6-9.3)$ & $1.5(1.0-2.1)$ \\
\hline
\end{tabular}

aOR, adjusted odds ratio; $\mathrm{Cl}$, confidence interval; $\mathrm{DU}$, daily use; $\mathrm{NDU}$, nondaily use; $\mathrm{NU}$, no use

Due to missing data, the total number of patients varies by substance category.

${ }^{\text {a }}$ Adjusted for age, sex and educational level. 
Chapter 3

Table 3. Risk of violent behavior by different categories of illicit substances and frequency of their use, pooled across the GROUP $(N=871)$ and NEDEN $(N=921)$ samples.

\begin{tabular}{lccc}
\hline & \multicolumn{3}{c}{ pOR $(95 \% \mathrm{Cl})$} \\
\cline { 2 - 4 } Substance category & DU vs NDU or NU & DU vs NU & NDU vs NU \\
\hline Cannabis & $1.6(1.2-2.0)$ & $1.7(1.3-2.3)$ & $1.2(0.9-1.7)$ \\
Stimulants & $2.8(1.7-4.5)$ & $3.3(2.0-5.4)$ & $1.6(1.2-2.0)$ \\
Depressants & $2.2(1.1-4.5)$ & $2.3(1.2-4.6)$ & $1.4(0.9-2.1)$ \\
Hallucinogens & - & - & $1.5(1.1-2.1)$ \\
Other & $2.5(1.4-4.7)$ & $3.1(1.6-5.7)$ & $1.6(1.2-2.1)$ \\
\hline
\end{tabular}

pOR, pooled odds ratio; $\mathrm{Cl}$, confidence interval; $\mathrm{DU}$, daily use; NDU, nondaily use; $\mathrm{NU}$, no use. 
Sensitivity analyses

We observed no material differences in results after repeating the analyses in the GROUP sample with severity of use. As expected, alcohol increased the adjusted odds of violence (Table S3).

\section{Discussion}

In two nationwide samples totaling 1,792 patients, we investigated associations between frequency of use and violence for different categories of illicit substances. Overall, daily and nondaily use of cannabis, stimulants, depressants and hallucinogens were found to increase violence risk.

There are at least four ways in which substance use may bring about violent behavior in psychotic disorders. First, psychopharmacological effects of intoxication with or withdrawal from substances (e.g. disinhibition, intensification of negative emotions) may lower the threshold for violence ${ }^{27}$. Substance use may also induce or exacerbate positive symptoms (e.g. delusions, hallucinations $)^{28}$, which are risk factors for violent behavior ${ }^{2}$. This may be particularly relevant for cannabis and hallucinogens. The former has been found to increase the risk of developing a psychotic disorder ${ }^{29}$, and the latter - with the possible exception of PCP - are thought not to increase violence risk in the general population ${ }^{3}$. Second, substance use may interfere with treatment. Individuals with problematic substance use are less likely to seek and adhere to treatment than those without these substance problems ${ }^{28}$. At the same time, substances may be used in an attempt to alleviate psychotic symptoms or unpleasant side effects of antipsychotics ${ }^{4}$. Self-medication increases the likelihood of avoidance or discontinuation of treatment and vice versa ${ }^{30}$. Substances may also reduce the therapeutic activity of antipsychotics ${ }^{31}$. In the absence of effective treatment, positive symptoms may persist or worsen. Third, violence may occur during the commission of crimes to gain access to sub- 
stances or the money to buy them ${ }^{32}$. Finally, users may become involved in illegal drug markets where violent behavior is commonplace ${ }^{33}$. Other explanations for the findings are confounding or mediation by biological (e.g. genetics, neurobiological abnormalities), psychological (e.g. cognitive impairment, personality pathology) or environmental (e.g. childhood maltreatment, erosion of social support) risk factors ${ }^{24}$.

As far as we know, this is the largest study to investigate the relationship between use of illicit substances and violent behavior in psychotic disorders. It has several strengths. First, the samples were drawn from diverse geographic areas and care settings. Sampling was also independent of the exposures and outcome of interest. This enhanced the generalizability of the results. Second, the use of multiple data sources increased the sensitivity of the LCS and AOQ as measures of violence. Finally, the findings for alcohol (as a positive control) were in the expected direction, supporting the validity of the design. However, there are several limitations. First, causality cannot be inferred, as the temporal relationship between substance use and violent behavior was not known and we did not control for other confounders besides age, sex and educational level. Second, we included individuals who had used substances belonging to different categories, which may have biased risk estimates. Exclusion would have made cell counts too low for meaningful analyses of most substance categories. Third, daily use was a proxy measure of SUD. However, similar results were obtained for DSM-IV-TR diagnoses of abuse and dependence in the GROUP sample. Fourth, the definition and reference period for violence varied between GROUP and NEDEN. The more stringent definition and shorter reference period may explain why aORs were slightly higher in the NEDEN sample. Fifth, missing data may have limited the validity of the results. Sixth, the results of the metaanalyses should be treated with some caution: the estimation of the between-study variance, which is used in the calculation of the pooled effect size and its confidence interval, may be inaccurate when the number of studies is small ${ }^{34}$. Seventh, we were unable to analyze PCP separately. Either too few patients had used PCP (GROUP) or no specific information was recorded for PCP (NEDEN). Finally, there has been a large increase in the use of novel psychoactive substances (NPS) in the years following data collection ${ }^{35}$. NPS are synthetic compounds designed to mimic the psychopharmacological effects of traditional substances ${ }^{36}$. Therefore, we hypothesize that NPS increase violence risk. 
A clinical implication of the findings is that violence risk assessment in psychotic disorders should target any substance use. For structured instruments, it should be determined whether items for SUD and subthreshold use of different substance categories have incremental validity over a single item for SUD. The findings also suggest that interventions, which currently focus on SUD, may assist in the prevention of violent behavior in patients with subthreshold use. To clarify causal mechanisms, we recommend that studies further isolate the psychopharmacological effects of substances, use prospective designs and test for additional confounders and mediators. 


\section{References}

1. Fazel S, Gulati G, Linsell L, et al. Schizophrenia and violence: systematic review and metaanalysis. PLoS Med 2009; 6: e1000120.

2. Witt K, Van Dorn RA, Fazel S. Risk factors for violence in psychosis: systematic review and meta-regression analysis of 110 studies. PLoS One 2013; 8: e55942.

3. Tomlinson MF, Brown M, Hoaken PN. Recreational drug use and human aggressive behavior: a comprehensive review since 2003. Aggress Violent Behav 2016; 27: 9-29.

4. Gregg L, Barrowclough C, Haddock G. Reasons for increased substance use in psychosis. Clin Psychol Rev 2007; 27: 494-510.

5. Koen L, Kinnear CJ, Corfield VA, et al. Violence in male patients with schizophrenia: risk markers in a South African population. Aust N Z J Psychiatry 2004; 38: 254-259.

6. Moulin V, Baumann P, Gholamrezaee M, et al. Cannabis, a significant risk factor for violent behavior in the early phase psychosis. Two patterns of interaction of factors increase the risk of violent behavior: cannabis use disorder and impulsivity; cannabis use disorder, lack of insight and treatment adherence. Front Psychiatry 2018; 9: 294.

7. Oluwoye O, Monroe-DeVita M, Burduli E, et al. Impact of tobacco, alcohol and cannabis use on treatment outcomes among patients experiencing first episode psychosis: data from the national RAISE-ETP study. Early Interv Psychiatry 2019; 13: 142-146.

8. Bell $M$, Greig $T$, Gill $P$, et al. Work rehabilitation and patterns of substance use among persons with schizophrenia. Psychiatr Serv 2002; 53: 63-69.

9. Harris AW, Large MM, Redoblado-Hodge $A$, et al. Clinical and cognitive associations with aggression in the first episode of psychosis. Aust N Z J Psychiatry 2010; 44: 85-93.

10. Miles $\mathrm{H}$, Johnson $\mathrm{S}$, Amponsah-Afuwape $\mathrm{S}$, et al. Characteristics of subgroups of individuals with psychotic illness and a comorbid substance use disorder. Psychiatr Serv 2003; 54: 554-561.

11. Dellazizzo L, Potvin S, Beaudoin M, et al. Cannabis use and violence in patients with severe mental illnesses: a meta-analytical investigation. Psychiatry Res 2019; 274: 42-48.

12. Korver N, Quee PJ, Boos HB, et al. Genetic Risk and Outcome of Psychosis (GROUP), a multi-site longitudinal cohort study focused on gene-environment interaction: 
objectives, sample characteristics, recruitment and assessment methods. Int J Methods Psychiatr Res 2012; 21: 205-221.

13. Birchwood M, Lester H, McCarthy L, et al. The UK national evaluation of the development and impact of Early Intervention Services (the National EDEN studies): study rationale, design and baseline characteristics. Early Interv Psychiatry 2014; 8: 59-67.

14. American Psychiatric Association. Diagnostic and Statistical Manual of Mental Disorders (4th edition, text revision). Author, 2000.

15. Andreasen NC, Flaum M, Arndt S. The Comprehensive Assessment of Symptoms and History (CASH). An instrument for assessing diagnosis and psychopathology. Arch Gen Psychiatry 1992; 49: 615-623.

16. Wing JK, Babor T, Brugha $T$, et al. SCAN: Schedules for Clinical Assessment in Neuropsychiatry. Arch Gen Psychiatry 1990; 47: 589-593.

17. McGuffin P, Farmer A, Harvey I. A polydiagnostic application of operational criteria in studies of psychotic illness. Arch Gen Psychiatry 1991; 48: 764-770.

18. World Health Organization. The ICD-10 classification of mental and behavioural disorders: clinical descriptions and diagnostic guidelines. WHO, 1992.

19. World Health Organization. Composite International Diagnostic Interview. WHO, 1990.

20. Hill SL, Thomas SH. Drugs of abuse. Medicine 2016; 44: 160-169.

21. Furtwaengler NA, de Visser RO. Lack of international consensus in low-risk drinking guidelines. Drug Alcohol Rev 2013; 32: 11-18.

22. Susser E, Finnerty M, Mojtabai R, et al. Reliability of the life chart schedule for assessment of the long-term course of schizophrenia. Schizophr Res 2000; 42: 67-77.

23. Steadman HJ, Mulvey EP, Monahan J, et al. Violence by people discharged from acute psychiatric inpatient facilities and by others in the same neighborhoods. Arch Gen Psychiatry 1998; 55: 393-401.

24. Lamsma J, Harte JM. Violence in psychosis: conceptualizing its causal relationship with risk factors. Aggress Violent Behav 2015; 24: 75-82.

25. Maksimović MŽ, Vlajinac HD, Radak ĐJ, et al. Association of socioeconomic status measured by education and risk factors for carotid atherosclerosis: cross-sectional study. Croat Med J 2018; 49, 824-831. 
26. Higgins J, Thompson S, Deeks J, et al. Measuring inconsistency in meta-analyses. BMJ 2003; 327: 557-560.

27. Kuhns JB, Clodfelter TA. Illicit drug-related psychopharmacological violence: the current understanding within a causal context. Aggress Violent Behav 2009; 14: 69-78.

28. Winklbaur B, Ebner N, Sachs G, et al. Substance abuse in patients with schizophrenia. Dialogues Clin Neurosci 2006; 8: 37-43.

29. Di Forti M, Quattrone D, Freeman TP, et al. The contribution of cannabis use to variation in the incidence of psychotic disorder across Europe (EU-GEI): a multicentre case-control study. Lancet Psychiatry 2019; 6: 427-436.

30. Swartz MS, Swanson JE, Hiday VA, et al. Violence and severe mental illness: the effects of substance abuse and nonadherence to medication. Am J Psychiatry 1998; 155: 226-231.

31. Lindsey WT, Stewart D, Childress D. Drug interactions between common illicit drugs and prescription therapies. Am J Drug Alcohol Abuse 2012; 38: 334-343.

32. McGinty EE, Choksy S, Wintemute GJ. The relationship between controlled substances and violence. Epidemiol Rev 2016; 38: 5-31.

33. Hodgins S. Violent behaviour among people with schizophrenia: a framework for investigations of causes, and effective treatment, and prevention. Philos Trans R Soc Lond B Biol Sci 2008; 363: 2505-2518.

34. Borenstein M, Hedges LV, Higgins JP, et al. Introduction to meta-analysis. Wiley, 2009.

35. Tracy DK, Wood DM, Baumeister D. Novel psychoactive substances: types, mechanisms of action, and effects. BMJ 2017; 356: i6848.

36. Miliano C, Serpelloni G, Rimondo C, et al. Neuropharmacology of new psychoactive substances (NPS): focus on the rewarding and reinforcing properties of cannabimimetics and amphetamine-like stimulants. Front Neurosci 2016; 10: 153. 
Use of illicit substances and violent behavior in psychotic disorders

Supplementary material 
Table S1. Comparisons on model variables between complete and incomplete cases in the GROUP sample $(N=1,013)$.

\begin{tabular}{lcccc}
\hline Model variable & CC $(n=871)$ & IC $(n=142)$ & Test statistic $(d f)$ & $p$ \\
\hline Age, mean (SD) in years & $27.3(7.1)$ & $25.2(6.3)$ & $t(1,011)=3.31$ & .001 \\
Male & $673(77)$ & $117(82)$ & $\chi^{2}(1)=1.87$ & $\mathrm{~ns}$ \\
Completed secondary school $^{\mathrm{a}}$ & $753(86)$ & $106(80)$ & $\chi^{2}(1)=3.53$ & $\mathrm{~ns}$ \\
Use of illicit substances $_{\text {Violent behavior }}^{602(69)}$ & $89(75)$ & $\chi^{2}(1)=1.60$ & $\mathrm{~ns}$ \\
\hline
\end{tabular}

$\mathrm{CC}$, complete cases; IC, incomplete cases; $d f$, degrees of freedom; SD, standard deviation; ns, nonsignificant. Data are $n(\%)$, unless otherwise stated. Due to missing data, the number of incomplete cases varies by model variable. 
Table S2. Comparisons on model variables between complete and incomplete cases in the NEDEN sample $(N=1,027)$.

\begin{tabular}{lcccc}
\hline Model variable & CC $(n=921)$ & IC $(n=106)$ & Test statistic $(d f)$ & $p$ \\
\hline Age, mean (SD) in years & $22.8(4.8)$ & $22.1(5.3)$ & $t(1,008)=1.35$ & $\mathrm{~ns}$ \\
Male & $639(69)$ & $70(66)$ & $\chi^{2}(1)=0.50$ & $\mathrm{~ns}$ \\
Completed secondary school & $811(88)$ & $61(80)$ & $\chi^{2}(1)=3.89$ & .049 \\
Use of illicit substances & $589(64)$ & $46(67)$ & $\chi^{2}(1)=0.21$ & $\mathrm{~ns}$ \\
Violent behavior & $204(22)$ & $15(23)$ & $\chi^{2}(1)=0.03$ & $\mathrm{~ns}$ \\
\hline
\end{tabular}

$\mathrm{CC}$, complete cases; IC, incomplete cases; $d f$, degrees of freedom; SD, standard deviation; ns, nonsignificant. Data are $\mathrm{n}(\%)$, unless otherwise stated. Due to missing data, the number of incomplete cases varies by model variable. 
Table S3. Prevalence and risk of violent behavior by severity of use of alcohol ${ }^{\mathrm{a}}$ and different categories of illicit substances in the GROUP sample $(N=871)$.

\begin{tabular}{lcccccccc}
\hline & \multicolumn{3}{c}{$n(\%)$} & & \multicolumn{3}{c}{ aOR (95\% CI) } \\
\cline { 2 - 5 } \cline { 6 - 8 } Substance category & PU & NPU & NU & & PU vs NPU or NU & PU vs NU & NPU vs NU \\
\hline Alcohol & $83(24)$ & $74(19)$ & $21(16)$ & & $1.5(1.1-2.1)$ & $1.8(1.0-3.1)$ & $1.3(0.7-2.2)$ \\
Cannabis & $90(24)$ & $45(21)$ & $44(15)$ & & $1.3(0.9-1.9)$ & $1.5(1.0-2.4)$ & $1.4(0.8-2.2)$ \\
Stimulants & $43(30)$ & $31(23)$ & $98(17)$ & & $1.8(1.2-2.7)$ & $2.0(1.3-3.0)$ & $1.4(0.9-2.3)$ \\
Depressants & $15(33)$ & $18(24)$ & $134(19)$ & & $1.9(1.0-3.7)$ & $2.0(1.0-3.9)$ & $1.3(0.7-2.4)$ \\
Hallucinogens & $12(27)$ & $41(30)$ & $113(17)$ & & $1.3(0.7-2.7)$ & $1.6(0.8-3.2)$ & $2.0(1.3-3.1)$ \\
Other & $28(29)$ & $42(28)$ & $100(17)$ & & $1.5(0.9-2.4)$ & $1.9(1.1-3.1)$ & $1.9(1.2-2.9)$ \\
\hline
\end{tabular}

aOR, adjusted odds ratio; $\mathrm{Cl}$, confidence interval; PU, problematic use; NPU, nonproblematic use; NU, no use.

Due to missing data, the total number of patients varies by substance category.

a Alcohol was used as a positive control.

${ }^{\mathrm{b}}$ Adjusted for age, sex and educational level. 


\section{Chapter 4}

Cognition and violent behavior in psychotic disorders: a nationwide case-control study

Lamsma J

Cahn W

Fazel S

GROUP investigators

Schizophr Res Cogn 2020; 19: 100166. 


\section{Abstract}

\section{Background}

The excess risk of violence in psychotic disorders may partly be explained by impairments in executive functions (EFs) and theory of mind (ToM). However, previous studies have been limited by composite measures of EFs and small samples of inpatients.

\section{Methods}

Data were collected for the research project Genetic Risk and Outcome of Psychosis (GROUP). Patients with psychotic disorders $(N=891)$ were recruited from various care settings in the Netherlands. The following neuropsychological tests were administered (targeted cognitive function between parentheses): (i) Continuous Performance Test-HQ (inhibition); (ii) Response Shifting Task (cognitive flexibility); (iii) Wechsler Adult Intelligence Scale, Third Edition (WAIS-III) Block Design subtest (fluid intelligence); (iv) Neuropsychological Assessment Battery (NAB) Mazes Test (planning); (v) Degraded Facial Affect Recognition Task (affective ToM); and (vi) Hinting Task (cognitive ToM). Lifetime violence was ascertained from medical records and patient interviews. We used analysis of covariance to compare the mean scores of violent and nonviolent patients on each test, adjusting for age and gender.

\section{Results}

Violent patients performed significantly worse than nonviolent patients on the WAIS-III Block Design subtest $(F[1,847]=5.12, p=.024)$, NAB Mazes Test $(F[1,499]=5.32, p=.022)$ and Hinting Task $(F[1,839]=9.38, p=.002)$. For the other tests, the between-group differences were nonsignificant. Violent behavior explained no more than $1 \%$ of the variance in performance on each test.

\section{Conclusion}

Impairments in EFs and ToM are unlikely to provide useful targets for risk assessment and interventions. 


\section{Introduction}

People with psychotic disorders are at increased risk of violent behavior compared with the general population ${ }^{1,2}$. In a meta-analysis of 20 studies with a total of 18,423 cases and $1,714,904$ unaffected controls, the odds ratio for violence in schizophrenia was 5.5 (95\% confidence interval $[\mathrm{Cl}] 4.1-7.5)$ and in other psychotic disorders $4.9(95 \% \mathrm{Cl} 3.6-6.6)^{1}$. More recent studies have confirmed this risk using family-based designs ${ }^{3,4}$. Several risk factors for violence in psychotic disorders have been identified, including previous crime, symptoms of delusions and hallucinations, lack of insight and substance misuse ${ }^{5}$. Potentially important, but rarely studied, are impairments in executive functions (EFs) and theory of mind (ToM).

EFs are mental operations needed to direct behavior toward the realization of goals ${ }^{6}$. There are three elementary EFs: inhibition, working memory and cognitive flexibility. These combine to build the higher order EFs of fluid intelligence (i.e. reasoning and problem solving) and planning ${ }^{7}$. ToM is the ability to infer mental states (e.g. motivations, emotions) in oneself and others $^{8}$. Two types of ToM can be distinguished, according to whether the mental state being inferred is affective (affective ToM) or cognitive (cognitive ToM) in nature ${ }^{9}$.

Since EFs and ToM are essential for social adaptation, impairments in these cognitive functions are thought to underly violent behavior. However, causal mechanisms likely differ by $\mathrm{EF}^{10}$ and ToM type ${ }^{11}$. General population studies have repeatedly found that people who display violent behavior perform worse on neuropsychological tests of $\mathrm{EFs}^{12}$ and $\mathrm{ToM}^{13,14}$ than those who do not. A meta-analysis of 126 studies totaling 14,786 participants reported a significant inverse association between neuropsychological test performance and antisocial behavior (including violence) across EFs. This association had a medium effect size ( $d=0.4,95 \%$ $\mathrm{Cl} 0.4-0.5)^{10}$.

Impairments in EFs and ToM are a core feature of psychotic disorders ${ }^{15}$. An umbrella review of 10 meta-analyses found that individuals with schizophrenia performed between 0.5 and 1.5 standard deviations below unaffected controls on tests of $\mathrm{EFs}^{16}$. Meta-analyses of ToM 
have reported similar effect sizes ${ }^{17,18}$. By and large, EF and ToM deficits are present before illness onset ${ }^{15}$, stable over time ${ }^{19}$ and independent of psychotic symptoms ${ }^{20}$ and treatment with antipsychotic medication ${ }^{21}$. Furthermore, they are associated with adverse outcomes such as rehospitalization ${ }^{22}$, substance misuse ${ }^{23}$ and low educational attainment ${ }^{24}$.

Based on this, it may be hypothesized that EF and ToM impairments partly explain the excess risk of violent behavior in psychotic disorders. The few studies investigating this hypothesis have produced mixed results for $\mathrm{EFs}^{25-29}$ and affective $\mathrm{ToM}^{30-33}$. However, these studies were limited by small samples of male inpatients or prisoners, a single data source of violent behavior and composite measures of EFs. To our knowledge, there has only been one study of cognitive ToM. This study reported a significant association between higher scores on the Unexpected Transfer Task ${ }^{34}$ and lifetime violence (relative risk $=1.2, p<.05$ ), ascertained from medical records and interviews with hospital staff, in patients with schizophrenia. This finding may be attributed to selection bias: the sample size was small $(N=24)$, and the violent patients were recruited from a high-security psychiatric hospital ${ }^{30}$.

To address the limitations of previous studies, we have investigated the association between neuropsychological test performance and a sensitive marker of violent behavior for a comprehensive set of EFs and cognitive and affective ToM in a large nationwide sample of people with psychotic disorders.

\section{Methods}

\section{Setting and participants}

Data (release 5.0) were collected as part of a larger project, called Genetic Risk and Outcome in Psychosis (GROUP). GROUP is conducted by the psychiatry departments of 4 university medical centers (i.e. Amsterdam Medical Center, Maastricht University Medical Center+, University Medical Center Groningen, University Medical Center Utrecht) and their affiliated 
mental health centers $(k=36)$ in the Netherlands. Together, these centers provide access to inpatient or outpatient treatment to approximately $75 \%$ of the Dutch population. Assessments took place at baseline (2004) and after three and six years of follow-up. Eligible patients were identified by screening clinicians' caseloads for the following criteria: (i) age between 16 and 50; (ii) good command of the Dutch language; and (iii) Diagnostic and Statistical Manual of Mental Disorders, Fourth Edition, Text Revision (DSM-IV-TR) ${ }^{35}$ diagnosis of schizophrenia or other psychotic disorder. There were no exclusion criteria. The protocol was approved centrally by the ethics committee of the University Medical Center Utrecht, and all patients gave written informed consent before the first assessment.

\section{Measures}

The psychometric properties of the instruments and training of research personnel have been described elsewhere ${ }^{36}$.

Neuropsychological tests

We chose tests whose targeted cognitive functions (in parentheses) are hypothetically related to violent behavior: (i) Continuous Performance Test-HQ (CPT-HQ) ${ }^{37}$ (inhibition); (ii) Response Shifting Task (RST) ${ }^{38}$ (cognitive flexibility); (iii) Wechsler Adult Intelligence Scale, Third Edition (WAIS-III) ${ }^{39}$ Block Design subtest (fluid intelligence); (iv) Neuropsychological Assessment Battery (NAB) ${ }^{40}$ Mazes Test (planning); (v) Degraded Facial Affect Recognition Task (DFAR) ${ }^{41}$ (affective ToM); and (vi) Hinting Task ${ }^{42}$ (cognitive ToM). The NAB Mazes Test was only administered at the third wave. Information about the testing procedure can be found in the supplement. 
Chapter 4

Symptom severity

Symptom severity was measured with the Positive and Negative Syndrome Scale (PANSS) ${ }^{43}$.

Substance misuse

The Substance Abuse Module of the Composite International Diagnostic Interview ${ }^{44}$ was used to establish a lifetime history of substance misuse. We defined alcohol misuse as an average intake of more than 18 standard drinks per week for men and more than 12 standard drinks per week for women during a minimum period of 2 weeks in the past year or 4 weeks at any other point in the past. These cutoffs reflect the median of several national guidelines and a consistent 1.5:1 male to female consumption ratio ${ }^{45}$. For other substances, misuse referred to a DSM-IV-TR diagnosis of abuse or dependence.

\section{Violent behavior}

Violent behavior, defined as the use of physical force with the intention to harm another person, was assessed with the Life Chart Schedule (LCS) ${ }^{46}$. The reference period was the lifetime. The LCS was filled out based on review of medical records and interviews with the patient and, if possible, one or both parents.

\section{Statistical analysis}

In the analyses involving the NAB Mazes Test, we used age, PANSS total score and educational level at the third wave. Otherwise, baseline data were used. Higher scores on all tests reflected better performance, apart from certain subscales of the CPT-HQ (i.e. number of commission errors) and RST (i.e. accuracy cost, reaction time cost). Therefore, these scores were 
reversed for the current analyses. For the CPT-HQ and RST, we created composite scores by transforming the scores on the subscales to $z$-scores and then averaging the $z$-scores. This method for creating composite scores is widely used ${ }^{47,48}$. To reduce confounding by impairments in face recognition ability, patients with scores below 18 on the Benton Facial Recognition Test ${ }^{49}$ were excluded from the analyses with the DFAR.

Depending on the scale of measurement, we assessed differences between violent and nonviolent patients on descriptive characteristics with the $\chi^{2}$-test (nominal) or $t$-test (continuous). Analysis of covariance (ANCOVA) was used to compare the mean scores of violent and nonviolent patients on each neuropsychological test. For theoretical reasons, we included age ${ }^{50}$ and gender ${ }^{51}$ as covariates. We only analyzed patients with available data on all model variables. This reduced the total number of patients from 1,013 to 891 .

To evaluate the robustness of the results, we repeated the analyses after separately removing: (i) patients whose violence did not result in injury; (ii) patients who had been violent before illness onset; (iii) patients with PANSS total scores of 95 or higher, indicative of "marked or severe illness" 52 ; (iv) patients who had misused substances; and (v) patients who had not completed secondary education.

All models satisfied the assumptions of ANCOVA (e.g. homoscedasticity, homogeneity of regression slopes). The level of statistical significance was set at $5 \%$. Analyses were carried out in SPSS 21.0.

\section{Results}

Table 1 shows the demographic and clinical characteristics of the patients $(N=891)$ at baseline. The mean age was $27.2(S D=7.0)$. Most patients were male $(n=688,77 \%)$ and had received a diagnosis of schizophrenia ( $n=615,69 \%)$. The prevalence of violent behavior was $21 \%$ ( $n=$ 183). Violent patients were significantly younger ( $t[889]=2.90, p=.004)$, had higher PANSS 
Chapter 4

total scores $(t[809]=3.80, p<.001)$ and were more likely to have misused substances $\left(\chi^{2}[1]\right.$ $=4.26, p=.039)$ than nonviolent patients.

Violent patients performed worse than nonviolent patients on most neuropsychological tests (Table 2). On average, they had fewer hits and longer reaction times for hits on the CPT-HQ, higher accuracy and reaction time cost scores on the RST and lower scores on the WAIS-III Block Design subtest, NAB Mazes Test and Hinting Task. The mean number of commission errors on the CPT-HQ was lower and percentage of correctly identified emotions on the DFAR was higher in violent patients, indicating better performance. The between-group differences reached statistical significance for the WAIS-III Block Design subtest $(F[1,847]=5.1, p=.024)$, NAB Mazes Test $(F[1,499]=5.32, p=.022)$ and Hinting Task $(F[1,839]=9.4, p=.002)$. Effect sizes were small: violent behavior explained $1 \%$ or less of the variance in performance on each test.

We observed no material differences in results when restricting the analyses to patients whose violence resulted in injury (Table S2), patients who had only been violent after illness onset (Table S3), patients with PANSS total scores below 95 (Table S4), patients without substance misuse (Table S5) or patients who had completed secondary education (Table S6).

\section{Discussion}

In a nationwide sample of 891 patients with psychotic disorders, we have investigated the association between neuropsychological test performance and lifetime violence for a comprehensive range of EFs and cognitive and affective theory of mind. Violent patients performed significantly worse than nonviolent patients on tests of fluid intelligence (i.e. WAIS-III Block Design subtest), planning (i.e. NAB Mazes Test) and cognitive ToM (i.e. Hinting Task). However, effect sizes were small. 
Table 1. Baseline characteristics of patients with psychotic disorders.

\begin{tabular}{|c|c|c|c|c|}
\hline & \multicolumn{2}{|c|}{ Lifetime violence } & \multirow[b]{2}{*}{ Test statistic $(d f)$} & \multirow[b]{2}{*}{$p$} \\
\hline & Yes $(n=183)$ & No $(n=708)$ & & \\
\hline \multicolumn{5}{|l|}{ Demographic characteristics } \\
\hline Age, mean (SD) in years & $25.9(6.3)$ & $27.6(7.2)$ & $t(889)=2.90$ & .004 \\
\hline Male, $n(\%)$ & $145(79)$ & $543(77)$ & $\chi^{2}(1)=0.53$ & ns \\
\hline Caucasian, $n(\%)$ & $137(76)$ & $559(80)$ & $\chi^{2}(8)=9.66$ & ns \\
\hline Completed secondary education, $n(\%)$ & $146(80)$ & $620(88)$ & $\chi^{2}(1)=8.17$ & .004 \\
\hline \multicolumn{5}{|l|}{ Clinical characteristics } \\
\hline DSM-IV-TR diagnosis & & & $\chi^{2}(3)=6.80$ & ns \\
\hline Schizophrenia, $n$ (\%) & $136(74)$ & $479(68)$ & & \\
\hline Schizoaffective disorder, $n$ (\%) & $18(10)$ & $90(13)$ & & \\
\hline Psychotic Disorder NOS, $n$ (\%) & $18(10)$ & $57(8)$ & & \\
\hline Other, $n(\%)$ & $11(6)$ & $82(12)$ & & \\
\hline Age of onset, mean (SD) in years & $21.7(5.6)$ & $23.4(6.6)$ & $t(889)=3.37$ & .001 \\
\hline PANSS total score, mean (SD) & $58.9(17.1)$ & $53.5(16.2)$ & $t(809)=3.80$ & $<.001$ \\
\hline Substance misuse, $n(\%)$ & $118(68)$ & $405(60)$ & $\chi^{2}(1)=4.26$ & .039 \\
\hline
\end{tabular}

$d f$, degrees of freedom; SD, standard deviation; ns, nonsignificant; DSM-IV-TR, Diagnostic and Statistical Manual of Mental Disorders, Fourth Edition, Text Revision; NOS, not otherwise specified; PANSS, Positive and Negative Syndrome Scale.

Due to missing data, the total number of patients varies per characteristic. 


\section{Chapter 4}

Table 2. Neuropsychological test performance in violent and nonviolent patients with psychotic disorders.

\begin{tabular}{|c|c|c|c|c|c|c|c|c|}
\hline \multirow[b]{2}{*}{ Targeted cognitive function } & \multirow[b]{2}{*}{ Neuropsychological test } & \multicolumn{2}{|c|}{ Unadjusted M (SD) } & \multicolumn{2}{|c|}{ Adjusted M (SE) ${ }^{\mathrm{a}}$} & \multirow[b]{2}{*}{$F(d f 1, d f 2)$} & \multirow[b]{2}{*}{$p$} & \multirow[b]{2}{*}{$\eta_{p}^{2}$} \\
\hline & & $\mathrm{V}(n=183)$ & $\mathrm{NV}(n=708)$ & $\mathrm{V}(n=183)$ & $\mathrm{NV}(n=708)$ & & & \\
\hline \multicolumn{9}{|l|}{ Executive functions } \\
\hline \multirow[t]{4}{*}{ Inhibition } & $\mathrm{CPT}-\mathrm{HQ}^{\mathrm{b}}$ & $0.01(0.52)$ & $0.00(0.55)$ & $0.02(0.04)$ & $-0.01(0.02)$ & $0.33(1,782)$ & ns & $<.001$ \\
\hline & Number of hits & $26.1(3.0)$ & $26.4(2.8)$ & & & & & \\
\hline & Mean reaction time hits ${ }^{c}$ & $44.0(8.3)$ & $42.8(8.7)$ & & & & & \\
\hline & Number of commission errors & $2.6(10.8)$ & $3.0(15.3)$ & & & & & \\
\hline \multirow[t]{3}{*}{ Cognitive flexibility } & $\mathrm{RST}^{\mathrm{b}}$ & $-0.05(0.79)$ & $0.01(0.75)$ & $-0.06(0.06)$ & $0.02(0.03)$ & $1.27(1,758)$ & ns & .002 \\
\hline & Accuracy cost & $24.4(25.5)$ & $22.2(23.6)$ & & & & & \\
\hline & Reaction time cost ${ }^{c}$ & $26.2(18.9)$ & $25.3(18.9)$ & & & & & \\
\hline Fluid intelligence & WAIS-III Block Design test & $38.2(17.5)$ & $41.0(16.9)$ & $37.8(1.3)$ & $41.1(0.7)$ & $5.12(1,847)$ & .024 & .006 \\
\hline Planning & NAB Mazes Test ${ }^{d}$ & $15.9(6.5)$ & $17.0(6.3)$ & $15.6(0.6)$ & $17.1(0.3)$ & $5.32(1,499)$ & .022 & .011 \\
\hline \multicolumn{9}{|l|}{ Theory of mind } \\
\hline Affective & DFAR & $69.1(10.2)$ & $68.9(10.7)$ & $69.0(0.8)$ & $68.9(0.4)$ & $0.01(1,761)$ & ns & $<.001$ \\
\hline Cognitive & Hinting Task & $17.0(2.9)$ & $17.7(2.7)$ & $17.0(0.2)$ & $17.7(0.1)$ & $9.38(1,839)$ & .002 & .011 \\
\hline
\end{tabular}

M, mean; SD, standard deviation; SE, standard error; V, violent; NV, nonviolent; df, degrees of freedom; CPT-HQ, Continuous Performance Test-HQ; ns, nonsignificant; RST, Response Shifting Task; WAIS-III, Wechsler Adult Intelligence Scale, Third Edition; NAB, Neuropsychological Assessment Battery; DFAR, Degraded Facial Affect Recognition Task.

Due to missing data, the total number of patients varies per test.

${ }^{a}$ Adjusted for age and sex.

${ }^{\mathrm{b}}$ Average of subscale $z$-scores.

c In centiseconds.

${ }^{d}$ Administered six years after baseline. 
Violent behavior in people with impaired fluid intelligence may be a maladaptive solution to ${ }^{53}$ or consequence of increased stress responsivity in provocative situations ${ }^{54}$. Planning deficiencies increase the probability of violence by negatively affecting a person's ability to assess the possible consequences of his or her actions ${ }^{55}$. Impairments in ToM may lead to violence through misinterpretation of social cues ${ }^{11}$, underregulation of negative emotions ${ }^{56}$, blurring of self-other boundaries ${ }^{11}$ or lack of empathy ${ }^{57}$. There was no significant difference between violent and nonviolent patients in affective ToM, which is arguably more important for the last three than cognitive ToM. This lends weight to misinterpretation of social cues - insofar they relate to other people's cognitive mental states - as a reason for violent behavior in psychotic disorders. Cognitive ToM also subserves insight ${ }^{58}$ and, relatedly, treatment adherence ${ }^{59}$. Insight refers to a person's acknowledgement of having an illness that requires treatment ${ }^{60}$. A person lacking insight may not seek or adhere to treatment, thereby allowing psychotic symptoms to persist or worsen ${ }^{61}$. Furthermore, a strong conviction that delusions or hallucinations are real may bring a person to act on them ${ }^{62}$. Alternative explanations for the findings are confounding by biological (e.g. genetics, neurobiological abnormalities) or early environmental (e.g. poor nutrition, maltreatment) risk factors ${ }^{63}$.

There are several limitations to this study. First, causality cannot be inferred because violent behavior preceded test administration by a potentially long period of time. However, the relative stability of cognition makes this less problematic. Second, the LCS does not distinguish between community and inpatient violence, which may have different cognitive correlates ${ }^{53}$. It has also been suggested that cognitive impairment is more pronounced in patients who persistently display violent behavior from an early age than in those who become violent after illness onset ${ }^{64}$. However, we found similar results in the latter. Third, most patients had used antipsychotic medication. This may have attenuated associations, as some antipsychotics most notably clozapine - have been shown to reduce violence ${ }^{65,66}$. However, reported improvements in $\mathrm{EFs}^{67}$ and $\mathrm{ToM}^{68}$ after treatment with antipsychotics are usually too small to be considered clinically significant. For that reason, we expect attenuation to have been negligible. Fourth, neuropsychological tests have limited construct and ecological validity. The construct validity, or the degree to which an instrument measures what it is designed to measure, of many tests is lowered by systematic variance in performance that is attributable to 
nontargeted cognitive functions. For example, the WAIS-III Block Design subtest not only measures fluid intelligence but also visual-spatial skills ${ }^{69}$. Ecological validity concerns the extent to which the score obtained with an instrument can be generalized to real-world settings $^{70}$. An individual who performs at or above the normative level on a test may still experience difficulties in everyday life when requiring the cognitive function targeted by that test. For one reason, the demands placed on cognitive functions in real-world settings are more complex than in experimental settings. For another, neuropsychological tests are designed to detect clinically significant impairments in cognitive functions. This is relevant, as cognitive impairments in violent individuals are often subclinical ${ }^{12}$. Fifth, cognitive ToM was assessed verbally. Given that poverty of speech is a prominent symptom of psychotic disorders, this may have introduced bias ${ }^{71}$. However, a meta-analysis of 29 studies found that individuals with schizophrenia $(N=1,518)$ performed similarly on verbal $(d=1.2,95 \% \mathrm{Cl} 1.0-1.5)$ and nonverbal $(d=1.3,95 \% \mathrm{Cl} 1.0-1.5)$ tests of cognitive ToM $^{18}$. Finally, the CPT-HQ measures only one of three types of inhibition, namely selective attention. The other types are cognitive inhibition and self-control. Selective attention allows one to focus on a particular stimulus, while ignoring others. Cognitive inhibition involves the suppression of irrelevant thoughts, typically to support working memory. Self-control refers to goal-oriented regulation of thoughts and emotions ${ }^{7}$. As such, self-control may be expected to be most directly related to violent behavior. However, selective attention and self-control are highly correlated ${ }^{72}$ and recruit largely the same neural systems ${ }^{73}$.

The findings of this study provide little justification for using EFs and ToM as targets for risk assessment and interventions. While most risk assessment tools do not contain items for cognitive functions ${ }^{74}$, the small effect sizes suggest that additional items for fluid intelligence, planning and cognitive ToM would confer marginal improvements at most. For the same reason, interventions aimed at improving fluid intelligence, planning and cognitive ToM are unlikely to prevent violent behavior on their own. To improve understanding of causal mechanisms, future studies should use prospective designs and test for additional confounders and mediators. 
In conclusion, we have found significant but small associations between poorer neuropsychological test performance and violent behavior in psychotic disorders for three cognitive functions: fluid intelligence, planning and cognitive ToM. This provides some empirical support for cognitive models of violent behavior. At the same time, the findings suggest that impairments in EFs and ToM have little to no value for risk assessment and interventions. 


\section{References}

1. Fazel S, Gulati G, Linsell L, et al. Schizophrenia and violence: systematic review and metaanalysis. PLoS Med 2009; 6: e1000120.

2. Large MM, Nielssen O. Violence in first-episode psychosis: a systematic review and metaanalysis. Schizophr Res 2011; 125: 209-220.

3. Fazel S, Wolf A, Palm C, et al. Violent crime, suicide, and premature mortality in patients with schizophrenia and related disorders: a 38-year total population study in Sweden. Lancet Psychiatry 2014; 1: 44-54.

4. Sariaslan A, Larsson H, Fazel S. Genetic and environmental determinants of violence risk in psychotic disorders: a multivariate quantitative genetic study of 1.8 million Swedish twins and siblings. Mol Psychiatry 2016; 21: 1251-1256.

5. Witt K, Van Dorn RA, Fazel S. Risk factors for violence in psychosis: systematic review and meta-regression analysis of 110 studies. PLoS One 2013; 8: e55942.

6. Miller EK, Wallis JD. Executive function and higher-order cognition: definition and neural substrates. In LR Squire (Ed), Encyclopedia of neuroscience (99-104). Academic Press, 2009.

7. Diamond A. Executive Functions. Annu Rev Psychol 2003; 64: 135-168.

8. Fonagy $\mathrm{P}$, Allison $\mathrm{E}$. What is mentalization? The concept and its foundations in developmental research. In N Midgley, I Vrouva (Eds), Minding the child: mentalizationbased interventions with children, young people and their families (11-34). Routledge, 2012.

9. Shamay-Tsoory SG, Harari H, Aharon-Peretz J, et al. The role of the orbitofrontal cortex in affective theory of mind deficits in criminal offenders with psychopathic tendencies. Cortex 2010; 46: 668-677.

10. Raine A. From genes to brain to antisocial behavior. Curr Dir Psychol Sci 2008; 17: 323328.

11. Adshead G, Moore E, Humphrey M, et al. The role of mentalising in the management of violence. Adv Psychiatr Treat 2013; 19: 67-76. 
12. Ogilvie JM, Stewart AL, Chan RC, et al. Neuropsychological measures of executive function and antisocial behavior: a meta-analysis. Criminology 2011; 49: 1063-1107.

13. Hoaken PN, Allaby DB, Earle J. Executive cognitive functioning and the recognition of facial expressions of emotion in incarcerated violent offenders, non-violent offenders, and controls. Aggress Behav 2007; 33: 412-421.

14. Nyline B, Softas-Nall L, Peterson E. Inaccuracies in facial recognition of fear and sadness for male domestic violence offenders. Open J Soc Sci 2018; 6: 37-51.

15. Kahn RS, Keefe RS. Schizophrenia is a cognitive illness: time for a change in focus. JAMA Psychiatry 2013; 70: 1107-1112.

16. Reichenberg A, Harvey PD. Neuropsychological impairments in schizophrenia: integration of performance-based and brain imaging findings. Psychol Bull 2007; 133: 833-858.

17. Chung YS, Barch D, Strube M. A meta-analysis of mentalizing impairments in adults with schizophrenia and autism spectrum disorder. Schizophr Bull 2014; 40: 602-616.

18. Sprong M, Schothorst $\mathrm{P}$, Vos $\mathrm{E}$, et al. Theory of mind in schizophrenia: meta-analysis. Br J Psychiatry 2007; 191: 5-13.

19. Reichenberg A. The assessment of neuropsychological functioning in schizophrenia. Dialogues Clin Neurosci 2011; 12: 383-392.

20. Nieuwenstein MR, Aleman A, de Haan EH. Relationship between symptom dimensions and neurocognitive functioning in schizophrenia: a meta-analysis of WCST and CPT studies. J Psychiatr Res 2001; 35: 119-125.

21. Mishara AL, Goldberg TE. A meta-analysis and critical review of the effects of conventional neuroleptic treatment on cognition in schizophrenia: opening a closed book. Biol Psychiatry 2004; 55: 1013-1022.

22. Harvey PD, Loewenstein DA, Czaja SJ. Hospitalization and psychosis: influences on the course of cognition and everyday functioning in people with schizophrenia. Neurobiol Dis 2013; 53: 18-25.

23. Eack SM, Hogarty SS, Greenwald DP, et al. Cognitive Enhancement Therapy in substance misusing schizophrenia: results of an 18-month feasibility trial. Schizophr Res 2015; 61: 478-83.

24. Keefe RS. Should cognitive impairment be included in the diagnostic criteria for schizophrenia? World Psychiatry 2008; 7: 22-28. 
25. Barkataki I, Kumari V, Das M, et al. A neuropsychological investigation into violence and mental illness. Schizophr Res 2005; 74: 1-13.

26. Fullam RS, Dolan MC. Executive function and in-patient violence in forensic patients with schizophrenia. Br J Psychiatry 2008; 193: 247-253.

27. Lapierre D, Braun CM, Hodgins S, et al. Neuropsychological correlates of violence in schizophrenia. Schizophr Bull 1995; 21: 253-262.

28. Krakowski MI, De Sanctis P, Foxe JJ, et al. Disturbances in response inhibition and emotional processing as potential pathways to violence in schizophrenia: a high-density event-related potential study. Schizophr Bull 2016; 42: 963-974.

29. Serper M, Beech DR, Harvey PD, et al. Neuropsychological and symptom predictors of aggression on the psychiatric inpatient service. J Clin Exp Neuropsychol 2008; 30: 700709.

30. Abu-Akel A, Abushua'leh K. 'Theory of mind' in violent and nonviolent patients with paranoid schizophrenia. Schizophr Res 2004; 69: 45-53.

31. Frommann N, Stroth S, Brinkmeyer J, et al. Facial affect recognition performance and event-related potentials in violent and non-violent schizophrenia patients. Neuropsychobiology 2013; 68: 139-145.

32. Silver H, Goodman C, Knoll G, et al. Schizophrenia patients with a history of severe violence differ from nonviolent schizophrenia patients in perception of emotions but not cognitive function. J Clin Psychiatry 2005; 66: 300-308.

33. Weiss EM, Kohler CG, Nolan KA, et al. The relationship between history of violent and criminal behavior and recognition of facial expression of emotions in men with schizophrenia and schizoaffective disorder. Aggress Behav 2006; 32: 187-194.

34. Perner J, Wimmer H. "John thinks that Mary thinks that. . ." attribution of second-order false beliefs by 5- to 10-year-old children. J Exp Child Psychol 1985; 39: 437-471.

35. American Psychiatric Association. Diagnostic and Statistical Manual of Mental Disorders (4th edition, text revision). Author, 2000.

36. Korver N, Quee PJ, Boos HB, et al. Genetic Risk and Outcome of Psychosis (GROUP), a multi-site longitudinal cohort study focused on gene-environment interaction: objectives, sample characteristics, recruitment and assessment methods. Int J Methods Psychiatric Res 2012; 21: 205-221. 
37. Nuechterlein KH, Dawson ME. Information processing and attentional functioning in the developmental course of schizophrenic disorders. Schizophr Bull 1984; 10: 160-203.

38. Bilder RM, Turkel E, Lipschutz-Broch L, et al. Antipsychotic medication effects on neuropsychological functions. Psychopharmacol Bull 1992; 28: 353-366.

39. Wechsler D. Wechsler Adult Intelligence Scale (3rd edition). Psychological Corporation, 1997.

40. Stern RA, White T. Neuropsychological Assessment Battery. Psychological Assessment Resources, 2003.

41. van 't Wout $M$, Aleman A, Kessels RP, et al. Emotional processing in a non-clinical psychosis-prone sample. Schizophr Res 2004; 68: 271-281.

42. Corcoran R, Mercer G, Frith CD. Schizophrenia, symptomatology and social inference: investigating "theory of mind" in people with schizophrenia. Schizophr Res 1995; 17: 513.

43. Kay SR, Fiszbein A, Opler LA. The positive and negative syndrome scale (PANSS) for schizophrenia. Schizophr Bull 1987; 13: 261-276.

44. World Health Organization. Composite International Diagnostic Interview. WHO, 1990.

45. Furtwaengler NA, de Visser RO. Lack of international consensus in low-risk drinking guidelines. Drug Alcohol Rev 2013; 32: 11-18.

46. Susser E, Finnerty M, Mojtabai R, et al. Reliability of the life chart schedule for assessment of the long-term course of schizophrenia. Schizophr Res 2000; 42: 67-77.

47. Harrison JE, Lophaven S, Olsen CK. Which cognitive domains are improved by treatment with vortioxetine? Int J Neuropsychopharmacol 2016; 19: pyw054.

48. Mancuso F, Horan W, Kern RS, et al. Social cognition in psychosis: multidimensional structure, clinical correlates, and relationships with functional outcome. Schizophr Res 2011; 125: 143-151.

49. Benton AL, Sivan AB, Hamsher KS, et al. Benton's Test of Facial Recognition. Oxford University Press: New York, 1983.

50. Henry JD, Phillips LH, Ruffman T, et al. A meta-analytic review of age differences in theory of mind. Psychol Aging 2013; 28: 826-839. 
51. Longenecker J, Dickinson D, Weinberger DR, et al. Cognitive differences between men and women: a comparison of patients with schizophrenia and healthy volunteers. Schizophr Res 2010; 120: 234-235.

52. Leucht S, Kane JM, Kissling W, et al. What does the PANSS mean? Schizophr Res 2005; 79: 231-238.

53. Weiss EM. Neuroimaging and neurocognitive correlates of aggression and violence in schizophrenia. Scientifica 2012: 158646.

54. Sandi C, Haller J. Stress and the social brain: behavioural effects and neurobiological mechanisms. Nat Rev Neurosci 2015; 16: 290-304.

55. Meijers J, Harte JM, Meynen G, et al. Differences in executive functioning between violent and non-violent offenders. Psychol Med 2017; 47: 1784-1793.

56. Fonagy $\mathrm{P}$, Luyten $\mathrm{P}$. A developmental, mentalization-based approach to the understanding and treatment of borderline personality disorder. Dev Psychopathol 2019; 21: $1355-1381$.

57. Hooker $\mathrm{Cl}$, Verosky SC, Germine LT, Knight RT, et al. Mentalizing about emotion and its relationship to empathy. Soc Cogn Affect Neurosci 2018; 3: 204-217.

58. Ng R, Fish S, Granholm E. Insight and theory of mind in schizophrenia. Psychiatry Res 2015; 225: 169-174.

59. Shad MU, Tamminga CA, Cullum $M$, et al. Insight and frontal cortical function in schizophrenia: a review. Schizophr Res 2006; 86: 54-70.

60. Greenberger C, Serper MR. Examination of clinical and cognitive insight in acute schizophrenia patients. J Nerv Ment Dis 2010; 198: 465-469.

61. Higashi K, Medic G, Littlewood KJ, et al. Medication adherence in schizophrenia: factors influencing adherence and consequences of nonadherence, a systematic literature review. Ther Adv Psychopharmacol 2013; 3: 200-218.

62. Bjørkly S. Empirical evidence of a relationship between insight and risk of violence in the mentally ill - a review of the literature. Aggress Violent Behav 2006; 11: 414-423.

63. Lamsma J, Harte JM. Violence in psychosis: conceptualizing its causal relationship with risk factors. Aggress Violent Behav 2015; 24: 75-82.

64. Hodgins S, Klein S. New clinically relevant findings about violence by people with schizophrenia. Can J Psychiatr 2017; 62: 86-93. 
65. Bhavsar V, Kosidou K, Widman L, et al. Clozapine treatment and offending: a withinsubject study of patients with psychotic disorders in Sweden. Schizophr Bull. Advance online publication: https://doi.org/10.1093/schbul/sbz055.

66. Fazel S, Zetterqvist J, Larsson $\mathrm{H}$, et al. Antipsychotics, mood stabilisers, and risk of violent crime. Lancet 2014; 384: 1206-1214.

67. Mishara AL, Goldberg TE. A meta-analysis and critical review of the effects of conventional neuroleptic treatment on cognition in schizophrenia: opening a closed book. Biol Psychiatry 2004; 55: 1013-1022.

68. Kucharska-Pietura K, Mortimer A. Can antipsychotics improve social cognition in patients with schizophrenia? CNS Drugs 2013; 27: 335-343.

69. Lera-Miguel S, Andrés-Perpiñá S, Calvo R, et al. Early-onset bipolar disorder: how about visual-spatial skills and executive functions? Eur Arch Psychiatry Clin Neurosci 2011; 261: 195-203.

70. Dawson DR, Marcotte TD. Special issue on ecological validity and cognitive assessment. Neuropsychol Rehabil 2017; 27: 599-602.

71. Sarfati $Y$, Hardy-Baylé M-C, Brunet E, et al. Investigating theory of mind in schizophrenia: influence of verbalization in disorganized and non-disorganized patients. Schizophr Res 1999; 37: 183-190.

72. Friedman NP, Miyake A. The relations among inhibition and interference control functions: a latent-variable analysis. J Exp Psychol 2004; 133: 101-135.

73. Cohen AS, Forbes CB, Mann MC, et al. Specific cognitive deficits and differential domains of social functioning impairment in schizophrenia. Schizophr Res 2006; 81: 227-238.

74. Singh JP, Serper M, Reinharth J, et al. Structured assessment of violence risk in schizophrenia and other psychiatric disorders: a systematic review of the validity, reliability, and item content of 10 available instruments. Schizophr Bull 2011; 37: 899912. 
Chapter 4

Supplementary material 


\section{Neuropsychological assessment}

The testing procedure at baseline is summarized in Table S1. Patients were assessed on all domains of the Measurement and Treatment Research to Improve Cognition in Schizophrenia consensus battery, except "visual learning and memory" ${ }^{1}$. Testing was done in a single session and took about two hours in total. Breaks between tests were offered whenever the patient showed signs of fatigue. Below follows a description of the neuropsychological tests used in the present study.

Inhibition was measured with the Continuous Performance Test- $\mathrm{HQ}^{2}$. A total of 300 letters appear in quasi-random order on a computer screen and the subject is instructed to press the space bar on a keyboard each time the letter " $Q$ " is preceded by the letter " $H$ " $(k=28)$. Letters are presented for $150 \mathrm{~ms}$, with intervals of $850 \mathrm{~ms}$. We used three performance indicators: (i) the number of hits (i.e. correct positive responses); (ii) the mean reaction time for hits; and (iii) the number of commission errors (i.e. false positive responses).

The Response Shifting Task (RST), a modified version of the Competing Programs Task ${ }^{3}$, was used to assess cognitive flexibility. The RST requires set-shifting between imitation and reversal response rules. The stimulus words "left" and "right" appear quasi-randomly on a computer screen for $3 \mathrm{~s}$ and should be followed each time by a press on either the left (i.e. " $z$ ") or right (i.e. "/") side of the keyboard. In the imitation condition, the subject must press the key congruent with the meaning of the stimulus (e.g. " $\mathrm{z}$ " when the word "left" appears). In the reversal condition, the subject must press the key incongruent with the meaning of the stimulus (e.g. "I" when the word "left" appears). Each condition is presented twice in alternating blocks, which end after 20 responses or 8 consecutive correct responses. The subject has to rely on feedback, shown for $1 \mathrm{~s}$ after each response, to deduce the response rule of each block. Two performance indicators were used: (i) accuracy cost (i.e. the absolute difference between the percentage of correct responses in the imitation condition and the percentage of correct responses in the reversal condition); and (ii) reaction time cost (i.e. the absolute difference between the mean reaction time in the imitation condition and the mean reaction 
time in the reversal condition). The first response in each block, responses preceded by errors, responses with a reaction time shorter than $150 \mathrm{~ms}$ and reaction times for incorrect responses were excluded.

Fluid intelligence was measured with the Block Design subtest of the Wechsler Adult Intelligence Scale, Third Edition ${ }^{4}$. The subject is asked to arrange 4 to 16 red and white square blocks in color patterns that match those shown on cards or made by the examiner. Each trial has a specified time limit (range: 90-240 s). Patterns that are laid incorrectly or outside the time limit are scored with 0 points. Scores for correct patterns depend on the number of tries and time to completion. If the subject lays 5 incorrect patterns in succession, the test is terminated and he or she receives a total score of 0 points. The maximum score is 68 points.

The Mazes Test of the Neuropsychological Assessment Battery ${ }^{5}$ was used to assess planning ability. The subject has to complete 7 progressively difficult mazes with time limits (range: 30 $240 \mathrm{~s}$ ) imposed on each. An uncompleted maze is scored with 0 points. Time to completion is divided in intervals that are scored with 1 to 3 or 5 points. If the subject fails to complete 3 mazes in a row, the test is terminated and he or she receives a total score of 0 points. The maximum score is 26 points.

The Degraded Facial Affect Recognition Task ${ }^{6,7}$ was used to measure affective theory of mind (ToM). Photographs of four actors (two male and two female) with angry, happy, fearful and neutral faces appear in random order on a computer screen. The subject is instructed to identify the emotion expressed in each face by pressing the key with the corresponding number (i.e. "1"-“4"). There are 16 representations of each emotion, making the total number of trials 64. To increase the contribution of top-down processes, such as attention, mental imagery and feature binding, the photographs are passed through a filter that reduces visual contrast by $30 \%$. For the same reason, half of the trials for angry, happy and fearful faces are displayed at $75 \%$ intensity ${ }^{7}$. The percentage of correctly identified emotions was used as the performance indicator. 
Face recognition ability was assessed with the short form of the Benton Facial Recognition Test (BFRT) ${ }^{8}$. The BFRT uses photographs of male and female faces with hair and clothing cropped out. The subject is asked to match a target face with 1 or 3 out of 6 test faces in 13 trials. Correctly matched faces are scored with 1 point. The maximum score is 27 points.

Cognitive ToM was measured with the Hinting Task ${ }^{9}$. The Hinting Task consists of 10 short stories, each involving an interaction between two characters, that are read aloud. Each story ends with one of the characters making an implicit statement. The subject is then asked to explain what the character really meant. An immediate correct answer is scored with 2 points. In case of an incorrect response, the subject is given a hint. Subsequent responses are scored with 1 point, if correct, or 0 points, if incorrect. 
Table S1. Neuropsychological tests administered at baseline.

\begin{tabular}{|c|c|c|}
\hline Neuropsychological test & Targeted cognitive function & Reference \\
\hline Word Learning Task (immediate recall)a & Short-term memory & Brand and Jolles (1985) \\
\hline Response Shifting Task ${ }^{a, b}$ & Cognitive flexibility & Bilder et al. (1992) \\
\hline Continuous Performance Test- $\mathrm{HQ}^{\mathrm{a}, \mathrm{b}}$ & Inhibition & Neuechterlein and Dawson (1984) \\
\hline WAIS-III (Digit-Symbol Coding) & Processing speed & Wechsler (1997) \\
\hline Word Learning Task (delayed recall and recognition) ${ }^{a}$ & Short-term memory & Brand and Jolles (1985) \\
\hline Degraded Facial Affect Recognition Task ${ }^{a, b}$ & Affective ToM & van 't Wout et al. (2004) \\
\hline Benton Facial Recognition Test ${ }^{b}$ & Face recognition & Benton et al. (1983) \\
\hline WAIS-III (Information) & Semantic memory & Wechsler (1997) \\
\hline WAIS-III (Arithmetic) & Working memory & Wechsler (1997) \\
\hline WAIS-III (Block Design) & Fluid intelligence & Wechsler (1997) \\
\hline Hinting Task & Cognitive ToM & Corcoran et al. (1995) \\
\hline
\end{tabular}

WAIS-III, Wechsler Adult Intelligence Scale, Third Edition; ToM, theory of mind.

Neuropsychological tests are listed in the order in which they were administered. Parentheses indicate subtests.

${ }^{a}$ Administered using E-Prime 1.3 (Psychology Software Tools Inc., Pittsburgh).

${ }^{\mathrm{b}}$ Included in the present study. 
Table S2. Neuropsychological test performance in violent and nonviolent patients with psychotic disorders, including only patients with violence that resulted in injury.

\begin{tabular}{|c|c|c|c|c|c|c|c|c|}
\hline \multirow[b]{2}{*}{ Targeted cognitive function } & \multirow[b]{2}{*}{ Neuropsychological test } & \multicolumn{2}{|c|}{ Unadjusted M (SD) } & \multicolumn{2}{|c|}{ Adjusted M (SE) } & \multirow[b]{2}{*}{$F(d f 1, d f 2)$} & \multirow[b]{2}{*}{$p$} & \multirow[b]{2}{*}{$\eta_{p}^{2}$} \\
\hline & & $\mathrm{V}(n=56)$ & $\mathrm{NV}(n=708)$ & $\mathrm{V}(n=56)$ & $\mathrm{NV}(n=708)$ & & & \\
\hline \multicolumn{9}{|l|}{ Executive functions } \\
\hline \multirow[t]{4}{*}{ Inhibition } & $\mathrm{CPT}-\mathrm{HQ}^{\mathrm{b}}$ & $0.02(0.48)$ & $0.00(0.55)$ & $0.05(0.08)$ & $0.00(0.02)$ & $0.40(1,670)$ & ns & .001 \\
\hline & Number of hits & $26.0(3.7)$ & $26.4(2.8)$ & & & & & \\
\hline & Mean reaction time hits ${ }^{c}$ & $44.1(8.9)$ & $42.8(8.7)$ & & & & & \\
\hline & Number of commission errors & $2.1(5.1)$ & $3.0(15.3)$ & & & & & \\
\hline \multirow[t]{3}{*}{ Cognitive flexibility } & $\mathrm{RST}^{\mathrm{b}}$ & $-0.17(0.76)$ & $0.01(0.75)$ & $-0.20(0.11)$ & $0.01(0.03)$ & $3.69(1,654)$ & ns & .006 \\
\hline & Accuracy cost & $28.7(29.5)$ & $22.2(23.6)$ & & & & & \\
\hline & Reaction time $\operatorname{cost}^{\mathrm{c}}$ & $27.4(17.2)$ & $25.3(18.9)$ & & & & & \\
\hline Fluid intelligence & WAIS-III Block Design subtest & $33.9(17.5)$ & $41.0(16.9)$ & $33.2(2.4)$ & $41.1(0.6)$ & $10.35(1,724)$ & .001 & .014 \\
\hline Planning & NAB Mazes Test ${ }^{d}$ & $15.0(7.0)$ & $17.0(6.3)$ & $14.4(1.1)$ & $17.0(0.3)$ & $5.21(1,424)$ & .023 & .012 \\
\hline \multicolumn{9}{|l|}{ Theory of mind } \\
\hline Affective & DFAR & $68.4(10.3)$ & $68.9(10.7)$ & $68.3(1.5)$ & $68.9(0.4)$ & $0.13(1,654)$ & ns & $<.001$ \\
\hline Cognitive & Hinting Task & $16.9(3.5)$ & $17.7(2.7)$ & $17.0(0.4)$ & $17.7(0.1)$ & $3.40(1,719)$ & ns & .005 \\
\hline
\end{tabular}

M, mean; SD, standard deviation; SE, standard error; V, violent; NV, nonviolent; df, degrees of freedom; CPT-HQ, Continuous Performance Test-HQ; ns, nonsignificant; RST, Response Shifting Task; WAIS-III, Wechsler Adult Intelligence Scale, Third Edition; NAB, Neuropsychological Assessment Battery; DFAR, Degraded Facial Affect Recognition Task.

Due to missing data, the total number of patients varies per test.

adjusted for age and sex.

Average of subscale $z$-scores.

c In centiseconds.

${ }^{\mathrm{d}}$ Administered six years after baseline. 


\section{Chapter 4}

Table S3. Neuropsychological test performance in violent and nonviolent patients with psychotic disorders, excluding patients with violence before illness onset.

\begin{tabular}{|c|c|c|c|c|c|c|c|c|}
\hline \multirow[b]{2}{*}{ Targeted cognitive function } & \multirow[b]{2}{*}{ Neuropsychological test } & \multicolumn{2}{|c|}{ Unadjusted M (SD) } & \multicolumn{2}{|c|}{ Adjusted M (SE) ${ }^{\mathrm{a}}$} & \multirow[b]{2}{*}{$F(d f 1, d f 2)$} & \multirow[b]{2}{*}{$p$} & \multirow[b]{2}{*}{$\eta_{p}^{2}$} \\
\hline & & $\mathrm{V}(n=130)$ & $\mathrm{NV}(n=708)$ & $\mathrm{V}(n=130)$ & $\mathrm{NV}(n=708)$ & & & \\
\hline \multicolumn{9}{|l|}{ Executive functions } \\
\hline \multirow[t]{4}{*}{ Inhibition } & $\mathrm{CPT}-\mathrm{HQ}^{\mathrm{b}}$ & $0.05(0.50)$ & $0.00(0.55)$ & $0.06(0.05)$ & $-0.01(0.02)$ & $1.38(1,739)$ & ns & .002 \\
\hline & Number of hits & $26.2(2.6)$ & $26.4(2.8)$ & & & & & \\
\hline & Mean reaction time hits ${ }^{c}$ & $44.2(7.9)$ & $42.8(8.7)$ & & & & & \\
\hline & Number of commission errors & $2.3(9.5)$ & $3.0(15.3)$ & & & & & \\
\hline \multirow[t]{3}{*}{ Cognitive flexibility } & $\mathrm{RST}^{\mathrm{b}}$ & $-0.04(0.80)$ & $0.01(0.75)$ & $-0.05(0.07)$ & $0.01(0.03)$ & $0.74(1,719)$ & ns & .001 \\
\hline & Accuracy cost & $23.3(23.8)$ & $22.2(23.6)$ & & & & & \\
\hline & Reaction time $\operatorname{cost}^{\mathrm{c}}$ & $26.1(18.9)$ & $25.3(18.9)$ & & & & & \\
\hline Fluid intelligence & WAIS-III Block Design subtest & $37.6(17.5)$ & $41.0(16.9)$ & $37.1(1.5)$ & $41.1(0.6)$ & $5.76(1,797)$ & .017 & .007 \\
\hline Planning & NAB Mazes Test ${ }^{d}$ & $15.3(6.7)$ & $17.0(6.3)$ & $15.0(0.7)$ & $17.1(0.3)$ & $7.32(1,468)$ & .007 & .015 \\
\hline \multicolumn{9}{|l|}{ Theory of mind } \\
\hline Affective & DFAR & 68.8 (9.9) & $68.9(10.7)$ & $68.7(1.0)$ & $68.9(0.4)$ & $0.03(1,717)$ & ns & $<.001$ \\
\hline Cognitive & Hinting Task & $17.0(2.9)$ & $17.7(2.7)$ & $17.0(0.2)$ & $17.7(0.1)$ & $6.09(1,789)$ & .014 & .008 \\
\hline
\end{tabular}

M, mean; SD, standard deviation; SE, standard error; V, violent; NV, nonviolent; df, degrees of freedom; CPT-HQ, Continuous Performance Test-HQ; ns, nonsignificant; RST, Response Shifting Task; WAIS-III, Wechsler Adult Intelligence Scale, Third Edition; NAB, Neuropsychological Assessment Battery; DFAR, Degraded Facial Affect Recognition Task.

Due to missing data, the total number of patients varies per test.

adjusted for age and sex.

Average of subscale $z$-scores.

c In centiseconds.

${ }^{\mathrm{d}}$ Administered six years after baseline. 
Table S4. Neuropsychological test performance in violent and nonviolent patients with psychotic disorders, excluding patients with PANSS total scores of 95 or higher.

\begin{tabular}{|c|c|c|c|c|c|c|c|c|}
\hline \multirow[b]{2}{*}{ Targeted cognitive function } & \multirow[b]{2}{*}{ Neuropsychological test } & \multicolumn{2}{|c|}{ Unadjusted M (SD) } & \multicolumn{2}{|c|}{ Adjusted M (SE) ${ }^{a}$} & \multirow[b]{2}{*}{$F(d f 1, d f 2)$} & \multirow[b]{2}{*}{$p$} & \multirow[b]{2}{*}{$\eta_{p}^{2}$} \\
\hline & & $\mathrm{V}(n=163)$ & $\mathrm{NV}(n=631)$ & $\mathrm{V}(n=163)$ & $\mathrm{NV}(n=631)$ & & & \\
\hline \multicolumn{9}{|l|}{ Executive functions } \\
\hline \multirow[t]{4}{*}{ Inhibition } & $\mathrm{CPT}-\mathrm{HQ}^{\mathrm{b}}$ & $0.00(0.54)$ & $0.00(0.56)$ & $0.01(0.05)$ & $-0.01(0.02)$ & $0.10(1,704)$ & ns & $<.001$ \\
\hline & Number of hits & $26.1(3.2)$ & $26.4(2.8)$ & & & & & \\
\hline & Mean reaction time hits ${ }^{c}$ & $43.7(8.0)$ & $42.8(8.7)$ & & & & & \\
\hline & Number of commission errors & $2.8(11.4)$ & $3.0(15.4)$ & & & & & \\
\hline \multirow[t]{3}{*}{ Cognitive flexibility } & $\mathrm{RST}^{\mathrm{b}}$ & $-0.05(0.78)$ & $0.02(0.74)$ & $-0.07(0.06)$ & $0.03(0.03)$ & $1.83(1,685)$ & ns & .003 \\
\hline & Accuracy cost & $23.1(24.5)$ & $21.7(23.1)$ & & & & & \\
\hline & Reaction time $\operatorname{cost}^{\mathrm{c}}$ & $27.1(19.1)$ & $25.4(18.6)$ & & & & & \\
\hline Fluid intelligence & WAIS-III Block Design subtest & $38.3(17.5)$ & $41.5(16.9)$ & $37.9(1.4)$ & $41.6(0.7)$ & $5.94(1,766)$ & .015 & .008 \\
\hline Planning & NAB Mazes Test ${ }^{d}$ & $15.9(6.6)$ & $16.9(6.3)$ & $15.5(0.6)$ & $17.0(0.3)$ & $4.87(1,466)$ & .028 & .010 \\
\hline \multicolumn{9}{|l|}{ Theory of mind } \\
\hline Affective & DFAR & 69.5 (9.7) & $69.0(10.5)$ & $69.4(0.9)$ & $69.0(0.4)$ & $0.11(1,693)$ & ns & $<.001$ \\
\hline Cognitive & Hinting Task & $17.0(3.0)$ & $17.8(2.7)$ & $17.0(0.2)$ & $17.8(0.1)$ & $8.85(1,758)$ & .003 & .012 \\
\hline
\end{tabular}

M, mean; SD, standard deviation; SE, standard error; V, violent; NV, nonviolent; df, degrees of freedom; CPT-HQ, Continuous Performance Test-HQ; ns, nonsignificant; RST, Response Shifting Task; WAIS-III, Wechsler Adult Intelligence Scale, Third Edition; NAB, Neuropsychological Assessment Battery; DFAR, Degraded Facial Affect Recognition Task.

Due to missing data, the total number of patients varies per test.

adjusted for age and sex.

${ }^{\mathrm{b}}$ Average of subscale $z$-scores.

c In centiseconds.

${ }^{\mathrm{d}}$ Administered six years after baseline. 


\section{Chapter 4}

Table S5. Neuropsychological test performance in violent and nonviolent patients with psychotic disorders, excluding patients with substance misuse.

\begin{tabular}{|c|c|c|c|c|c|c|c|c|}
\hline \multirow[b]{2}{*}{ Targeted cognitive function } & \multirow[b]{2}{*}{ Neuropsychological test } & \multicolumn{2}{|c|}{ Unadjusted M (SD) } & \multicolumn{2}{|c|}{ Adjusted M (SE) } & \multirow[b]{2}{*}{$F(d f 1, d f 2)$} & \multirow[b]{2}{*}{$p$} & \multirow[b]{2}{*}{$\eta_{p}^{2}$} \\
\hline & & $\mathrm{V}(n=55)$ & $\mathrm{NV}(n=274)$ & $\mathrm{V}(n=55)$ & $\mathrm{NV}(n=274)$ & & & \\
\hline \multicolumn{9}{|l|}{ Executive functions } \\
\hline \multirow[t]{4}{*}{ Inhibition } & $\mathrm{CPT}-\mathrm{HQ}^{\mathrm{b}}$ & $0.05(0.31)$ & $-0.01(0.65)$ & $0.06(0.09)$ & $-0.02(0.04)$ & $0.70(1,291)$ & ns & .002 \\
\hline & Number of hits & $26.2(2.3)$ & $26.4(2.7)$ & & & & & \\
\hline & Mean reaction time hits ${ }^{c}$ & $43.9(7.5)$ & $43.2(8.9)$ & & & & & \\
\hline & Number of commission errors & $1.3(2.0)$ & $3.9(20.3)$ & & & & & \\
\hline \multirow[t]{3}{*}{ Cognitive flexibility } & $\mathrm{RST}^{\mathrm{b}}$ & $0.02(0.72)$ & $0.03(0.79)$ & $0.01(0.11)$ & $0.03(0.05)$ & $0.03(1,287)$ & ns & $<.001$ \\
\hline & Accuracy cost & $25.2(25.7)$ & $23.2(24.5)$ & & & & & \\
\hline & Reaction time cost ${ }^{\mathrm{c}}$ & $21.9(15.0)$ & $24.1(19.2)$ & & & & & \\
\hline Fluid intelligence & WAIS-III Block Design subtest & $35.3(16.2)$ & $40.6(17.0)$ & $34.9(2.3)$ & $40.7(1.0)$ & $5.47(1,317)$ & .020 & .017 \\
\hline Planning & NAB Mazes Test ${ }^{d}$ & $14.0(6.7)$ & $16.6(6.4)$ & $13.7(1.1)$ & $16.6(0.5)$ & $6.20(1,190)$ & .014 & .032 \\
\hline \multicolumn{9}{|l|}{ Theory of mind } \\
\hline Affective & DFAR & $68.4(12.0)$ & $68.2(10.9)$ & $68.4(1.6)$ & $68.2(0.7)$ & $0.03(1,282)$ & ns & $<.001$ \\
\hline Cognitive & Hinting Task & $16.6(3.0)$ & $17.7(2.7)$ & $16.6(0.4)$ & $17.6(0.2)$ & $6.03(1,313)$ & .015 & .019 \\
\hline
\end{tabular}

M, mean; SD, standard deviation; SE, standard error; V, violent; NV, nonviolent; df, degrees of freedom; CPT-HQ, Continuous Performance Test-HQ; ns, nonsignificant; RST, Response Shifting Task; WAIS-III, Wechsler Adult Intelligence Scale, Third Edition; NAB, Neuropsychological Assessment Battery; DFAR, Degraded Facial Affect Recognition Task.

Due to missing data, the total number of patients varies per test.

adjusted for age and sex.

${ }^{\mathrm{b}}$ Average of subscale $z$-scores.

c In centiseconds.

${ }^{d}$ Administered six years after baseline. 
Table S6. Neuropsychological test performance in violent and nonviolent patients with psychotic disorders, including only patients with a secondary education.

\begin{tabular}{|c|c|c|c|c|c|c|c|c|}
\hline \multirow[b]{2}{*}{ Targeted cognitive function } & \multirow[b]{2}{*}{ Neuropsychological test } & \multicolumn{2}{|c|}{ Unadjusted M (SD) } & \multicolumn{2}{|c|}{ Adjusted M (SE) ${ }^{\mathrm{a}}$} & \multirow[b]{2}{*}{$F(d f 1, d f 2)$} & \multirow[b]{2}{*}{$p$} & \multirow[b]{2}{*}{$\eta_{p}^{2}$} \\
\hline & & $\mathrm{V}(n=146)$ & $\mathrm{NV}(n=620)$ & $\mathrm{V}(n=146)$ & $\mathrm{NV}(n=620)$ & & & \\
\hline \multicolumn{9}{|l|}{ Executive functions } \\
\hline \multirow[t]{4}{*}{ Inhibition } & $\mathrm{CPT}-\mathrm{HQ}^{\mathrm{b}}$ & $0.02(0.51)$ & $0.03(0.48)$ & $0.03(0.04)$ & $0.03(0.02)$ & $<0.01(1,674)$ & ns & $<.001$ \\
\hline & Number of hits & $26.2(2.6)$ & $26.5(2.6)$ & & & & & \\
\hline & Mean reaction time hits ${ }^{c}$ & $43.8(7.9)$ & $42.9(8.7)$ & & & & & \\
\hline & Number of commission errors & $2.7(11.7)$ & $2.4(11.1)$ & & & & & \\
\hline \multirow[t]{3}{*}{ Cognitive flexibility } & $\mathrm{RST}^{\mathrm{b}}$ & $0.02(0.70)$ & $0.00(0.76)$ & $0.00(0.07)$ & $0.00(0.03)$ & $<0.01(1,657)$ & ns & $<.001$ \\
\hline & Accuracy cost & $21.3(22.4)$ & $21.7(23.4)$ & & & & & \\
\hline & Reaction time $\operatorname{cost}^{\mathrm{c}}$ & $25.6(18.2)$ & $26.1(19.3)$ & & & & & \\
\hline Fluid intelligence & WAIS-III Block Design subtest & $39.8(17.7)$ & $42.3(16.4)$ & $39.4(1.4)$ & $42.3(0.7)$ & $3.44(1,727)$ & ns & .005 \\
\hline Planning & NAB Mazes Test ${ }^{d}$ & $16.2(6.4)$ & $17.1(6.2)$ & $15.8(0.6)$ & $17.2(0.3)$ & $4.14(1,473)$ & .042 & .009 \\
\hline \multicolumn{9}{|l|}{ Theory of mind } \\
\hline Affective & DFAR & $69.4(10.4)$ & $68.8(10.4)$ & $69.2(0.9)$ & $68.9(0.4)$ & $0.14(1,658)$ & ns & $<.001$ \\
\hline Cognitive & Hinting Task & $17.0(2.9)$ & $17.9(2.6)$ & $17.0(0.2)$ & $17.9(0.1)$ & $12.53(1,720)$ & $<.001$ & .017 \\
\hline
\end{tabular}

M, mean; SD, standard deviation; SE, standard error; V, violent; NV, nonviolent; df, degrees of freedom; CPT-HQ, Continuous Performance Test-HQ; ns, nonsignificant; RST, Response Shifting Task; WAIS-III, Wechsler Adult Intelligence Scale, Third Edition; NAB, Neuropsychological Assessment Battery; DFAR, Degraded Facial Affect Recognition Task.

Due to missing data, the total number of patients varies per test.

adjusted for age and sex.

${ }^{b}$ Average of subscale $z$-scores.

c In centiseconds.

${ }^{\mathrm{d}}$ Administered six years after baseline. 


\section{References}

1. Nuechterlein $\mathrm{KH}$, Barch DM, Gold JM, et al. Identification of separable cognitive factors in schizophrenia. Schizophr Res 2004; 72: 29-39.

2. Neuechterlein $\mathrm{KH}$, Dawson ME. Information processing and attentional functioning in the developmental course of schizophrenic disorders. Schizophr Bull 1984; 10: 160-203.

3. Bilder RM, Turkel E, Lipschutz-Broch L, et al. Antipsychotic medication effects on neuropsychological functions. Psychopharmacol Bull 1992; 28: 353-366.

4. Wechsler D. Wechsler Adult Intelligence Scale (3rd edition). Psychological Corporation, 1997.

5. Stern RA, White T. Neuropsychological Assessment Battery. Psychological Assessment Resources, 2003.

6. van 't Wout $M$, Aleman $A$, Kessels $R P$, et al. Emotional processing in a non-clinical psychosis-prone sample. Schizophr Res 2004; 68: 271-281.

7. van Dijke A, van 't Wout M, Ford JD, et al. Deficits in degraded facial affect labeling in schizophrenia and borderline personality disorder. PLoS One 2016; 11: e0154145.

8. Benton AL, Sivan AB, Hamsher KS. Benton's Test of Facial Recognition. Oxford University Press: New York, 1983.

9. Corcoran R, Mercer G, Frith CD. Schizophrenia, symptomatology and social inference: investigating "theory of mind" in people with schizophrenia. Schizophr Res 1995; 17: 513.

10. Brand N, Jolles J. Learning and retrieval rate of words presented auditorily and visually. J Gen Psychol 1985; 112: 201-210. 


\section{Chapter 5}

Risk factors for violence before and after the onset of schizophrenia spectrum disorder: a

Dutch nationwide case-control study

Lamsma J

Cahn W

Harte JM

GROUP investigators

Submitted for review 


\section{Abstract}

\section{Background}

It is unclear whether risk factors differ for violent behavior before (VBO) and after (VAO) the onset of schizophrenia spectrum disorder (SSD).

\section{Methods}

Data came from the Genetic Risk and Outcome of Psychosis project. The sample consisted of 886 patients with SSDs, recruited from diverse care settings in representative geographical areas of the Netherlands. Standardized instruments were used to measure environmental (parental deviance, childhood maltreatment, neighborhood disorganization) and clinical (substance misuse, impulsivity, delusions, hallucinations and lack of insight) risk factors for violent behavior. The timing of violence in relation to illness onset was ascertained from medical records and patient interviews. We compared patients with VBO, patients with VAO and nonviolent (NV) patients simultaneously on each risk factor with the $\chi^{2}$-test or analysis of variance. For post hoc analyses, Fisher's exact tests or Tukey-Kramer tests were used.

\section{Results}

Patients with VBO $\left(q_{730}=2.8, p=.015, d=0.5\right)$ and patients with VAO $\left(q_{812}=4.7, p<.001, d=\right.$ 0.5) were more impulsive than NV patients. Patients with VAO also had more often misused substances $\left(\chi^{2}{ }_{1}=6.6, p=.011, d=0.3\right)$ and poorer insight $\left(q_{813}=2.8, p=.015, d=0.3\right)$ than NV patients. Delusions were more severe in patients with VAO than in patients with VBO $\left(q_{169}\right.$ $=2.1, p=.088, d=0.3)$ and NV patients $\left(q_{808}=2.9, p=.011, d=0.3\right)$.

\section{Conclusion}

Strategies to predict and prevent violence in SSD should distinguish between VBO and VAO. 
Risk factors for violence before and after the onset of schizophrenia spectrum disorder

\section{Introduction}

It is well established that schizophrenia spectrum disorder (SSD) increases the risk of violent behavior $^{1,2}$. In a meta-analysis, the odds of violence in cases were about five times those of unaffected controls ${ }^{1}$. Estimates of the population attributable fraction are as high as $10 \%{ }^{3}$.

Among the strongest risk factors for violence in SSD are parental deviance, childhood maltreatment, substance misuse, impulsivity and lack of insight ${ }^{4}$. Regarding psychotic symptoms, both delusions $s^{5,6}$ and hallucinations ${ }^{7,8}$ have shown associations with violent behavior. Despite having received little attention, neighborhood disorganization may be important as well ${ }^{9}$. Apart from psychotic symptoms and poor insight, all of these are risk factors for violence in the general population ${ }^{10}$.

Risk factors may differ depending on whether violent behavior develops before (VBO) or after (VAO) the onset of SSD. However, the available evidence is unclear. In a register-based study of all schizophrenia patients born in Denmark between 1963 and 1989 ( $N=4179)$, substance misuse at initial treatment contact almost quadrupled (hazard ratio 3.7, 95\% confidence interval 2.5-5.4) the likelihood of subsequently being convicted of a violent crime for the first time ${ }^{11}$. Other studies have consistently reported higher rates of substance misuse in patients with conduct disorder in their youth than in those without ${ }^{12-15}$ and in patients who had committed any crime before becoming ill than in those who had only done so afterwards ${ }^{16-21}$. Their results for parental deviance ${ }^{14,16,18}$, childhood maltreatment ${ }^{12,14,21}$ and psychotic symptoms ${ }^{18,21,22}$ are conflicting. Furthermore, they have been limited by crude definitions of illness onset (e.g. age of 18, first psychiatric admission) and forensic samples.

To address this uncertainty, we have investigated risk factors for VBO and VAO using a sensitive outcome measure in a nationwide sample of individuals with SSDs. We hypothesized that risk factors for VBO are the same as for the general population and that risk factors for VAO are illness related. 


\section{Methods}

\section{Setting and participants}

Data were collected as part of a larger research project, called Genetic Risk and Outcome of Psychosis (GROUP). GROUP is conducted by 4 university hospitals and 36 affiliated mental healthcare centers in the Netherlands. These institutions are located in representative geographical areas of the country and provide access to treatment in a variety of settings to approximately $75 \%$ of the population. Throughout 2004 , consecutive patients were invited to participate if they met the following criteria: (i) age between 16 and 50; (ii) good command of the Dutch language; (iii) Diagnostic and Statistical Manual of Mental Disorders, Fourth Edition, Text Revision (DSM-IV-TR) ${ }^{23}$ diagnosis of schizophrenia or other nonaffective psychotic disorder; and (iv) able and willing to give written informed consent. Parents were also recruited. To them, inclusion criteria ii and iv applied. Participants were followed up twice every three years. Assessments took place at the university hospitals.

\section{Measures}

We used baseline data, unless stated otherwise. The instruments' psychometric properties have been described elsewhere ${ }^{24}$.

\section{Risk factors for violent behavior}

The Comprehensive Assessment of Symptoms and History ${ }^{25}$ was used at three of the four university hospitals to determine whether parents had ever been aggressive. It defines aggression as the expression of anger that is grossly inappropriate or disproportionate to the situation at hand. 
Risk factors for violence before and after the onset of schizophrenia spectrum disorder

Histories of substance misuse in parents and patients were established with the Substance Abuse Module of the Composite International Diagnostic Interview ${ }^{26}$. We defined alcohol misuse as a regular intake of $>50$ units per week for men and $>35$ units per week for women. These cut-offs represent 'high-risk drinking' in the UK's National Institute for Health and Clinical Excellence ${ }^{27}$ guidelines. For other substances, misuse referred to a DSM-IV-TR diagnosis of abuse or dependence.

At the second wave, the Childhood Trauma Questionnaire-Short Form ${ }^{28}$ was used to assess childhood experiences of neglect and abuse in patients. It consists of 25 items, rated on a 5point scale. Anchors are 'never true' (1) and 'very often true' (5).

Social disorganization in the patient's current neighborhood was measured at the third wave with the Social Environment Assessment Tool ${ }^{29}$. There are seven items, covering: frequency and fear of crime, informal social control, social cohesion, ethnic diversity, physical conditions and community involvement. Response options vary between 1 (e.g. 'almost never', 'very unlikely') and 5 (e.g. 'almost always', 'very likely').

The Positive and Negative Syndrome Scale ${ }^{30}$ was used to measure impulsivity, delusions, hallucinations and insight in patients over the previous week. For delusions, we aggregated items P1 ('delusions'), P5 ('grandiosity') and P6 ('suspiciousness'). Items are scored from 1 ('no impairment') to 7 ('severe impairment').

VBO and VAO

The Life Chart Schedule ${ }^{31}$ recorded whether the patient had physically attacked someone before their first psychotic episode or thereafter. This information was extracted from medical records and interviews with the patient and, where possible, their parents. 


\section{Statistical analysis}

We simultaneously compared patients with VBO, patients with VAO and nonviolent (NV) patients on each risk factor with the $\chi^{2}$-test or analysis of variance (ANOVA). The level of statistical significance was set at $5 \%$. After a significant $\chi^{2}$-test, we computed exact $p$-values for the pairwise comparisons ${ }^{32}$. Multiplicity corrections were made with the Simes procedure ${ }^{33}$. To improve the validity of the $\chi^{2}$-tests, we required at least five observations per cell in the corresponding contingency tables ${ }^{34}$. Since one of the cell counts was lower for parental aggression, we combined this variable with parental substance misuse as 'parental deviance'. TukeyKramer tests were used for post hoc analyses of ANOVAs. On theoretical grounds, we adjusted significant pairwise comparisons for the potential confounders sex and age. This was done in logistic or linear regression models with group membership as an independent variable. Cohen's $d$ served as a measure of effect size. Odds ratios and regression coefficients were converted to Cohen's $d$ with the formulas in Lipsey and Wilson ${ }^{35}$. Analyses were carried out in SPSS 21.

\section{Results}

Table 1 shows the patients' $(N=886)$ demographic and clinical characteristics at baseline. Over half the patients were male and had received a diagnosis of schizophrenia $(n=487$, 55\%). There were $48(5 \%)$ patients with VBO and 130 (15\%) patients with VAO. On average, these patients were younger and had more psychiatric admissions than NV patients.

Group differences in risk factors for violent behavior are presented in Table 2. Significant main effects were found for impulsivity $\left(F_{2,856}=13.8, p<.001\right)$, substance misuse $\left(\chi^{2}{ }_{2}=6.9, p=\right.$ $.031)$, delusions $\left(F_{2,851}=4.6, p=.011\right)$ and lack of insight $\left(F_{2,856}=4.0, p=.019\right)$. Post hoc analyses revealed that patients with VBO $\left(q_{730}=2.8, p=.015, d=0.5\right)$ and patients with VAO $\left(q_{812}=4.7, p<.001, d=0.5\right)$ were significantly more impulsive than NV patients. Patients with 
Table 1. Sample characteristics at baseline.

\begin{tabular}{|c|c|c|c|c|c|c|c|}
\hline \multirow[b]{2}{*}{ Characteristic } & \multirow[b]{2}{*}{$\mathrm{NV}(n=708)$} & \multirow[b]{2}{*}{$\operatorname{VBO}(n=48)$} & \multirow[b]{2}{*}{$\operatorname{VAO}(n=130)$} & \multirow[b]{2}{*}{ Main effect } & \multicolumn{3}{|c|}{ Pairwise comparisons } \\
\hline & & & & & NV vs VBO & NV vs VAO & VBO vs VAO \\
\hline \multicolumn{8}{|l|}{ Demographic characteristics } \\
\hline Male, $n(\%)$ & $543(77)$ & $34(71)$ & $108(83)$ & $\begin{array}{l}\chi^{2}{ }_{2}=3.8 \\
p=.152\end{array}$ & & & \\
\hline Age, M (SD) & $27.6(7.2)$ & $25.6(6.1)$ & $26.0(6.2)$ & $\begin{array}{c}F_{2,883}=4.4 \\
p=.013\end{array}$ & $\begin{array}{c}q_{754}=1.9 \\
p=.135\end{array}$ & $\begin{array}{c}q_{836}=2.4 \\
p=.040\end{array}$ & $\begin{array}{c}q_{176}=0.3 \\
p=.947\end{array}$ \\
\hline White, $n(\%)$ & $559(80)$ & $38(79)$ & $96(75)$ & $\begin{array}{l}\chi^{2}{ }_{2}=1.3 \\
p=.515\end{array}$ & & & \\
\hline \multicolumn{8}{|l|}{ Clinical characteristics } \\
\hline Schizophrenia, $n(\%)$ & $479(68)$ & $38(79)$ & $95(73)$ & $\begin{array}{l}\chi^{2}{ }^{2}=3.9 \\
p=.140\end{array}$ & & & \\
\hline Age of onset, M (SD) & $23.4(6.6)$ & $21.8(6.5)$ & $21.6(5.3)$ & $\begin{array}{c}F_{2,883}=5.5 \\
p=.004\end{array}$ & $\begin{array}{c}q_{754}=1.8 \\
p=.180\end{array}$ & $\begin{array}{c}q_{836}=3.0 \\
p=.008\end{array}$ & $\begin{array}{c}q_{176}=0.1 \\
p=.992\end{array}$ \\
\hline Number of inpatient stays ${ }^{a}, M(S D)$ & $1.7(1.8)$ & $2.2(1.9)$ & $2.3(1.8)$ & $\begin{array}{c}F_{2,789}=7.4 \\
p=.001\end{array}$ & $\begin{array}{c}q_{667}=1.8 \\
p=.167\end{array}$ & $\begin{array}{c}q_{745}=3.6 \\
p=.001\end{array}$ & $\begin{array}{c}q_{166}=0.4 \\
p=.905\end{array}$ \\
\hline Violent behavior & & & & & & & \\
\hline Number of incidents ${ }^{a}, M(S D)$ & $2.6(2.5)$ & $2.1(2.1)$ & & & & & $\begin{array}{c}t_{166}=1.3 \\
p=.206\end{array}$ \\
\hline Injured victim, $n(\%)$ & $12(27)$ & $42(33)$ & & & & & $\begin{array}{l}\chi^{2}{ }_{1}=0.6 \\
p=.426\end{array}$ \\
\hline
\end{tabular}

$\mathrm{NV}$, no violence; $\mathrm{VBO}$, violence before illness onset; $\mathrm{VAO}$, violence after illness onset.

Statistically significant results are in bold.

a Outliers were capped at 10. 


\section{Chapter 5}

Table 2. Risk factors for violence before and after illness onset in patients with schizophrenia spectrum disorders.

Pairwise comparisons

\begin{tabular}{|c|c|c|c|c|c|c|c|}
\hline \multirow[b]{2}{*}{ Risk factor } & \multirow[b]{2}{*}{$\mathrm{NV}(n=708)$} & \multirow[b]{2}{*}{ VBO $(n=48)$} & \multirow[b]{2}{*}{$\operatorname{VAO}(n=130)$} & \multirow[b]{2}{*}{ Main effect } & \\
\hline & & & & & NV vs VBO & NV vs VAO & VBO vs VAO \\
\hline \multicolumn{8}{|l|}{ Environmental risk factors } \\
\hline Parental deviance, $n(\%)$ & $69(18)$ & $6(21)$ & $15(20)$ & $\begin{array}{l}\chi^{2}{ }_{2}=0.4 \\
p=.814\end{array}$ & & & \\
\hline Childhood maltreatment, M (SD) & $39.8(12.0)$ & $41.4(15.7)$ & $41.1(12.5)$ & $\begin{array}{c}F_{2,612}=0.6 \\
p=.560\end{array}$ & & & \\
\hline Neighborhood disorganization & $19.8(3.2)$ & $20.7(3.2)$ & $20.6(3.7)$ & $\begin{array}{c}F_{2,387}=1.9 \\
p=.146\end{array}$ & & & \\
\hline \multicolumn{8}{|l|}{ Clinical risk factors } \\
\hline Substance misuse, $n(\%)$ & 330 (49) & $25(56)$ & $76(62)$ & $\begin{array}{l}\chi^{2} 2=6.9 \\
p=.031\end{array}$ & $\begin{array}{c}\chi^{2}{ }_{1}=0.7 \\
p=.444 \\
d=0.1\end{array}$ & $\begin{array}{c}\chi^{2}{ }_{1}=6.6 \\
p=.011 \\
d=0.3\end{array}$ & $\begin{array}{c}\chi^{2}{ }_{1}=0.5 \\
p=.482 \\
d=0.1\end{array}$ \\
\hline Impulsivity, M (SD) & $1.2(0.6)$ & $1.6(0.9)$ & $1.6(1.0)$ & $\begin{array}{c}F_{2,856}=13.8 \\
p<.001\end{array}$ & $\begin{array}{c}q_{730}=2.8 \\
p=.015 \\
d=0.5\end{array}$ & $\begin{array}{c}q_{812}=4.7 \\
p<.001 \\
d=0.5\end{array}$ & $\begin{array}{c}q_{170}=0.2 \\
p=.987 \\
d<0.1\end{array}$ \\
\hline Psychotic symptoms & & & & & & & \\
\hline Delusions, M (SD) & $6.1(3.1)$ & $5.9(3.1)$ & $7.0(3.4)$ & $\begin{array}{c}F_{2,851}=4.6 \\
p=.011\end{array}$ & $\begin{array}{c}q_{725}=0.6 \\
p=.833 \\
d=0.1\end{array}$ & $\begin{array}{c}q_{808}=2.9 \\
p=.011 \\
d=0.3\end{array}$ & $\begin{array}{c}q_{169}=2.1 \\
p=.088 \\
d=0.3\end{array}$ \\
\hline Hallucinations, M (SD) & $2.1(1.5)$ & $1.9(1.4)$ & $2.3(1.6)$ & $\begin{array}{c}F_{2,857}=1.4 \\
p=.252\end{array}$ & & & \\
\hline Poor insight, M (SD) & $2.1(1.4)$ & $2.2(1.4)$ & $2.4(1.5)$ & $\begin{array}{c}F_{2,856}=4.0 \\
p=.019\end{array}$ & $\begin{array}{c}q_{729}=0.7 \\
p=.768 \\
d=0.1\end{array}$ & $\begin{array}{c}q_{813}=2.8 \\
p=.015 \\
d=0.3\end{array}$ & $\begin{array}{c}q_{170}=0.9 \\
p=.627 \\
d=0.2\end{array}$ \\
\hline
\end{tabular}

NV, no violence; VBO, violence before illness onset; VAO, violence after illness onset.

Statistically significant results are in bold. 
Risk factors for violence before and after the onset of schizophrenia spectrum disorder

VAO also had significantly more often misused substances $\left(\chi^{2}{ }_{1}=6.6, p=.011, d=0.3\right)$, more severe delusions $\left(q_{808}=2.9, p=.011, d=0.3\right)$ and poorer insight $\left(q_{813}=2.8, p=.015, d=0.3\right)$ than NV patients. Delusions in patients with VAO were nonsignificantly, but moderately, more severe than in patients with VBO $\left(q_{169}=2.1, p=.088, d=0.3\right)$. Adjustments for sex and age did not materially change the results (Table 3 ).

\section{Discussion}

In a nationwide sample of 886 patients with schizophrenia spectrum disorders (SSDs), we have investigated risk factors for violent behavior before (VBO) and after (VAO) illness onset. We found that patients with VBO and patients with VAO were more impulsive than nonviolent (NV) patients. Patients with VAO also had more often misused substances and poorer insight than NV patients. Lastly, delusions in patients with VAO were more severe than in patients with VBO and NV patients.

These findings suggest that both VBO (contrary to our hypothesis) and VAO (consistent with our hypothesis) are mainly illness related. Impulsivity is a clinical feature of SSD ${ }^{36}$. Moreover, relevant to VBO, it may present before the onset of illness. This is supported by two lines of evidence. First, conduct disorder ${ }^{37}$ and attention deficit hyperactivity disorder ${ }^{38}-$ mental disorders characterized by impulsivity - are precursors of SSD. Second, common brain abnormalities in SSD, such as low-grade inflammation, hypofunction of $\mathrm{N}$-methyl-D-aspartate receptors and disruption of frontostriatal circuits 39,40 , have been linked to impulsive behavior in the general population ${ }^{41-43}$ and precede illness onset by many years ${ }^{39}$. Impulsivity predisposes a person to violence by lowering their concern for its consequences ${ }^{44}$. Substance misuse is a strong predictor of treatment nonadherence ${ }^{45}$. Some substances, notably cannabis, amphetamines and hallucinogens may cause or worsen delusions after prolonged use ${ }^{46}$. Delusions may motivate violent behavior, especially when they induce anger ${ }^{6}$. Poor insight makes it more likely that someone will act on delusions ${ }^{47}$ or refuse treatment ${ }^{45}$. 
Chapter 5

Table 3. Regression weights for pairwise comparisons that were statistically significant in post hoc analyses, adjusted for sex and age.

\begin{tabular}{llccc}
\hline Risk factor & Comparison & $b(\mathrm{SE})$ & $p$ & $d$ \\
\hline Substance misuse & NV vs VAO & $0.4(0.2)$ & .034 & 0.2 \\
Impulsivity & NV v VBO & $0.3(0.1)$ & .003 & 0.5 \\
Impulsivity & NV vs VAO & $0.3(0.1)$ & $<.001$ & 0.5 \\
Delusions & NV vs VAO & $0.8(0.3)$ & .006 & 0.3 \\
Poor insight & NV vs VAO & $0.3(0.1)$ & .010 & 0.2 \\
\hline
\end{tabular}

$\mathrm{NV}$, no violence; VBO, violence before illness onset; $\mathrm{VAO}$, violence after illness onset. 
Risk factors for violence before and after the onset of schizophrenia spectrum disorder

This study benefits from a number of strengths. First, patients were recruited from diverse geographical areas and care settings, making the results widely generalizable. Second, sampling was independent of the variables of interest. This partially prevented selection bias. Finally, the use of multiple data sources increased the sensitivity of the outcome measure. However, there are several limitations. First, the case-control design precludes causal inference. In addition, time lag and treatment with antipsychotics may have attenuated associations for impulsivity, delusions and hallucinations ${ }^{48}$. Neighborhood disorganization and substance misuse may have been consequences of SSD. However, evidence for the social drift ${ }^{49}$ and selfmedication ${ }^{50}$ theories is weak. Second, we only controlled for two potential confounders (sex and age). Others were not measured or lay on the causal pathway to violence ${ }^{51}$. Third, missing data may have introduced bias. We decided against multiple imputation, as missingness was either negligible $(<5 \%)$ or substantial $(30 \%-55 \%)$ and possibly dependent on the exposure $\mathrm{e}^{52}$. Fourth, statistical power was low for a few post hoc analyses of VBO. We therefore gave effect sizes precedence over $p$-values in the interpretation of their results ${ }^{53}$. Finally, the age at which patients became violent was not recorded. It has been proposed that VBO typically begins in childhood and continues across the lifespan, while VAO is transient ${ }^{54}$. However, we found no difference in frequency between VBO and VAO.

A clinical implication of the findings is that a distinction should be made between VBO and VAO in risk assessment and management. Whether this improves the performance of standardized tools will have to be determined ${ }^{55}$. To clarify causal mechanisms, studies with prospective designs and larger samples are needed. 


\section{References}

1. Fazel S, Gulati G, Linsell L, et al. Schizophrenia and violence: systematic review and metaanalysis. PloS Med 2009; 6: e100012.

2. Large MM, Nielssen O. Violence in first-episode psychosis: a systematic review and metaanalysis. Schizophr Res 2011; 125: 209-220.

3. Fazel S, Smith EN, Chang Z, et al. Risk Factors for interpersonal violence: an umbrella review of meta-analyses. Br J Psychiatry 2018; 213: 609-614.

4. Witt K, Van Dorn RA, Fazel S. Risk factors for violence in psychosis: systematic review and meta-regression analysis of 110 studies. PLoS One 2013; 8: e55942.

5. Coid JW, Ullrich S, Kallis C, et al. The relationship between delusions and violence: findings from the East London First Episode Psychosis Study. JAMA Psychiatry 2013; 70: 465-471.

6. Ullrich S, Keers R, Coid JW. Delusions, anger, and serious violence: new findings from the MacArthur Violence Risk Assessment Study. Schizophr Bull 2014; 40: 1174-1178.

7. Haddock G, Eisner E, Davies G, et al. Psychotic symptoms, self-harm and violence in individuals with schizophrenia and substance misuse problems. Schizophr Res 2013; 151: 215-220.

8. Swanson JW, Swartz MS, Van Dorn RA, et al. A national study of violent behavior in persons with schizophrenia. Arch Gen Psychiatry 2006; 63: 490-499.

9. Markowitz FE. Mental illness, crime, and violence: risk, context, and social control. Aggress Violent Behav 2011; 16: 36-44.

10. Farrington DP, Gaffney H, Ttofi MM. Systematic reviews of explanatory risk factors for violence, offending, and delinquency. Aggress Violent Behav 2017; 33: 24-36.

11. Munkner R, Haastrup S, Joergensen T. Incipient offending among schizophrenia patients after first contact to the psychiatric hospital system. Eur Psychiatry 2005; 20: 321-326.

12. Mathieu C, Côté G. A modelization of differences between early- and late-starter FrenchCanadian offenders. Int J Forensic Ment Health 2009; 8: 25-32.

13. Moran P, Hodgins S. The correlates of comorbid antisocial personality disorder in schizophrenia. Schizophr Bull 2004; 30: 791-802. 
Risk factors for violence before and after the onset of schizophrenia spectrum disorder

14. Sánchez-SanSegundo M, Ferrer-Cascales R, Herranz-Bellido J, et al. Subtypes of severely mentally ill violent offenders in a Spanish forensic psychiatric hospital. Int J Forensic Ment Health 2014; 13: 217-226.

15. Swanson JW, Van Dorn RA, Swartz MS, et al. Alternative pathways to violence in persons with schizophrenia: the role of childhood antisocial behavior problems. Law Hum Behav 2008; 32: 228-224.

16. Jones RM, Van den Bree $M$, Ferriter $M$, et al. Childhood risk factors for offending before first psychiatric admission for people with schizophrenia: a case-control study of high security hospital admissions. Behav Sci Law 2010; 28: 351-356.

17. Kooyman I, Walsh E, Stevens $H$, et al. Criminal offending before and after the onset of psychosis: examination of an offender typology. Schizophr Res 2012; 140: 198-203.

18. Laajasalo $T$, Häkkänen $H$. Offence and offender characteristics among two groups of Finnish homicide offenders with schizophrenia: comparison of early-and late-start offenders. J Forensic Psychiatry Psychol 2005; 16: 41-59.

19. Simpson Al, Grimbos T, Chan C, et al. Developmental typologies of serious mental illness and violence: evidence from a forensic psychiatric setting. Aust N Z J Psychiatry 2015; 49: 1048-1055.

20. Tengström A, Hodgins S, Kullgren G. Men with schizophrenia who behave violently: the usefulness of an early- versus late-start offender typology. Schizophr Bull 2001; 27: 205218.

21. van Dongen JD, Buck NM, Barendregt M, et al. Anti-social personality characteristics and psychotic symptoms: two pathways associated with offending in schizophrenia. Crim Behav Ment Health 2015; 25: 181-191.

22. Munkner R, Haastrup S, Joergensen T, et al. The association between psychopathology of first-episode psychosis patients within the schizophrenia spectrum and previous offending. Nord J Psychiatry 2009; 63: 124-131.

23. American Psychiatric Association. Diagnostic and Statistical Manual of Mental Disorders (4th edition, text revision). Author, 2000.

24. Korver N, Quee PJ, Boos HB, et al. Genetic Risk and Outcome of Psychosis (GROUP), a multi-site longitudinal cohort study focused on gene-environment interaction: 
objectives, sample characteristics, recruitment and assessment methods. Int J Methods Psychiatr Res 2012; 21: 205-221.

25. Andreasen NC, Flaum M, Arndt S. The Comprehensive Assessment of Symptoms and History (CASH). An instrument for assessing diagnosis and psychopathology. Arch Gen Psychiatry 1992; 49: 615-623.

26. World Health Organization. Composite International Diagnostic Interview. WHO, 1990.

27. The National Institute for Health and Clinical Excellence. Alcohol-use disorders: prevention. NICE, 2010.

28. Bernstein DP, Stein JA, Newcomb MD, et al. Development and validation of a brief screening version of the Childhood Trauma Questionnaire. Child Abuse Negl 2003; 27: 169-190.

29. Kirkbride JB. Social Environment Assessment Tool (unpublished manuscript). University College London, 2016.

30. Kay SR, Fiszbein A, Opler LA. The positive and negative syndrome scale (PANSS) for schizophrenia. Schizophr Bull 1987; 13: 261-276.

31. Susser E, Finnerty M, Mojtabai R, et al. Reliability of the life chart schedule for assessment of the long-term course of schizophrenia. Schizophr Res 2000; 42: 67-77.

32. Shan G, Gerstenberger S. Fisher's exact approach for post hoc analysis of a chi-squared test. PLoS One 2017; 12: e0188709.

33. Simes RJ. An improved Bonferroni procedure for multiple tests of significance. Biometrika 1986; 73: 751-754.

34. Kraska-Miller M. Nonparametric statistics for social and behavioral sciences. CRC Press, 2014.

35. Lipsey MW, Wilson DB. Practical meta-analysis. SAGE Publishing, 2001.

36. Ouzir M. Impulsivity in schizophrenia: a comprehensive update. Aggress Violent Behav 2013; 18: 247-254.

37. Hodgins S. Violent behaviour among people with schizophrenia: a framework for investigations of causes, and effective treatment, and prevention. Philos Trans R Soc Lond B Biol Sci 2008; 363: 2505-2518. 
Risk factors for violence before and after the onset of schizophrenia spectrum disorder

38. Dalsgaard S, Mortensen PB, Frydenberg M, et al. Association between attention-deficit hyperactivity disorder in childhood and schizophrenia later in adulthood. Eur Psychiatry 2014; 29: 259-263.

39. Kahn RS, Sommer IE. The neurobiology and treatment of first-episode schizophrenia. Mol Psychiatry 2015; 20: 84-89.

40. Kelly S, Jahanshad N, Zalesky A, et al. Widespread white matter microstructural differences in schizophrenia across 4322 individuals: results from the ENIGMA Schizophrenia DTI Working Group. Mol Psychiatry 2018; 23: 1261-1269.

41. Gassen J, Prokosch ML, Eimerbrink MJ, et al. Inflammation predicts decision-making characterized by impulsivity, present focus, and an inability to delay gratification. Sci Rep 2019; 9: 4928.

42. Fineberg NA, Chamberlain SR, Goudriaan AE, et al. New developments in human neurocognition: clinical, genetic, and brain imaging correlates of impulsivity and compulsivity. CNS Spectr 2014; 19: 69-89.

43. Pattij T, Vanderschuren $\sqcup$. The neuropharmacology of impulsive behaviour. Trends Pharmacol Sci 2008; 29: 192-199.

44. Moeller FG, Barratt ES, Dougherty DM, et al. Psychiatric aspects of impulsivity. Am J Psychiatry 2001; 158: 1783-1793.

45. Semahegn A, Torpey K, Manu A, et al. Psychotropic medication non-adherence and its associated factors among patients with major psychiatric disorders: a systematic review and meta-analysis. Syst Rev 2018; 7: 10.

46. Paparelli A, Di Forti M, Morrison PD, et al. Drug-induced psychosis: how to avoid star gazing in schizophrenia research by looking at more obvious sources of light. Front Behav Neurosci 2011; 5: 1 .

47. Bjørkly S. Empirical evidence of a relationship between insight and risk of violence in the mentally ill: a review of the literature. Aggress Violent Behav 2006; 11: 414-423.

48. Morrissette DA, Stahl SM. Modulating the serotonin system in the treatment of major depressive disorder. CNS Spectr 2014; 19 Suppl 1: 57-67.

49. Sariaslan A, Fazel S, D'Onofrio BM, et al. Schizophrenia and subsequent neighborhood deprivation: revisiting the social drift hypothesis using population, twin and molecular genetic data. Translational Psychiatry 2016; 6: e796. 
Chapter 5

50. Lembke A. Time to abandon the self-medication hypothesis in patients with psychiatric disorders. Am J Drug Alcohol Abuse 2012; 38: 524-529.

51. Cole SR, Hernán MA. Fallibility in estimating direct effects. Int J Epidemiol 2002; 31: 163165.

52. Hughes RA, Heron J, Sterne JA. Accounting for missing data in statistical analyses: multiple imputation is not always the answer. Int J Epidemiol 2019; 48: 1294-1304.

53. Sullivan GM, Feinn R. Using effect size - or why the p-value is not enough. J Grad Med Educ 2012; 4: 279-282.

54. Hodgins S, Klein S. New clinically relevant findings about violence by people with schizophrenia. Can J Psychiatr 2017; 62: 86-93.

55. Singh JP, Serper $M$, Reinharth J, et al. Structured assessment of violence risk in schizophrenia and other psychiatric disorders: a systematic review of the validity, reliability, and item content of 10 available instruments. Schizophr Bull 2011; 37: 899912. 


\section{Chapter 6}

Structural brain correlates of interpersonal violence: systematic review and voxel-based meta-analysis of neuroimaging studies

Lamsma J

Mackay C

Fazel S

Psychiatry Res Neuroimaging 2017; 30: 69-73. 
Chapter 6

\section{Abstract}

Owing to inconsistent nomenclature and results, we have undertaken a label-based review and anatomical likelihood estimation (ALE) meta-analysis of studies measuring the quantitative association between regional grey matter (GM) volume and interpersonal violence. Following PRISMA guidelines, we identified studies by searching 3 online databases (Embase, Medline, Psyclnfo) and reference lists. Thirty-five studies were included in the label-based review, providing information for 1,288 participants and 86 brain regions. Per region, $0-57 \%$ of the results indicated significant reductions in GM volume, while 0-23\% indicated significant increases. The only region for which more than half of all results indicated significant reductions was the parietal lobe. However, these results were dispersed across subregions. The ALE meta-analysis, which included 6 whole-brain voxel-based morphometry studies totaling 278 participants and reporting 144 foci, showed no significant clusters of reduced GM volume. No material differences were observed when excluding experiments using reactive violence as outcome or subjects diagnosed with psychopathy. Possible explanations for these findings are phenomenological and etiological heterogeneity and insufficient power in the label-based review and ALE meta-analysis to detect small effects. We recommend that future studies distinguish between subtypes of interpersonal violence and investigate mediation by underlying emotional and cognitive processes. 


\section{Introduction}

Over the last two decades, there have been numerous structural neuroimaging studies of interpersonal violence. However, the large number of different brain regions reported, variation in nomenclature and conflicting results have made interpretation difficult. Previous reviews have been nonsystematic ${ }^{1}$ or limited to small numbers of selected brain regions ${ }^{2}$. It has also been common practice in reviews to conflate measures of violent behavior with indirect measures such as personality traits (e.g. poor impulse control, hostility) and psychiatric diagnoses (e.g. antisocial personality disorder [APD], psychopathy) ${ }^{3}$.

Understanding the neurobiological correlates of interpersonal violence is important for the development of: (i) interventions to prevent and reduce violence; (ii) methods for screening and targeting individuals at risk for violence; (iii) risk assessment tools informing involuntary admission, sentencing and release decisions; and (iv) evaluation in criminal cases concerning the degree of a defendant's culpability and risk of future violence.

Therefore, we present a systematic label-based review ${ }^{4}$ of neuroimaging studies investigating the quantitative association between regional grey matter (GM) volume and interpersonal violence. We also performed an anatomical likelihood estimation (ALE) meta-analysis of voxel-based morphometry (VBM) studies examining volumetric reductions in regional GM.

\section{Methods}

This review was conducted in accordance with the Preferred Reporting Items for Systematic Reviews and Meta-Analyses guidelines ${ }^{5}$. 


\section{Search strategy}

We searched for studies indexed in the online databases Embase, Medline and Psyclnfo from January 1990 to December 2014. Keywords were inclusive for violent behavior ("violen*", "aggressi*", "prison*", "crim*", "offen*"), structural neuroimaging techniques ("neuroimaging", "brain imaging", "computed tomography", "CT", "magnetic resonance imaging", "MRI", "diffusion tensor imaging", "DTI") and - to make the search more focused - brain regions that have usually received the most attention in neuroimaging studies of violent behavior (i.e. "amygdala", "prefrontal cortex", "temporal cortex"). Additional studies were found by manually scanning the references of included studies and a number of recent review articles ${ }^{6-10}$. Finally, we searched citations to included studies indexed in Google Scholar Citations from January 1990 to December 2014. Our search included grey literature (e.g. dissertations, conference papers, working papers). Authors were contacted if studies were unobtainable or additional information was required.

\section{Study selection}

Studies were eligible when meeting the following criteria: (i) the study contained primary data; (ii) the study was available in the English language; (iii) the study was conducted in or after 1990; (iv) independent samples contained 10 or more participants; (v) all participants were aged 18 or older; (vi) the study used in vivo neuroimaging by means of computed tomography or structural magnetic resonance imaging (including diffusion tensor imaging $[D T I])$; and (vii) the study measured the quantitative association between violent behavior and at least one structural parameter (e.g. volume, fractional anisotropy) using betweengroup, correlation or regression analysis. We defined violent behavior as the intentional use of physical force to harm another person. To be included in the label-based review, the study had to provide sufficient information to code a result for at least one discrete brain region as negative, positive or nonsignificant. A negative result indicated a statistically significant association between violence and a reduction in GM volume, while a positive result indicated a 
statistically significant association between violence and an increase in GM volume. For ALE meta-analysis, we considered studies that conducted whole brain voxel-based analyses and reported coordinates for at least one peak voxel in either Montreal Neurological Institute or Talairach space.

We excluded: (i) samples that consisted of pedophilic offenders or participants with some form of brain lesion or malformation (e.g. cavum septum pellucidum), intellectual disability, epilepsy or a neurodegenerative disease (e.g. Huntington's disease, Alzheimer's disease); (ii) analyses comparing qualitatively different types of violence (e.g. homicide vs rape); (iii) psychiatric diagnoses and personality traits that are not defined by the actual display of violent behavior (e.g. psychopathy, impulsivity); and (iv) instruments primarily designed to assess a person's inclination toward violent behavior (e.g. Buss-Perry Aggression Questionnaire, StateTrait Anger Expression Inventory).

\section{Data extraction}

For any combination of structural parameter and tissue class, we required a minimum of 5 experiments per: (i) brain region for label-based reviews; and (ii) contrast of interest (i.e. reduction, increase) for ALE meta-analyses. Studies examining indices of white matter (WM) integrity (i.e. fractional anisotropy $[N=5]$, trace $[N=1]$, mean diffusivity $[N=1]$, radial diffusivity $[N=1])$ with DTI, WM volume $(N=6)$ and cortical thickness $(N=2)$ contained insufficient experiments for label-based review or ALE meta-analysis. There was one VBM experiment of increases in GM volume of which peak-voxel coordinates were reported, precluding ALE meta-analysis. Consequently, we performed: (i) a label-based review of studies examining GM volume; and (ii) an ALE meta-analysis of VBM studies examining reductions in GM volume.

The first author assessed suitability of studies for inclusion and used a standardized form to collect information from each study such as design, country, sample size, psychiatric morbidity and definition of violence. Any uncertainties were resolved by discussion with the other 
authors. A research assistant checked data extraction accuracy of 10 randomly selected studies. Correspondence was more than $99 \%$.

To facilitate the exploration and interpretation of results, we divided the brain into the following regions of interests (ROIs): frontal lobe; prefrontal cortex; dorsolateral prefrontal cortex (dIPFC); ventrolateral prefrontal cortex; medial prefrontal cortex; orbitofrontal cortex (OFC); anterior cingulate cortex (aCC); posterior frontal cortex; temporal lobe, lateral temporal lobe; medial temporal lobe; amygdala, hippocampus; polar temporal lobe; parietal lobe; postcentral gyrus; superior parietal lobule; inferior parietal lobule; occipital lobe; lateral occipital lobe; medial occipital lobe; cingulate cortex; posterior cingulate cortex; fusiform gyrus; temporal fusiform gyrus; occipital fusiform gyrus; striatum; and other subcortical structures (e.g. hypothalamus, cerebellum). Additional information on data extraction can be found in the supplement.

Data analysis

Label-based review

Weighting by sample size, we calculated the percentages of negative, positive and nonsignificant results reported for each brain region. Statistical significance was determined with: (i) an $\alpha$ of 0.05 (two-tailed) for results of ROI analyses; and (ii) the thresholding criteria applied by the study authors for results of VBM analyses. We rejected the null hypothesis if more than $50 \%$ of the results were all either negative or positive.

\section{ALE meta-analysis}

ALE meta-analysis was carried out in GingerALE 2.3.6 (brainmap.org/ale). We used the nonadditive algorithm ${ }^{11}$ to minimize within-experiment effects. Inference was made at cluster- 
level ( $p<.05$, with 1,000 permutations) with an uncorrected voxel-wise $p$-value of 0.005 . Cluster-level inference has been shown to provide a better balance between sensitivity and specificity compared with other methods to correct for multiple comparisons currently available in GingerALE ${ }^{12}$. The $\alpha$ levels are in line with those used in previous ALE meta-analyses ${ }^{13,14}$.

\section{Subgroup analyses}

It has been theorized that the neurobiological correlates of violent behavior differ between reactive $v s$ proactive ${ }^{15}$ and adolescence-limited vs life course-persistent ${ }^{16}$ subtypes. While we planned subgroup analyses of these subtypes for both the label-based review and ALE metaanalysis, only sufficient experiments were available to add a subgroup analysis of reactive violence to the label-based review.

Sensitivity analyses

To determine the robustness of the findings, we repeated both the label-based review and ALE meta-analysis after separately excluding experiments with: (i) reactive violence as outcome; and (ii) samples that consisted of subjects diagnosed with psychopathy ${ }^{17}$.

\section{Results}

Figure S1 shows a flow diagram of the search process. 
Chapter 6

\section{Label-based review}

There were 35 studies that met inclusion criteria for the label-based review. These studies contained a total of 1,288 participants with a mean age of 33 years (range $=20-48$ years). Most participants were male $(n=1,066,83 \%)$ and nearly half $(n=575,45 \%)$ were diagnosed with one or more of the following (classes of) psychiatric disorders: axis I disorder $(n=390$, $30 \%)$; personality disorder $(n=276,21 \%)$; schizophrenia or schizoaffective disorder $(n=244$, $19 \%) ; \operatorname{APD}(n=132,10 \%) ;$ borderline personality disorder (BPD) ( $n=115,9 \%)$; alcohol abuse or dependence ( $n=105,8 \%)$; psychopathy $(n=68,5 \%)$; intermittent explosive disorder (IED) ( $n=29,2 \%)$; and dissocial personality disorder (DPD) $(n=26,2 \%)$. For additional characteristics of the studies included in the label-based review, see Table S1.

Figure S2 shows the percentages of negative, positive and nonsignificant results reported for all brain regions $(k=86)$. Figure 1 contains the same information for the ROls $(k=28)$. The total number of results available for any one region ranged from 9 to 25 . For almost all regions, most results were nonsignificant. The parietal lobe was the only region for which more than half of the results were negative ( 7 out of $11,57 \%$ ), indicating that violence was significantly associated with reduced GM volume. For the remaining regions, negative results accounted for $46 \%$ or less of the total number of results. Percentages of positive results, reflecting a significant association between violent behavior and increased GM volume, varied between $0 \%$ and $23 \%$.

\section{Reactive violence}

Seven studies were included in the subgroup analyses of reactive violence (Table S1). These studies contained a total of 389 predominantly male $(n=362,93 \%)$ participants, who had a mean age of 26 years (range $=20-40$ years). Approximately $13 \%(n=53)$ of the participants were diagnosed with either IED ( $n=29,7 \%)$ or alcohol dependence ( $n=24,6 \%)$. The 
Figure 1. Percentages of study results indicating whether interpersonal violence was associated with a reduction, increase or no difference in grey matter volume in regions of interest.

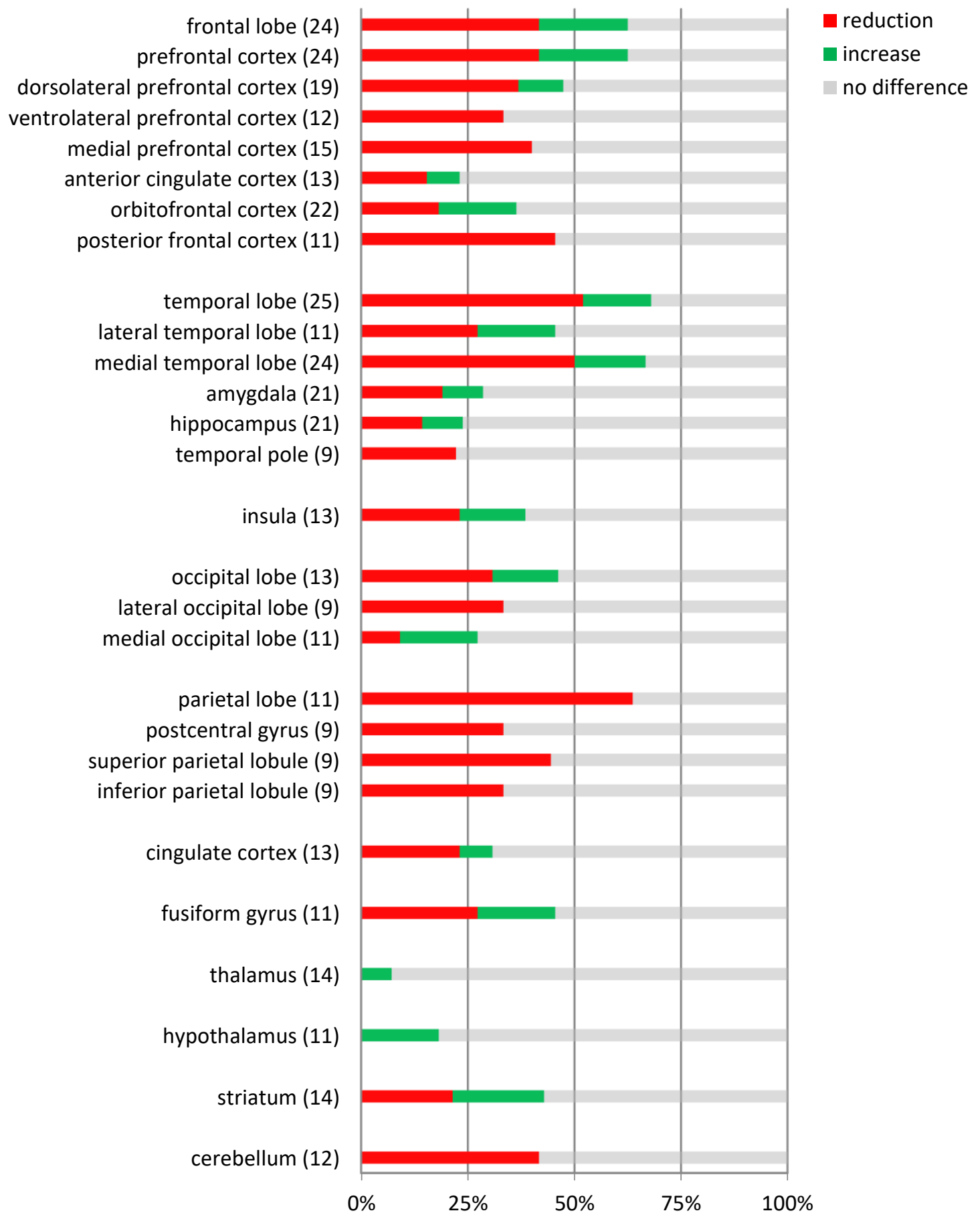

Note. For each region, the number of independent samples for which results were available is provided between parentheses. 
Chapter 6

percentages of negative and positive results for all brain regions $(k=5)$ did not exceed $37 \%$ and $18 \%$, respectively (Figure S3).

Sensitivity analyses

We observed no material differences in overall findings when repeating the label-based review after excluding experiments with reactive violence as outcome (Figure S4) or those with samples that consisted of subjects diagnosed with psychopathy (Figure S5).

\section{ALE meta-analysis}

Six VBM studies that examined reductions in regional GM volume met inclusion criteria for the ALE meta-analysis (Table S2). These studies reported 144 foci for 7 group comparisons of 13 independent samples, totaling 278 participants. The mean age was 34 years (range $=26-$ 41 years), and less than a third ( $n=90$ ) of the participants were diagnosed with one or more of the following psychiatric disorders: $\operatorname{ADP}(n=55,20 \%)$; psychopathy $(n=51,18 \%)$; $\operatorname{PPD}(n=$ $13,<1 \%) ;$ alcohol abuse or dependence $(n=26,1 \%)$; and DPD $(n=26,1 \%)$. The ALE metaanalysis showed no significant clusters of reduced GM volume in violent subjects compared with nonviolent subjects.

Sensitivity analyses

No significant clusters of reduced GM volume in comparisons between violent and nonviolent subjects were found in either sensitivity analysis. 


\section{Discussion}

To our knowledge, this is the most comprehensive review to date of neuroimaging studies investigating the association between regional GM volume and interpersonal violence. Thirtyfive studies with a total of 1,288 participants were included in a label-based review of 86 brain regions. In subgroup analyses of reactive violence, 7 studies with a total of 389 participants and 5 brain regions were included. We also performed an ALE meta-analysis of 6 VBM studies with a total of 278 participants. Neither the label-based review nor the ALE meta-analysis showed consistent associations between regional GM volume and interpersonal violence.

Our findings suggest that, in the absence of gross pathology, GM volume of discrete brain regions is not a reliable neuroimaging marker of violence. This also applies to regions implicated by current theories, including the OFC ${ }^{18}$, dIPFC ${ }^{19}, \mathrm{aCC}^{20}$, amygdala ${ }^{21}$ and hippocampus ${ }^{22}$. While most results for the parietal lobe in the label-based review indicated statistically significant reductions in GM volume, interpretation was hampered by the anatomo-functional heterogeneity of this region and the internal inconsistency of the results.

We propose phenomenological and etiological heterogeneity as the primary explanation for our findings. The construct of interpersonal violence encompasses a wide range of behaviors that arise from multifarious interactions of environment with emotional and cognitive processes (e.g. fear conditioning, impulse control, moral reasoning) mediated by different, interconnected brain regions ${ }^{23}$. It seems unlikely that all these behaviors are captured by a single neuroimaging marker. Relatedly, the failure in most studies to distinguish between subtypes (e.g. reactive vs proactive, adolescence-limited vs life course-persistent) and control for situational aspects (e.g. acute intoxication, peer group pressure) of violence may have attenuated or even obscured associations. Although the findings from the subgroup analysis suggested that reactive violence is no more consistent as outcome than generic violence, the small numbers of results and brain regions included warrant cautious interpretation. Finally, all studies relied on retrospective measurement or had potentially long time lags between scan and violent behavior. This, too, may have diminished the ability to detect associations. 
A number of important limitations to this review should be discussed. First, power is not aggregated across experiments in label-based review or ALE meta-analysis. This is compounded by the small samples often used in experiments. As a consequence, some true effects may have been missed. We decided against label-based meta-analysis of effect sizes for the following reasons: (i) as VBM studies only report effect sizes for significant results, combining VBM and ROI studies in the same meta-analysis could lead to biased estimation of the mean effect size; (ii) preliminary examination demonstrated high levels of statistical heterogeneity; and (iii) we considered the numbers of available effect sizes combined with the small sample sizes used in most ROI experiments insufficient to conduct subgroup or meta-regression analyses. Second, inclusion of subjects who were diagnosed with psychiatric disorders (e.g. schizophrenia ${ }^{24}, \mathrm{BPD}^{25}$ ) and, in some instances, treated with medication (e.g. antipsychotics ${ }^{26}$ ) may have contributed to inconsistencies in results reported for some brain regions. Finally, we conducted label-based reviews and ALE meta-analyses if at least 5 experiments were available for the same brain region and contrast of interest, respectively. While deemed necessary to improve the validity of the findings, this approach excluded potentially relevant structural parameters (e.g. fractional anisotropy, cortical thickness) and tissue classes (e.g. WM, cerebrospinal fluid).

Several implications for future research arise from this review. First, phenomenological and etiological heterogeneity of violent behavior could be reduced by distinguishing between subtypes, control for situational aspects and consideration of the emotional and cognitive processes that lie on intermediate pathways. Second, possible abnormalities in violent individuals are most rigorously investigated with whole-brain analyses of multiple combinations of structural parameter and tissue class. Finally, prospective designs with short intervals between waves (weeks or months rather than years) may improve the reliability and validity of results. 


\section{References}

1. Blair RJR. Neuroimaging of psychopathy and antisocial behavior: a targeted review. Curr Psychiatry Rep 2010; 12: 76-82.

2. Yang $Y$, Raine A. Prefrontal structural and functional brain imaging findings in antisocial, violent, and psychopathic individuals: a meta-analysis. Psychiatry Res Neuroimaging 2009; 174: 81-88.

3. Brower $\mathrm{MC}$, Price BH. Neuropsychiatry of frontal lobe dysfunction in violent and criminal behaviour: a critical review. J Neurol Neurosurg Psychiatry 2001; 71: 720-726.

4. Radua J, Mataix-Cols D. Meta-analytic methods for neuroimaging data explained. Biol Mood Anxiety Disord 2012; 2: 6.

5. Liberati A, Altman DG, Tetzlaff J, et al. The PRISMA statement for reporting systematic reviews and meta-analyses of studies that evaluate health care interventions: explanation and elaboration. PLoS Med 2009; 6: b2700.

6. Bufkin JL, Lutrell VR. Neuroimaging studies of aggressive and violent behavior: current findings and implications for criminology and criminal justice. Trauma Violence Abuse 2005; 6: 176-191.

7. Dolan MC. What imaging tells us about violence in anti-social men. Crim Behav Ment Health 2010; 20: 199-214.

8. Fabian JM. Neuropsychological and neurological correlates in violent and homicidal offenders: a legal and neuroscience perspective. Aggress Violent Behav 2010; 15: 209223.

9. Hoptman MJ, Antonius D. Neuroimaging correlates of aggression in schizophrenia: an update. Curr Opin Psychiatry 2011; 24: 100-106.

10. Patrick CJ. Psychophysiological correlates of aggression and violence: an integrative review. Philos Trans R Soc Lond B Biol Sci 2008; 363: 2543-2555.

11. Turkeltaub PE, Eickhoff SB, Laird AR, et al. Minimizing within-experiment and withingroup effects in activation likelihood estimation meta-analyses. Hum Brain Mapp 2012; 33: 1-13. 
12. Eickhoff SB, Bzdok D, Laird AR, et al. Activation Likelihood Estimation meta-analysis revisited. Neuroimage 2012; 59: 2349-2361.

13. Barron DS, Fox PM, Laird AR, et al. Thalamic medial dorsal nucleus atrophy in medial temporal lobe epilepsy: a VBM meta-analysis. Neuroimage Clin 2012; 16: 25-32.

14. Fusar-Poli $P$, Howes $O$, Bechdolf $A$, et al. Mapping vulnerability to bipolar disorder: a systematic review and meta-analysis of neuroimaging studies. J Psychiatry Neurosci 2012; 37: 170-184.

15. Rosell DR, Siever L. The neurobiology of aggression and violence. CNS Spectr 2015; 20: 254-279.

16. Moffitt TE, Caspi A. Childhood predictors differentiate life-course persistent and adolescence-limited antisocial pathways among males and females. Dev Psychopathol 2001; 13: 355-375.

17. Anderson NE, Kiehl KA. Psychopathy \& aggression: when paralimbic dysfunction leads to violence. Curr Top Behav Neurosci 2014; 17: 369-393.

18. Davidson RJ, Putnam KM, Larson CL. Dysfunction in the neural circuitry of emotion regulation - a possible prelude to violence. Science 2000; 289: 591-594.

19. Davidson RJ, Jackson DC, Kalin NH. Emotion, plasticity, context, and regulation: perspectives from affective neuroscience. Psychol Bull 2000; 126: 890-909.

20. Siever LJ. Neurobiology of aggression and violence. Am J Psychiatry 2008; 165: 429-442.

21. DeLisi M, Umphress ZR, Vaughn MG. The criminology of the amygdala. Crim Justice Behav 2009; 36: 1241-1252.

22. Yang $Y$, Glenn AL, Raine A. Brain abnormalities in antisocial individuals: implications for the law. Behav Sci Law 2008; 26: 65-83.

23. Raine A. From genes to brain to antisocial behavior. Curr Dir Psychol 2008; 17: 323-328.

24. Honea R, Crow TJ, Passingham D, et al. Regional deficits in brain volume in schizophrenia: a meta-analysis of voxel-based morphometry studies. Am J Psychiatry 2005; 162: 22332245.

25. Lis E, Greenfield B, Henry M, et al. Neuroimaging and genetics of borderline personality disorder: a review. J Psychiatry Neurosci 2007; 32: 162-173.

26. Lieberman JA, Tollefson GD, Charles C, et al. Antipsychotic drug effects on brain morphology in first-episode psychosis. Arch Gen Psychiatry 2005; 62: 361-370. 
Supplementary material 
Chapter 6

\section{Data extraction}

\section{Parcellation scheme}

We used the parcellation scheme described below to categorize results. The cerebral cortex was divided into frontal, temporal, parietal, occipital and insular lobes. The frontal lobe was subdivided in the prefrontal cortex (PFC) and posterior frontal cortex. We used the parcellation of the PFC proposed by Yang and Raine ${ }^{1}$, discerning the dorsolateral prefrontal cortex (dIPFC), ventrolateral prefrontal cortex (VIPFC), medial prefrontal cortex (mPFC), orbitofrontal cortex (OFC) and anterior cingulate cortex (aCC). The definitions of Yang and Raine ${ }^{1}$ served to delineate the dIPFC (Brodmann Areas [BAs] 8, 9, 10 and 46), vIPFC (BAs 44 and 45), mPFC (medial sections of BAs 8, 9, 10, 11 and 12) and OFC (BAs 11, 12 and 47). For the aCC (BAs 24, 25, 32 and 33), we integrated the definitions used by Hoffstaedter et al. ${ }^{2}$ and Kozslovskiy et al. ${ }^{3}$. The temporal lobe was subdivided in lateral, medial and polar aspects ${ }^{4}$. The medial temporal lobe included the amygdala and hippocampus. Results for these structures are also presented separately given their hypothesized importance for violent behavior. The parietal lobe was subdivided in the postcentral gyrus, superior parietal lobule and inferior parietal lobule. The occipital lobe was subdivided in lateral and medial aspects. Results were available for two structures that encompass parts of more than one lobe: the cingulate cortex and the fusiform gyrus. The cingulate cortex was divided in the anterior cingulate cortex $(\mathrm{aCC})$ and posterior cingulate cortex (pCC). The pCC, part of the parietal lobe, was defined as BAs 23 and $31^{2,3}$. The fusiform gyrus was divided in temporal (BAs 20, 36 and 37) and occipital (BAs 18 and 19) aspects $^{4,5}$. Results are also presented for the entire cingulate cortex and fusiform gyrus. The caudate nucleus, putamen, nucleus accumbens and subdivisions containing different parts of these regions (e.g. lentiform nucleus, ventral striatum) were grouped together as the striatum $^{6}$. Other subcortical structures, such as the hypothalamus and cerebellum, were considered separately. A result recorded for a particular brain region was also recorded for one or more brain regions of which the former is part. For example, a negative result for the parahippocampal gyrus also counted as a negative result for both the medial temporal lobe and the temporal lobe. 


\section{Derivation of labels}

We adopted the labels used by the authors in each study to identify brain regions. One label was agreed upon if different labels were used across studies for essentially the same brain region (e.g. limbic striatum and ventral striatum). Results of studies that used different labels for the ROIs described above (e.g. lateral prefrontal cortex instead of dIPFC) were classified based on the information they provided (i.e. anatomical landmarks, BAs, peak voxel coordinates). The results for two ROIs examined in a study using an ROI approach ${ }^{7}$ were not classified, because they comprised large parts of two or more ROls examined for the purpose of this review. To classify results of voxel-based morphometry (VBM) studies that used two labels for the same set of peak voxel coordinates, we used either: (i) the label that was most specific (e.g. precuneus instead of parietal lobe); or (ii), if neither label was more specific than the other, the peak voxel coordinates. Peak voxel coordinates were also used to classify results in cases where VBM studies used labels that contained parts of more than one ROI (e.g. paracentral gyrus). Alternative labels for peak voxel coordinates were obtained with the Automated Anatomical Labeling atlas ${ }^{8}$ or BA atlas implemented in MRIcron ${ }^{9}$. One result in Bertsch et al. ${ }^{10}$ was not classified, because the location of the corresponding peak voxel coordinates could not reliably be determined with either atlas.

VBM and ROI analyses

If both whole-brain VBM and ROI analyses were done in the same sample, we included the results of the former given its ability to detect highly localized tissue differences across the whole brain with minimal user bias. Volumetric changes are introduced when registering an individual's image to a standard brain template. In VBM, original volumes can be preserved by modulating the spatially normalized images. Modulation entails multiplying each voxel's probability of belonging to a specific tissue class by a scaling factor proportionate to the volumetric change introduced by the spatial normalization procedure ${ }^{11}$. If both modulated and 
unmodulated voxel-wise analyses were conducted in the same sample, the results of the former were included to optimize comparison among studies. We selected the results from the voxel-wise analysis that applied the most stringent correction for multiple comparisons. The latter two criteria also applied to VBM studies included in the Anatomical Likelihood Estimation meta-analysis. Results of ROI analyses that did not involve either segmentation or tracing of GM were only included if they involved brain regions that are wholly or largely composed of GM (e.g. prefrontal cortex, amygdala).

\section{Overlapping samples}

If more than one result was available for the same brain region in overlapping samples of unequal size, we included the result of the analysis that used the largest sample. If two or more different studies provided results for the same brain region in overlapping samples of equal size, we included the result of either: (i) the study that provided the most accurate data for the calculation of group differences ${ }^{7}$; (ii) the study conducting the analysis that adjusted for the maximum number of control variables $7,12,13$; or (iii), ceteris paribus, the most recent study ${ }^{14}$. If the same study provided more than one result for the same brain region in overlapping samples of equal size, we included either: (i) the result of the analysis that adjusted for the maximum number of control variables $7,12,15,16,17,18,19$; (ii), if the number of control variables was equal, the result of the analysis with the control variable(s) most often used in other studies ${ }^{12}$; or (iii), ceteris paribus, one result per category (e.g. negative) for which results were available $10,20,21,22$. We were unable to ascertain the presence of overlap between the samples reported on in four papers from the same research group $23,24,25,26$. These samples were treated as overlapping. Unless a study reported only bilateral results, we used the following coding scheme: (i) a nonsignificant result was recorded if the results for both hemispheres were nonsignificant; (ii) a negative or positive significant result was recorded if the results for both hemispheres were significant in a negative or positive direction, respectively; and (iii) a negative or positive significant result was recorded if the result for one hemisphere was either nonsignificant or not reported on and the result for the other hemisphere was 
significant in a negative or positive direction, respectively. An analogous approach was used to combine results for a main region (e.g. amygdala) if results were available for one or more of its subregions (e.g. ventral amygdala) in overlapping samples: (i) a nonsignificant result was recorded if the result for the main region or all results for its subregions were nonsignificant; (ii) a negative or positive significant result was recorded if for the main region or at least one of its subregions a negative or positive result was reported, respectively; and (iii) results belonging to the same category (e.g. negative) counted as one result.

\section{Multiple appropriate violent outcomes}

In instances where the same study used more than one appropriate instrument to measure violent behavior, we included the results of the analysis based on the instrument that either: (i) measured the most severe type of violent behavior ${ }^{27}$; (ii) was most often used in other studies $^{28}$; or (iii) measured violent behavior across the lifespan instead of that up to the age of $15^{29}$. Using the same instrument, one study ${ }^{16}$ conducted both correlation and betweengroup analyses. As it provided a more detailed assessment by taking into account both the frequency and severity of violent behavior, we included the result of the correlation analysis. From Pardini et al. ${ }^{30}$, we included the results of the analyses that combined self-report and criminal records for the ascertainment of violence.

\section{Psychiatric morbidity}

Psychiatric morbidity was recorded if all participants in one sample were diagnosed with the same psychiatric disorder or class of psychiatric disorders (e.g. axis I, personality disorder). Where possible, we chose appropriate psychiatric controls over healthy controls. 
Chapter 6

Analyses of variance (ANOVAs) and multivariate analyses of variance (MANOVAs)

Two-tailed t-tests with an $\alpha$ of 0.05 were used to compare a group of violent participants with a group of nonviolent participants with respect to one brain region in cases where studies provided sample sizes, means and standard deviations for both groups and: (i) used ANOVA with three or more groups, including at least one group of violent participants and at least one group of nonviolent participants, or two or more brain regions as independent variables; or (ii) used MANOVA with two or more groups, including at least one group of violent participants and at least one group of nonviolent participants, or two or more brain regions as independent variables, and two or more brain regions as dependent variables. Results of ANOVAs with two groups, one group of violent participants and one group of nonviolent participants, and one brain region as independent variable were extracted as normal.

\section{Excluded experiments}

To ensure comparability of results between studies and, given that our aim was to analyze the direct relationship between violent behavior and the structural properties of discrete brain regions, we decided to exclude: (i) interaction effects; (ii) results of analyses that statistically controlled for the effect of one or more brain regions other than the brain region of interest; (iii) ratios between different brain regions; and (iv) measures of hemispheric asymmetry. 
Figure S1. Preferred Reporting Items for Systematic Reviews and Meta-Analyses diagram showing the search strategy used to identify the studies included in the label-based review and Anatomical Likelihood Estimation (ALE) meta-analysis.
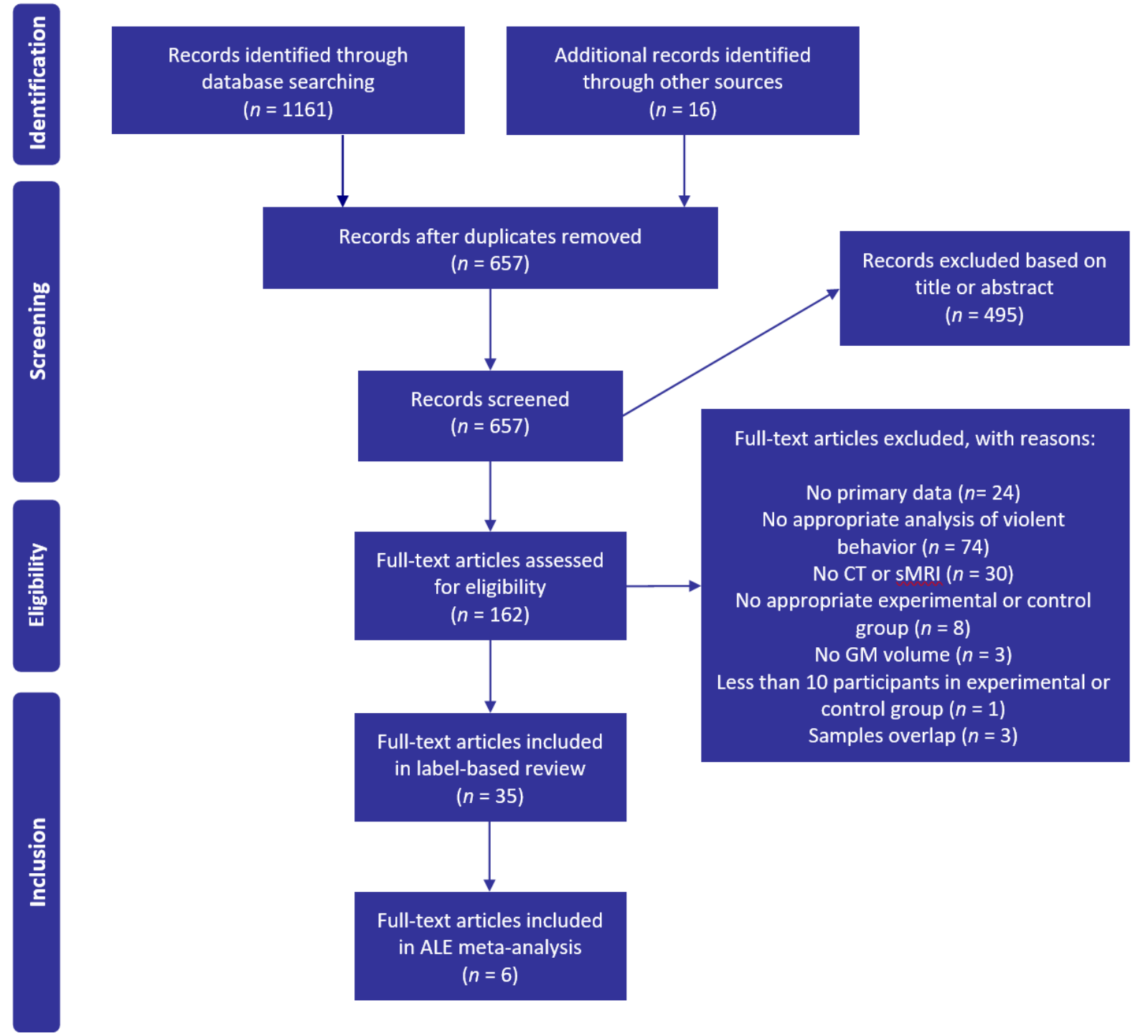

Note. $\mathrm{CT}$, computed tomography; GM, grey matter; sMRI, structural magnetic resonance imaging. Adapted from Moher et al. ${ }^{31}$. 


\section{Chapter 6}

Table S1. Characteristics of studies included in the label-based review $(N=35)$.

\begin{tabular}{|c|c|c|c|c|c|c|c|c|c|c|c|}
\hline \multirow[b]{2}{*}{ Reference } & \multirow[b]{2}{*}{ Country } & \multicolumn{5}{|c|}{ Experimental group } & \multicolumn{3}{|c|}{ Control group } & \multirow{2}{*}{$\begin{array}{l}\text { Post- } \\
\text { processing } \\
\text { technique }\end{array}$} & \multirow[b]{2}{*}{ Definition of violence } \\
\hline & & $N$ (male $n)$ & $\mathrm{Age}^{\mathrm{a}}$ & $\begin{array}{l}\text { Method of } \\
\text { recruitment }\end{array}$ & $\begin{array}{l}\text { Psychiatric } \\
\text { morbidity }\end{array}$ & $\begin{array}{l}\text { Diagnostic } \\
\text { criteria }\end{array}$ & $N($ male $n)$ & $\mathrm{Age}^{\mathrm{a}}$ & $\begin{array}{l}\text { Type of } \\
\text { control group }\end{array}$ & & \\
\hline Barkataki et al. ${ }^{7}[1]$ & U.K. & $13(13)$ & 34.46 & Institutional & $\mathrm{Sz}$ & DSM-IV & $15(15)$ & 34.47 & Psychiatric & ROI & $\begin{array}{l}\text { A minimum score of } 4 \text { on the GRS, indicating a } \\
\text { (near) fatal act of violence against another person }\end{array}$ \\
\hline Barkataki et al.? [2] & U.K. & $13(13)$ & 31.62 & Institutional & APD & DSM-IV & $15(15)$ & 32.13 & Healthy & ROI & $\begin{array}{l}\text { A minimum score of } 4 \text { on the GRS, indicating a } \\
\text { (near) fatal act of violence against another person }\end{array}$ \\
\hline Bertsch et al. ${ }^{10}[1]$ & Germany & $12(12)$ & 27.30 & Institutional & $\begin{array}{l}\text { APD, } \\
\text { Psych }\end{array}$ & $\begin{array}{l}\text { DSM-IV, } \\
\text { PCL-R }\end{array}$ & $14(14)$ & 26.10 & Healthy & VBM & $\begin{array}{l}\text { Conviction for crime that causes severe injury, } \\
\text { such as murder, manslaughter, robbery or rape }\end{array}$ \\
\hline Bertsch et al. ${ }^{10}[2]$ & Germany & $13(13)$ & 28.90 & Institutional & $\begin{array}{l}\text { APD, } \\
\text { BPD }\end{array}$ & DSM-IV & $14(14)$ & 26.10 & Healthy & VBM & $\begin{array}{l}\text { Conviction for crime that causes severe injury, } \\
\text { such as murder, manslaughter, robbery or rape }\end{array}$ \\
\hline Bobes et al.20,b & Mexico & $25(25)$ & 30.63 & Community & None & na & $29(29)$ & 28.93 & na & VBM & $\begin{array}{l}\text { A minimum score of } 9 \text { on the Reactive Aggression } \\
\text { subscale of the RPAQ }\end{array}$ \\
\hline Contreras-Rodríguez et al. ${ }^{32}$ & Spain & $22(22)$ & 39.80 & Institutional & Psych & PCL-R & $22(22)$ & 40.60 & Healthy & VBM & $\begin{array}{l}\text { Severe criminal offense history (i.e. murder an } \\
\text { armed robbery) }\end{array}$ \\
\hline Dolan et al. ${ }^{15}$ & U.K. & $17(17)$ & ns & Institutional & $\begin{array}{l}\text { Psych, } \\
\text { PD }\end{array}$ & $\begin{array}{l}\text { DSM-III-R, } \\
\text { SHAPS }\end{array}$ & $12(12)$ & ns & Healthy & $\mathrm{ROI}$ & Conviction for a violent offense, mostly murder \\
\hline Dolan et al. ${ }^{33}$ & U.K. & $43(43)$ & ns & Mixed & None & na & na & na & na & $\mathrm{ROI}$ & Total score on the BGA \\
\hline Frankle et al. ${ }^{23, b}$ & U.S.A. & $10(5)$ & 35.00 & Community & $\begin{array}{l}\text { IED-R, } \\
\text { PD }\end{array}$ & $\begin{array}{l}\text { Coc, } \\
\text { DSM-IV }\end{array}$ & $10(5)$ & 34.00 & Healthy & ROI & Meeting diagnostic criteria for IED-R \\
\hline Gansler et al..$^{12}[1]$ & U.S.A. & $41(36)$ & 40.12 & Mixed & Axis 1 & DSM-IV & na & na & na & ROI & $\begin{array}{l}\text { Score on the subscale for verbal and physical } \\
\text { aggression of the LHA-R }\end{array}$ \\
\hline Gansler et al. ${ }^{12}[2]$ & U.S.A. & $19(18)$ & 40.94 & Community & None & na & na & na & na & ROI & $\begin{array}{l}\text { Score on the subscale for verbal and physical } \\
\text { aggression of the LHA-R }\end{array}$ \\
\hline
\end{tabular}


Table S1. (Continued)

\begin{tabular}{|c|c|c|c|c|c|c|c|c|c|c|c|}
\hline \multirow[b]{2}{*}{ Reference } & \multirow[b]{2}{*}{ Country } & \multicolumn{5}{|c|}{ Experimental group } & \multicolumn{3}{|c|}{ Control group } & \multirow{2}{*}{$\begin{array}{l}\text { Post- } \\
\text { processing } \\
\text { technique }\end{array}$} & \multirow[b]{2}{*}{ Definition of violence } \\
\hline & & $N($ male $n)$ & Age $^{a}$ & $\begin{array}{l}\text { Type of } \\
\text { recruitment }\end{array}$ & $\begin{array}{l}\text { Psychiatric } \\
\text { morbidity }\end{array}$ & $\begin{array}{l}\text { Diagnostic } \\
\text { criteria }\end{array}$ & $N($ male $n)$ & $\mathrm{Age}^{\mathrm{a}}$ & $\begin{array}{l}\text { Type of } \\
\text { control group }\end{array}$ & & \\
\hline Gansler et al. ${ }^{34}$ & U.S.A. & $36(36)$ & 39.47 & Mixed & Axis I & DSM-IV & na & na & na & $\mathrm{ROI}$ & Score on the Aggression subscale of the LHA-R \\
\hline Gilliam $^{13, b}$ & U.S.A. & 169 (169) & 20.00 & Community & None & na & na & na & na & $\mathrm{ROI}$ & $\begin{array}{l}\text { Frequency of reactive aggressive acts within } \\
\text { the past year, measured with a subset of items } \\
\text { from the SRD }\end{array}$ \\
\hline Gilliam et al. ${ }^{35}$ & U.S.A. & $169(169)$ & 20.00 & Community & None & na & na & na & na & ROI & $\begin{array}{l}\text { Frequency of reactive aggressive acts within the } \\
\text { past year, measured with a subset of items from } \\
\text { the SRD }\end{array}$ \\
\hline Gregory et al. ${ }^{21}[1]$ & U.K. & $17(17)$ & 38.90 & Institutional & $\begin{array}{l}\text { APD, } \\
\text { Psych }\end{array}$ & $\begin{array}{l}\text { DSM-IV, } \\
\text { PCL-R }\end{array}$ & $22(22)^{c}$ & 32.40 & Healthy & VBM & $\begin{array}{l}\text { History of one or more convictions for violent } \\
\text { crime (i.e. murder, attempted murder, rape and } \\
\text { grievous bodily harm) }\end{array}$ \\
\hline Gregory et al. ${ }^{21}[2]$ & U.K. & $27(27)^{d}$ & 36.10 & Institutional & APD & DSM-IV & $22(22)^{c}$ & 32.40 & Healthy & VBM & $\begin{array}{l}\text { History of one or more convictions for violent } \\
\text { crime (i.e. murder, attempted murder, rape and } \\
\text { grievous bodily harm) }\end{array}$ \\
\hline Gopal et al. ${ }^{36}$ & U.S.A. & $41(36)$ & 40.00 & Mixed & Axis I & DSM-IV & na & na & na & ROI & $\begin{array}{l}\text { Score on the subscale for verbal and physical } \\
\text { aggression of the LHA-R }\end{array}$ \\
\hline Hoptman et al. ${ }^{28}$ & U.S.A. & $49(43)$ & 41.50 & Institutional & $\begin{array}{l}\text { Sz, } \\
\text { SAD }\end{array}$ & DSM-IV & na & na & na & $\mathrm{ROI}$ & $\begin{array}{l}\text { Total weighted score on the OAS, reflecting the } \\
\text { seriousness and frequency of violent incidents } \\
\text { during the study period }\end{array}$ \\
\hline Hoptman et al. ${ }^{16}$ & U.S.A. & $49(43)$ & 41.50 & Institutional & $\begin{array}{l}\text { Sz, } \\
\text { SAD }\end{array}$ & DSM-IV & na & na & na & $\mathrm{ROI}$ & $\begin{array}{l}\text { Total weighted score on the OAS, reflecting the } \\
\text { seriousness and frequency of violent incidents } \\
\text { during the study period }\end{array}$ \\
\hline Kumari et al. ${ }^{37}$ & U.K. & $10(10)$ & 35.00 & Institutional & Sz & DSM-IV & $14(14)$ & 33.80 & Psychiatric & ROI & $\begin{array}{l}\text { A score of } 5 \text { or above on the GRS, indicating at } \\
\text { least one (near) fatal act of violence against the } \\
\text { victim }\end{array}$ \\
\hline
\end{tabular}




\section{Chapter 6}

Table S1. (Continued)

\begin{tabular}{|c|c|c|c|c|c|c|c|c|c|c|c|}
\hline \multirow[b]{2}{*}{ Reference } & \multirow[b]{2}{*}{ Country } & \multicolumn{5}{|c|}{ Experimental group } & \multicolumn{3}{|c|}{ Control group } & \multirow{2}{*}{$\begin{array}{l}\text { Post- } \\
\text { processing } \\
\text { technique }\end{array}$} & \multirow[b]{2}{*}{ Definition of violence } \\
\hline & & $N($ male $n)$ & $\mathrm{Age}^{\mathrm{a}}$ & $\begin{array}{l}\text { Type of } \\
\text { recruitment }\end{array}$ & $\begin{array}{l}\text { Psychiatric } \\
\text { morbidity }\end{array}$ & $\begin{array}{l}\text { Diagnostic } \\
\text { criteria }\end{array}$ & $N($ male $n)$ & $\mathrm{Age}^{\mathrm{a}}$ & $\begin{array}{l}\text { Type of } \\
\text { control group }\end{array}$ & & \\
\hline Kumari et al. ${ }^{14}[1]$ & U.K. & $13(13)$ & 34.46 & Institutional & Sz & DSM-IV & $15(15)$ & 34.47 & Psychiatric & ROI & $\begin{array}{l}\text { A minimum score of } 4 \text { on the GRS, indicating a } \\
\text { (near) fatal act of violence against another person }\end{array}$ \\
\hline Kumari et al. ${ }^{14}[2]$ & U.K. & $13(13)$ & 31.61 & Institutional & APD & DSM-IV & $15(15)$ & 32.13 & Healthy & ROI & $\begin{array}{l}\text { A minimum score of } 4 \text { on the GRS, indicating a } \\
\text { (near) fatal act of violence against another person }\end{array}$ \\
\hline Kumari et al..$^{38}$ & U.K. & $57(57)$ & 33.07 & Mixed & None & na & na & na & na & ROI & Score on the GRS \\
\hline Laakso et al. ${ }^{39}$ & Finland & $19(19)$ & 30.00 & Institutional & $\begin{array}{l}\text { APD, } \\
\text { Alc-II }\end{array}$ & $\begin{array}{l}\text { Clo, } \\
\text { DSM-IV, } \\
\text { ICD-10 }\end{array}$ & $17(17)$ & 48.00 & Psychiatric $^{e}$ & ROI & $\begin{array}{l}\text { Charged with a violent offense (i.e. murder, } \\
\text { attempted murder, manslaughter, assisting } \\
\text { manslaughter, armed robbery, assault and } \\
\text { aggravated assault) }\end{array}$ \\
\hline Laakso et al. ${ }^{17}$ & Finland & $24(24)$ & 31.00 & Institutional & $\begin{array}{l}\text { APD, } \\
\text { Alc-II }\end{array}$ & $\begin{array}{l}\text { Clo, } \\
\text { DSM-IV, } \\
\text { ICD-10 }\end{array}$ & $33(33)$ & 34.00 & Healthy & ROI & $\begin{array}{l}\text { Charged with a violent offense (i.e. murder, } \\
\text { attempted murder, manslaughter, assisting } \\
\text { manslaughter, armed robbery, assault and } \\
\text { aggravated assault) }\end{array}$ \\
\hline Matthies et al. ${ }^{40}$ & Germany & $20(0)^{f}$ & 27.20 & Community & None & na & na & na & na & $\mathrm{ROI}$ & $\begin{array}{l}\text { Score on the subscale for verbal and physical } \\
\text { aggression of the LHA-R }\end{array}$ \\
\hline New et al. ${ }^{24, b}$ & U.S.A. & $26(19)$ & 33.97 & ns & $\begin{array}{l}\text { IED-R, } \\
\text { BPD }\end{array}$ & $\begin{array}{c}\text { Coc, } \\
\text { DSM-IV }\end{array}$ & $24(15)^{\mathrm{g}}$ & 32.56 & Healthy & ROI & Meeting diagnostic criteria for IED-R \\
\hline Pardini et al. ${ }^{30}[1]$ & U.S.A. & $21(21)$ & 26.00 & Community & None & na & $35(35)$ & 26.00 & na & ROI & $\begin{array}{l}\text { Reporting an act of violence on the SRD and/or } \\
\text { being charged with a violent crime within } 3 \text { years } \\
\text { after the scan }\end{array}$ \\
\hline
\end{tabular}


Table S1. (Continued)

\begin{tabular}{|c|c|c|c|c|c|c|c|c|c|c|c|}
\hline \multirow[b]{2}{*}{ Reference } & \multirow[b]{2}{*}{ Country } & \multicolumn{5}{|c|}{ Experimental group } & \multicolumn{3}{|c|}{ Control group } & \multirow{2}{*}{$\begin{array}{l}\text { Post- } \\
\text { processing } \\
\text { technique }\end{array}$} & \multirow[b]{2}{*}{ Definition of violence } \\
\hline & & $N($ male $n)$ & Age $^{a}$ & $\begin{array}{l}\text { Type of } \\
\text { recruitment }\end{array}$ & $\begin{array}{l}\text { Psychiatric } \\
\text { morbidity }\end{array}$ & $\begin{array}{l}\text { Diagnostic } \\
\text { criteria }\end{array}$ & $N($ male $n)$ & $\mathrm{Age}^{\mathrm{a}}$ & $\begin{array}{l}\text { Type of } \\
\text { control group }\end{array}$ & & \\
\hline Pardini et al. ${ }^{30, b}[\mathrm{II}]$ & U.S.A. & $56(56)$ & 26.00 & Community & None & na & na & na & na & ROI & $\begin{array}{l}\text { Score on the subscale for impulsive aggression of } \\
\text { the IAR }\end{array}$ \\
\hline Puri et al. ${ }^{41}$ & U.K. & $13(12)$ & 40.40 & Institutional & Sz & DSM-IV & $13(10)$ & 32.60 & Psychiatric & VBM & $\begin{array}{l}\text { Violent offending (i.e. homicide, attempted } \\
\text { homicide and wounding with intent to cause } \\
\text { grievous bodily harm) prior to admission, } \\
\text { considered by court-accepted expert opinion to } \\
\text { be a direct result of schizophrenia }\end{array}$ \\
\hline Rosell et al. . $^{2, b}[1]$ & U.S.A. & $14(10)$ & 36.75 & Community & $\begin{array}{l}\text { IED-IR+A2, } \\
\text { PD }\end{array}$ & $\begin{array}{l}\text { DSM-IV, } \\
\text { McC }\end{array}$ & $25(15)$ & 32.86 & Healthy & ROI & Meeting diagnostic criteria for IED-IR \\
\hline Rosell et al. ${ }^{25, b}[2]$ & U.S.A . & $15(12)$ & 36.40 & Community & $\begin{array}{l}\text { IED-IR-A2, } \\
\text { PD }\end{array}$ & $\begin{array}{l}\text { DSM-IV, } \\
\text { McC }\end{array}$ & $25(15)$ & 32.86 & Healthy & ROI & Meeting diagnostic criteria for IED-IR \\
\hline Schiffer et al. ${ }^{42}$ & Germany & $24(24)$ & 36.90 & Institutional & None & na & $27(27)$ & 36.99 & na & VBM & Conviction for a violent offense \\
\hline Schiffer et al..$^{29}[1]$ & Germany & $50(50)$ & 35.97 & Mixed & $\mathrm{Sz}$ & DSM-IV & na & na & na & ROI & $\begin{array}{l}\text { Composite score on the fighting and assault items } \\
\text { of the LHA-R }\end{array}$ \\
\hline Schiffer et al. ${ }^{29}[2]$ & Germany & $52(52)$ & 34.56 & Mixed & None & na & na & na & na & ROI & $\begin{array}{l}\text { Composite score on the fighting and assault items } \\
\text { of the LHA-R }\end{array}$ \\
\hline Spoletini et al. ${ }^{27}$ & Italy & $50(29)$ & 40.67 & Community & $\mathrm{Sz}$ & DSM-IV-TR & na & na & na & ROI & $\begin{array}{l}\text { Score on the Physical Aggression subscale of the } \\
\text { OAS-M }\end{array}$ \\
\hline Soloff et al. ${ }^{43}[1]$ & U.S.A. & $16(5)$ & 36.10 & Community & BPD & $\begin{array}{l}\text { DIB, DIB-R } \\
\text { ICD-10 }\end{array}$ & na & na & na & SVC & Total score on the BGA \\
\hline Soloff et al. ${ }^{43}[2]$ & U.S.A. & $35(5)$ & 27.40 & Community & BPD & $\begin{array}{l}\text { DIB, DIB-R } \\
\text { ICD-10 }\end{array}$ & na & na & na & SVC & Total score on the BGA \\
\hline
\end{tabular}




\section{Chapter 6}

Table S1. (Continued)

\begin{tabular}{|c|c|c|c|c|c|c|c|c|c|c|c|}
\hline \multirow[b]{2}{*}{ Reference } & \multirow[b]{2}{*}{ Country } & \multicolumn{5}{|c|}{ Experimental group } & \multicolumn{3}{|c|}{ Control group } & \multirow{2}{*}{$\begin{array}{l}\text { Post- } \\
\text { processing } \\
\text { technique }\end{array}$} & \multirow[b]{2}{*}{ Definition of violence } \\
\hline & & $N($ male $n)$ & $\mathrm{Age}^{\mathrm{a}}$ & $\begin{array}{l}\text { Type of } \\
\text { recruitment }\end{array}$ & $\begin{array}{l}\text { Psychiatric } \\
\text { morbidity }\end{array}$ & $\begin{array}{l}\text { Diagnostic } \\
\text { criteria }\end{array}$ & $N($ male $n)$ & $\mathrm{Age}^{\mathrm{a}}$ & $\begin{array}{l}\text { Type of } \\
\text { control group }\end{array}$ & & \\
\hline Tiihonen et al. ${ }^{22}$ & Finland & $26(26)$ & 32.50 & Institutional & $\begin{array}{l}\text { Alc-II, } \\
\text { APD, } \\
\text { DPD }\end{array}$ & $\begin{array}{l}\text { Clo, } \\
\text { DSM-IV, } \\
\text { ICD-10 }\end{array}$ & $25(25)$ & 34.60 & Healthy & VBM & $\begin{array}{l}\text { Charged with a violent offense (i.e. murder, } \\
\text { attempted murder, manslaughter, attempted } \\
\text { manslaughter, assisting manslaughter, assault, } \\
\text { armed robbery) }\end{array}$ \\
\hline van de Giessen et al..26,b & U.S.A. & $29(11)$ & 39.70 & ns & $\begin{array}{l}\text { IED-IR, } \\
\text { PD }\end{array}$ & $\begin{array}{l}\text { DSM-IV, } \\
\text { McC }\end{array}$ & $30(21)$ & 35.50 & Healthy & ROI & Diagnostic criteria for IED-IR \\
\hline Yang et al. ${ }^{18}[1]$ & China & $22(3)$ & 34.68 & Institutional & $\mathrm{Sz}$ & $\begin{array}{l}\text { CCMD-3, } \\
\text { DSM-IV }\end{array}$ & $19(3)$ & 33.11 & Psychiatric & $\mathrm{ROI}$ & Accused of homicide $^{h}$ \\
\hline Yang et al. ${ }^{18}[2]$ & China & $18(2)$ & 31.39 & Institutional & None & na & $32(4)$ & 32.03 & na & $\mathrm{ROI}$ & Accused of homicide $^{h}$ \\
\hline Zetzsche et al. ${ }^{44}$ & Germany & $25(0)$ & 26.10 & Institutional & BPD & $\begin{array}{l}\text { DIB-R, } \\
\text { DSM-IV }\end{array}$ & na & na & na & $\mathrm{ROI}$ & Total score on the BGA \\
\hline Zetzsche et al. ${ }^{45}$ & Germany & $25(0)$ & 26.70 & Institutional & BPD & $\begin{array}{l}\text { DIB-R, } \\
\text { DSM-IV }\end{array}$ & na & na & na & $\mathrm{ROI}$ & Total score on the BGA \\
\hline Zhang et al. ${ }^{19,6}$ & U.S.A. & $51(51)$ & 31.86 & Community & None & na & na & na & na & $\mathrm{ROI}$ & $\begin{array}{l}\text { Score on the subscale for physical aggression of } \\
\text { the CTS }\end{array}$ \\
\hline Zhang et al. ${ }^{99, b}[\mathrm{a}]$ & U.S.A. & $24(24)$ & 35.00 & Community & $A D$ & DSM-IV & $14(14)$ & 38.90 & Psychiatric & ROI & $\begin{array}{l}\text { At least two acts of physical aggression (i.e. hitting } \\
\text { punching, aggressive pushing, shoving, choking or } \\
\text { using a weapon) toward a spouse or significant } \\
\text { other in the past year. These acts were not } \\
\text { premeditated and typically associated with } \\
\text { affective symptoms (e.g. palpitations, tremors) }\end{array}$ \\
\hline
\end{tabular}

AD, alcohol dependence; Alc-II, type II alcoholism; APD, antisocial personality disorder; BGA, Brown-Goodwin Assessment for Lifetime History of Aggression ${ }^{46}$; BPD, borderline personality disorder; CCMD-3, Chinese

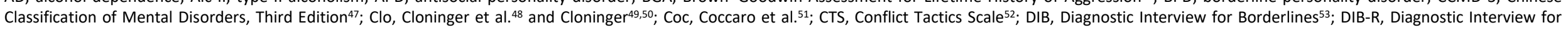
Borderlines-Revised54; DPD, dissocial personality disorder; DSM-III-R, Diagnostic and Statistical Manual of Mental Disorders, Third Edition, Revised55; DSM-IV, Diagnostic and Statistical Manual of Mental Disorders, Fourth 
Edition $^{56}$; DSM-IV-TR, Diagnostic and Statistical Manual of Mental Disorders, Fourth Edition, Text Revision ${ }^{57}$; GRS, Gunn and Robertson Scale ${ }^{58}$; IAR, Impulsive-Premeditated Aggression Scale ${ }^{59}$; ICD-10, International Classification of Diseases, 10th Revision ${ }^{60}$; IED-IR, intermittent explosive disorder-integrated research; IED-IR+A2, intermittent explosive disorder-integrated research, meeting criterion A2 (i.e. three or more acts of physical assault against other people or destruction of property over a 1-year period) for the past year; IED-IR-A2, intermittent explosive disorder-integrated research, not meeting criterion A2 (i.e. three or more acts of physical assault against other people or destruction of property over a 1-year period) for the past year; IED-R, intermittent explosive disorder-revised; LHA-R, Life History of Aggression-Revised ${ }^{61}$; McC, McCloskey et al.62; na, not applicable; ns, not specified; OAS, Overt Aggression Scale63. OAS-M, Overt Aggression Scale-Modified ${ }^{64}$. PD, personality disorder; PCL-R, Psychopathy Checklist-Revised ${ }^{65}$; Psych, psychopathy; RPAQ, Reactive and Proactive Aggression

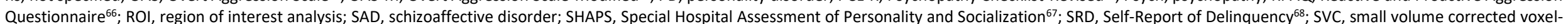
based morphometry analysis; Sz, schizophrenia; U.K., United Kingdom; U.S.A., United States of America; VBM, whole-brain voxel-based morphometry analysis.

Bracketed numbers indicate separate analyses within the same study involving different experimental groups. Bracketed Latin numerals indicate separate analyses within the same study involving the same experimental and control groups. Bracketed lower-case letters indicate separate analyses within the same study involving subsamples.

${ }^{\text {a }}$ Mean age in years

${ }^{\mathrm{b}}$ Study included in the subgroup analysis of reactive violence

${ }^{\mathrm{c}}$ Due to technical difficulties, the scans of 2 subjects were excluded

${ }^{d}$ Due to technical difficulties, the scans of 3 subjects were excluded

e Subjects were diagnosed with type I alcoholism

${ }^{f}$ Due to technical difficulties, the scans of 2 subjects were excluded and for 1 subject only the right amygdala could be traced

${ }^{\mathrm{g}}$ Due to technical difficulties, the scan of 1 subject was excluded

${ }^{h}$ While it is possible that some subjects were acquitted, it is reasonable to assume that most were convicted given the high level of correspondence between arrest and conviction rates for homicide ${ }^{69}$ 


\section{Chapter 6}

Table S2. Processing characteristics of studies included in the Anatomical Likelihood Estimation meta-analysis $(N=6)$.

\begin{tabular}{|c|c|c|c|c|c|}
\hline Reference & Software & Modulated & Coordinate system & Smoothing kernel & Thresholding criteria \\
\hline Bertsch et al. ${ }^{10}$ & SPM8 & Yes & $\mathrm{MNI}$ & $8 \mathrm{~mm}$ FWHM & $p_{\text {uncorrected }}<.005$, minimum cluster size of $67.5 \mathrm{~mm}^{3}$ \\
\hline Bobes et al. ${ }^{20}$ & SPM5 & No & $\mathrm{MNI}$ & $8 \mathrm{~mm}$ FWHM & $p_{\text {uncorrected }}<.005$ \\
\hline Contreras-Rodríguez et al. ${ }^{32}$ & SPM8 & Yes & $\mathrm{MNI}$ & $8 \mathrm{~mm}$ FWHM & $\begin{array}{l}\text { Minimum cluster size of } 1 \mathrm{~cm}^{3} \text { calculated with } 1,000 \\
\text { Monte Carlo simulations, satisfying } p_{\text {FWER }}<.05\end{array}$ \\
\hline Gregory et al. ${ }^{21}$ & SPM5 & Yes & $\mathrm{MNI}$ & $8 \mathrm{~mm}$ FWHM & $z>2.7$, cluster-corrected at $p<0.05$ using GRFT \\
\hline Schiffer et al. ${ }^{29}$ & SPM5 & Yes & $\mathrm{MNI}$ & $12 \mathrm{~mm}$ FWHM & $p_{\mathrm{FDR}}<.05$, spatial extent threshold based on GRFT \\
\hline Tiihonen et al. ${ }^{22}$ & SPM2 & ns & MNI & $12 \mathrm{~mm}$ FWHM & $p_{\mathrm{FDR}}<.05$ \\
\hline
\end{tabular}

FDR, false discovery rate; FWER, family-wise error rate; FWHM, full width at half maximum; GRFT, Gaussian random field theory; mm, millimeter; MNI, Montreal Neurological Institute; ns, not specified; SPM, statistical parametric mapping 
Figure S2. Percentages of neuroimaging experiments indicating whether interpersonal violence was associated with a reduction, increase or no difference in grey matter (GM) volume in discrete brain regions.

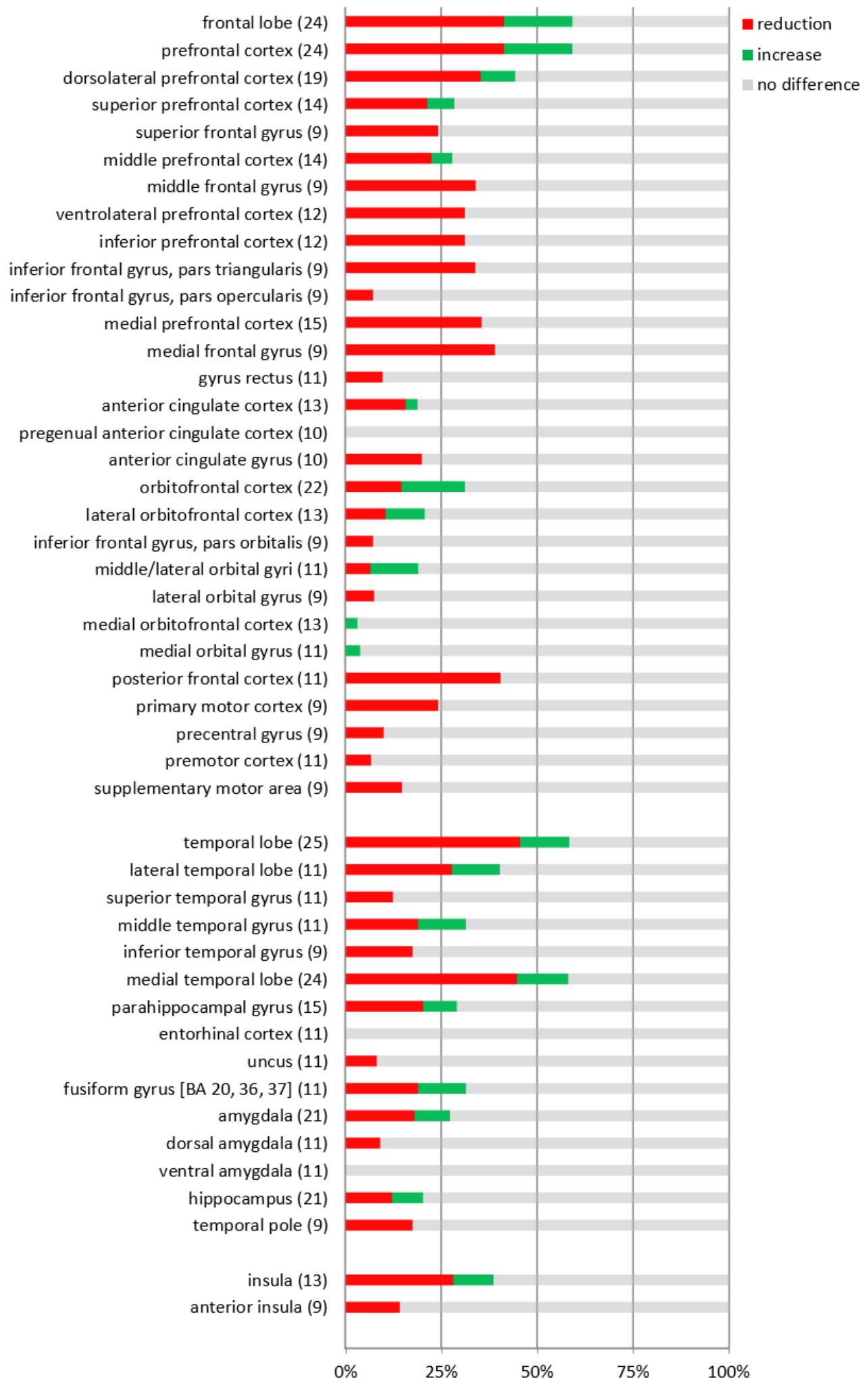




\section{Chapter 6}

Figure S2. (continued)

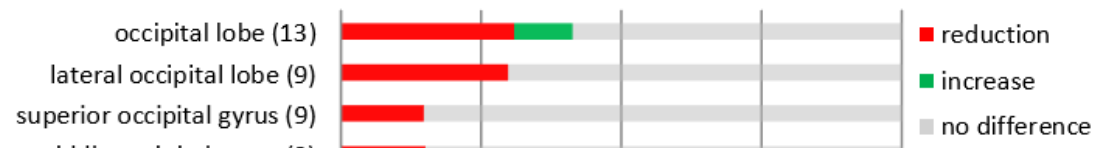

medial occipital lobe (11)

lingual gyrus (11)

fusiform gyrus [BA 18, 19] (11)

parietal lobe (11)

postcentral gyrus (9)

superior parietal lobule (9)

precuneus $(9)$

inferior parietal lobule (9)

angular gyrus (9)

supramarginal gyrus (9) posterior cingulate cortex (9)

posterior cingulate gyrus (9)

cingulate cortex (13)

anterior cingulate cortex (13)

pregenual anterior cingulate cortex (10)

anterior cingulate gyrus (10)

posterior cingulate cortex (9)

posterior cingulate gyrus (9)

fusiform gyrus (11)

fusiform gyrus [BA 18, 19] (11)

fusiform gyrus [BA 20, 37] (11)

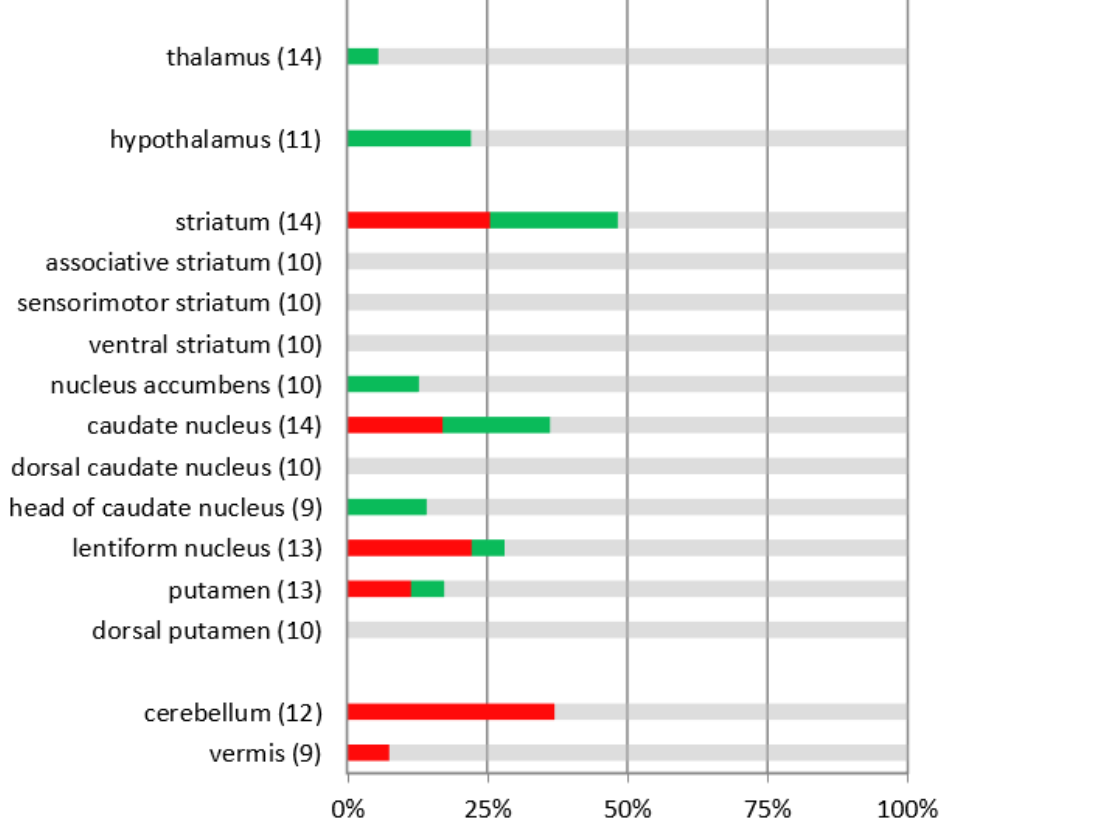

Note. BA, Brodmann Area. For each region, the total number of experiments is given between parentheses. 
Figure S3. Percentages of neuroimaging experiments indicating whether reactive violence was associated with a reduction, increase or no difference in grey matter (GM) volume in discrete brain regions.

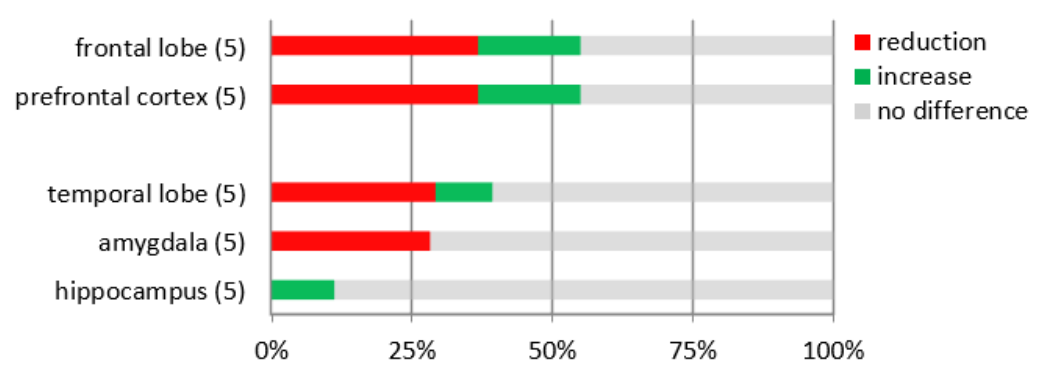

Note. For each region, the total number of experiments is given between parentheses. 


\section{Chapter 6}

Figure S4. Percentages of neuroimaging experiments indicating whether interpersonal violence was associated with a reduction, increase or no difference in grey matter (GM) volume in discrete brain regions, excluding experiments in which reactive violence was used as outcome.

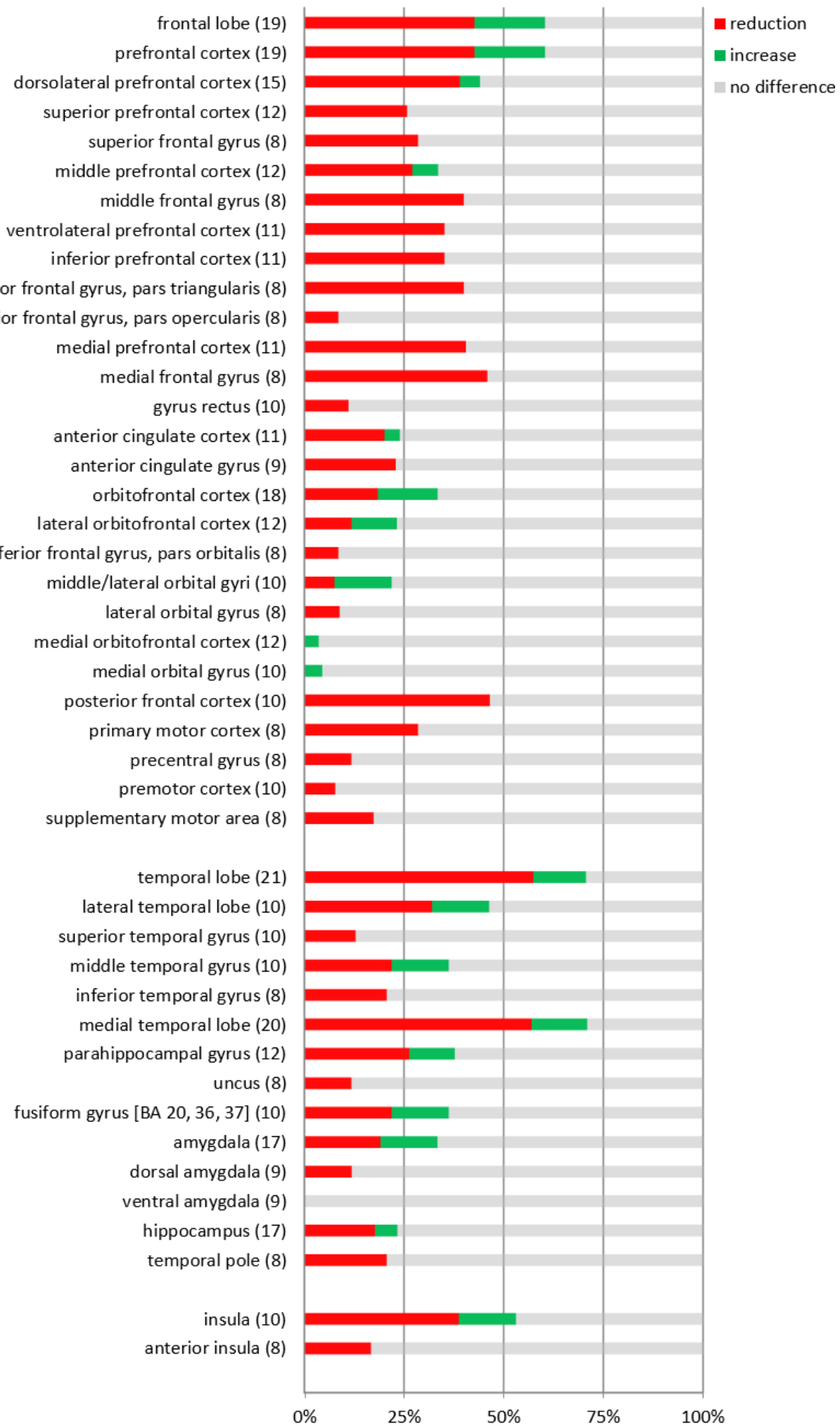


Figure S4. (continued)

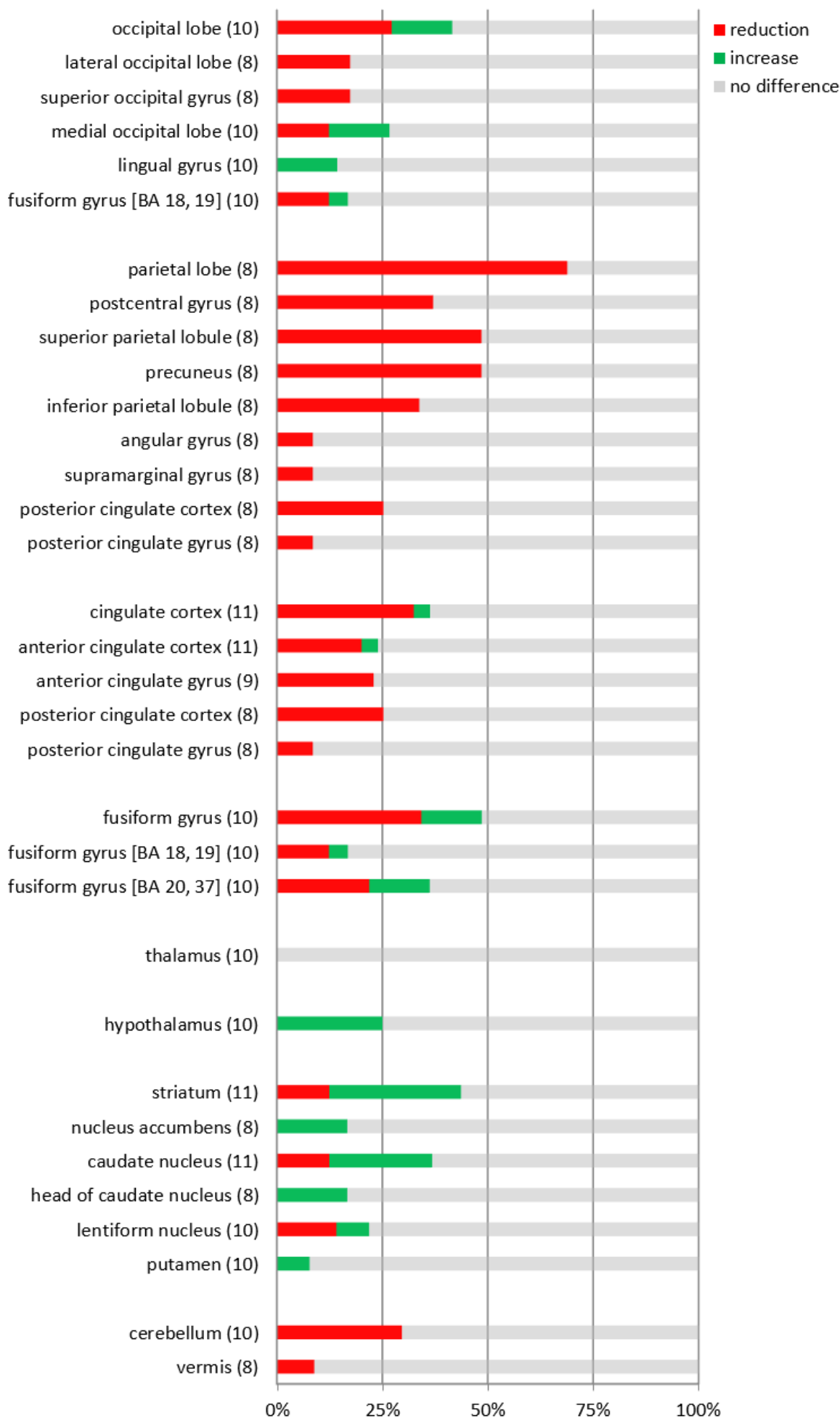

Note. For each region, the total number of experiments is given between parentheses. BA, Brodmann Area. 


\section{Chapter 6}

Figure S5. Percentages of neuroimaging experiments indicating whether interpersonal violence was associated with a reduction, increase or no difference in grey matter (GM) volume in discrete brain regions, excluding experiments with samples that consisted of subjects diagnosed with psychopathy.

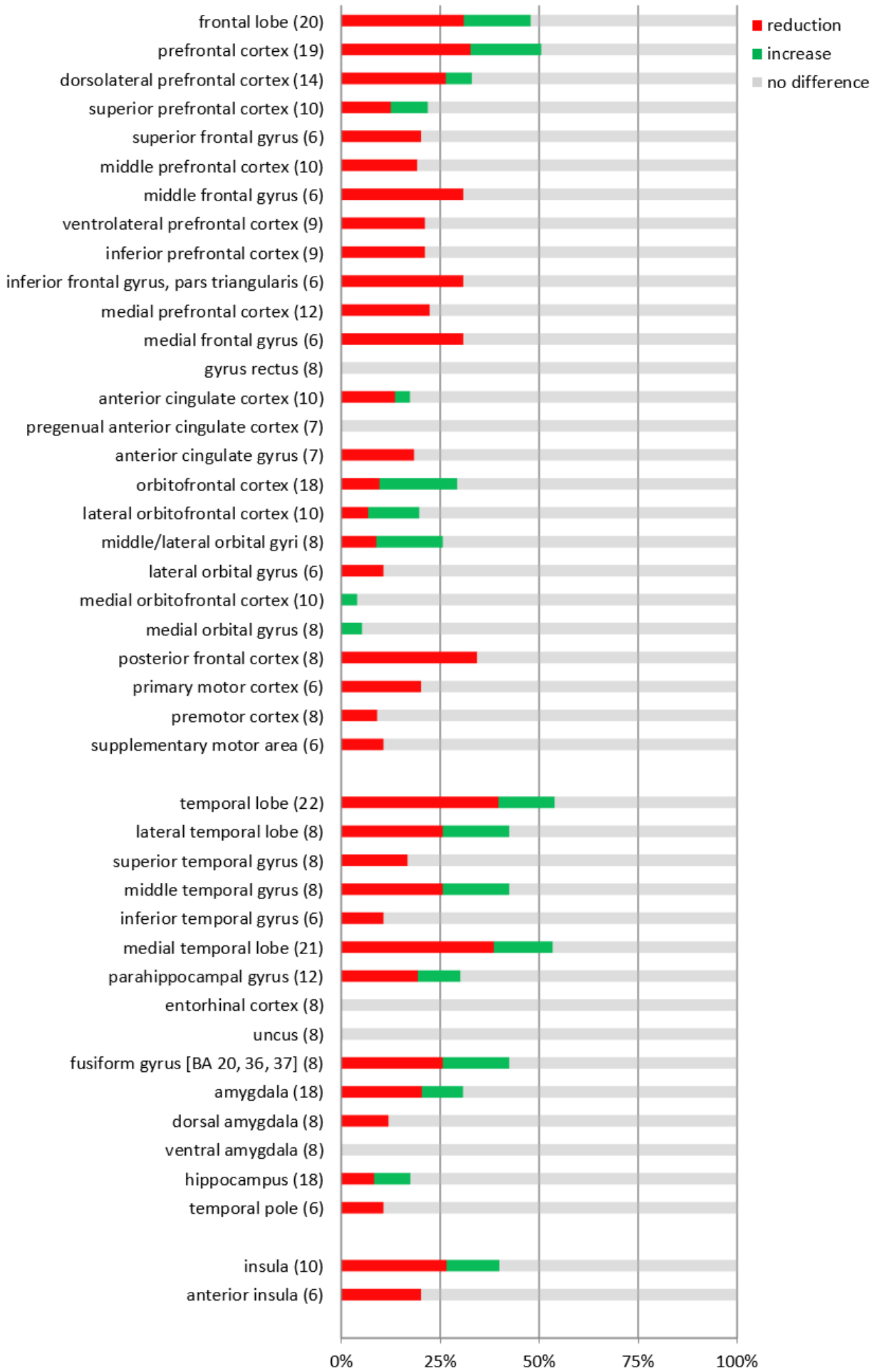


Figure S5. (continued)

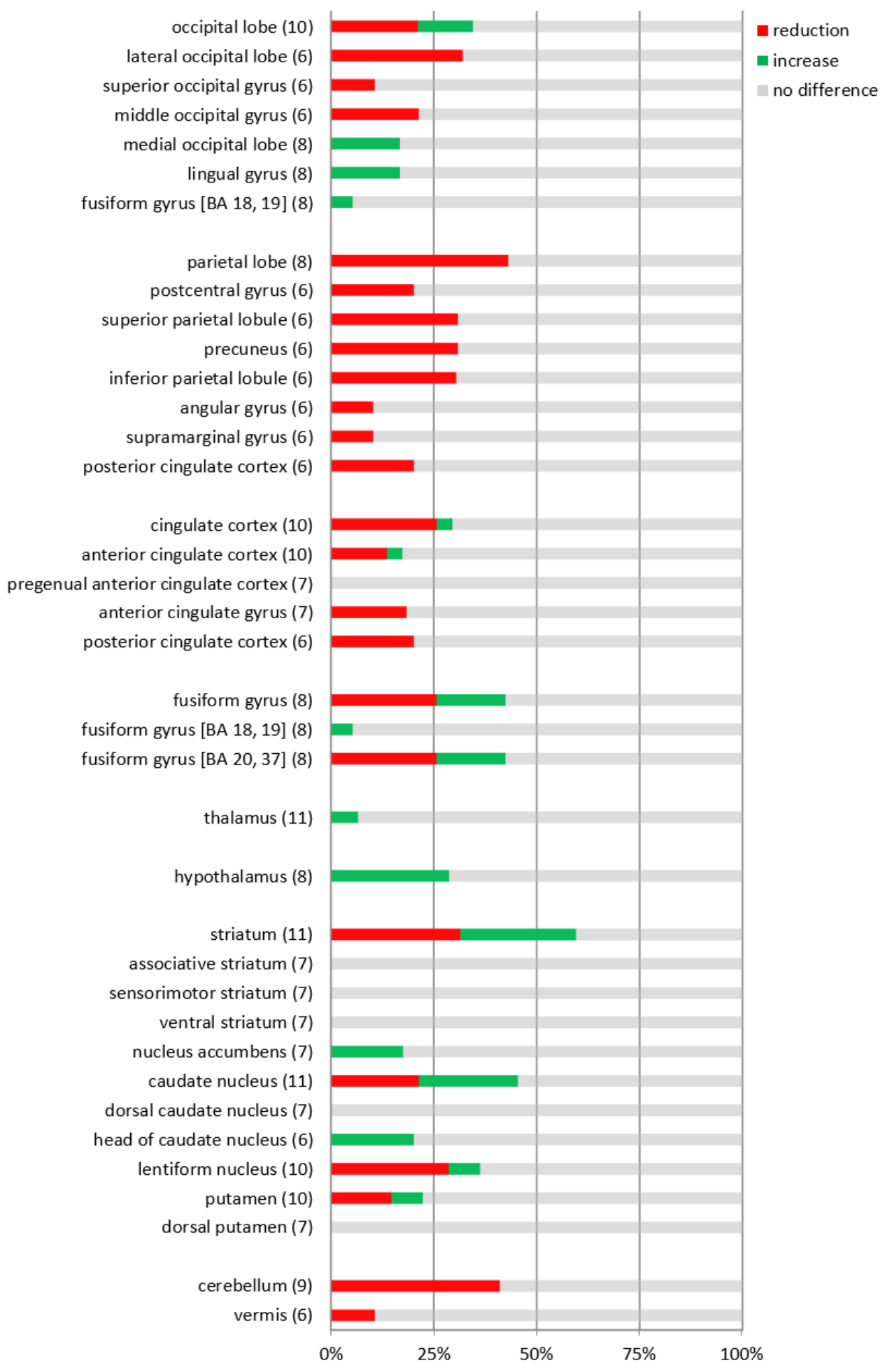

Note. For each region, the total number of experiments is given between parentheses. BA, Brodmann Area. 


\section{References}

1. Yang $Y$, Raine A. Prefrontal structural and functional brain imaging findings in antisocial, violent, and psychopathic individuals: a meta-analysis. Psychiatry Res Neuroimaging 2009; 174: 81-88.

2. Hoffstaedter F, Grefkes C, Caspers S, et al. The role of anterior midcingulate cortex in cognitive motor control: evidence from functional connectivity analyses. Hum Brain Mapp 2014; 35: 2741-2753.

3. Kozslovskiy SA, Vartanov AV, Nikonova EY, et al. The cingulate cortex and human memory processes. Psychol Russia 2012; 6: 231-243.

4. Kiernan JA. Anatomy of the temporal lobe. Epilepsy Res Treat 2012; 2: 176157.

5. Rademacher J, Galaburda AM, Kennedy DN, et al. Human cerebral cortex: localization, parcellation, and morphometry with magnetic resonance imaging. J Cogn Neurosci 1992; 4: 352-374.

6. Nolte J. The human brain: an introduction to its functional anatomy. Moby Elsevier, 2009.

7. Barkataki I, Kumari V, Das M, et al. Volumetric structural brain abnormalities in men with schizophrenia or antisocial personality disorder. Behav Brain Res 2006; 169: 239-247.

8. Tzourio-Mazoyer N, Landeau B, Papathanassiou D, et al. Automated anatomical labeling of activations in SPM using a macroscopic anatomical parcellation of the MNI MRI singlesubject brain. Neuroimage 2002; 15: 273-289.

9. Rorden $\mathrm{C}$, Karnath $\mathrm{H}-\mathrm{O}$, Bonilha L. Improving lesion-symptom mapping. J Cogn Neurosci 2007; 19: 1081-1088.

10. Bertsch K, Grothe M, Prehn K, et al. Brain volumes differ between diagnostic groups of violent criminal offenders. Eur Arch Psychiatry Clin Neurosci 2013; 263: 593-606.

11. Radua J, Canales-Rodríguez EJ, Pomarol-Clotet E, et al. Validity of modulation and optimal settings for advanced voxel-based morphometry. Neuroimage 2014: 86; 81-90.

12. Gansler DA, McLaughlin NC, Iguchi L, et al. Multivariate approach to aggression and the orbital frontal cortex in psychiatric patients. Psychiatry Res Neuroimaging 2009; 171: 145-154. 
13. Gilliam MC. Exposure to maternal depression during childhood and adolescence and problem behavior in early adulthood: a possible mediating role for brain structure (Master's thesis). University of Pittsburgh, 2014.

14. Kumari V, Gudjonsson GH, Raghuvanshi S, et al. Reduced thalamic volume in men with antisocial personality disorder or schizophrenia and a history of serious violence and childhood abuse. Eur Psychiatry 2013; 28: 225-234.

15. Dolan MC, Deakin JF, Roberts N, et al. Quantitative frontal and temporal structural MRI studies in personality-disordered offenders and control subjects. Psychiatry Res Neuroimaging 2002; 116: 133-149.

16. Hoptman MJ, Volavka J, Czobor P, et al. Aggression and quantitative MRI measures of caudate in patients with chronic schizophrenia or schizoaffective disorder. J Neuropsychiatry Clin Neurosci 2006; 18: 509-515.

17. Laakso MP, Gunning-Dixon F, Vaurio O, et al. Prefrontal volumes in habitually violent subjects with antisocial personality disorder and type 2 alcoholism. Psychiatry Res Neuroimaging 2002; 114: 95-102.

18. Yang $Y$, Raine A, Han C-B, et al. Reduced hippocampal and parahippocampal volumes in murderers with schizophrenia. Psychiatry Res Neuroimaging 2010; 182: 9-13.

19. Zhang L, Kerich $M$, Schwandt ML, et al. Smaller right amygdala in Caucasian alcoholdependent male patients with a history of intimate partner violence: a volumetric imaging study. Addict Biol 2013; 18: 537-547.

20. Bobes MA, Ostrosky F, Diaz K, et al. Linkage of functional and structural anomalies in the left amygdala of reactive-aggressive men. Soc Cogn Affect Neurosci 2013; 8: 928-936.

21. Gregory S, Ffytche D, Simmons A, et al. The antisocial brain: psychopathy matters. A structural MRI investigation of antisocial male violent offenders. Arch Gen Psychiatry 2012; 69: 962-972.

22. Tiihonen J, Rossi R, Laakso MP, et al. Brain anatomy of persistent violent offenders: more rather than less. Psychiatry Res. Neuroimaging 2008; 163: 201-212.

23. Frankle WG, Lombardo I, New AS, et al. Brain serotonin transporter distribution in subjects with impulsive aggressivity: a positron emission study with [11C]McN 5652. Am J Psychiatry 2005; 162: 915-923. 
24. New AS, Hazlett EA, Buchsbaum MS, et al. Amygdala-prefrontal disconnection in borderline personality disorder. Neuropsychopharmacology 2007; 32: 1629-1640.

25. Rosell DR, Thompson JL, Slifstein M, et al. Increased serotonin 2A receptor availability in the orbitofrontal cortex of physically aggressive personality disordered patients. Biol Psychiatry 2010; 67: 1154-1162.

26. van de Giessen E, Rosell DR, Thompson JL, et al. Serotonin transporter availability in impulsive aggressive personality disordered patients: a PET study with [11C]DASB. J Psychiatr Res 2014; 58: 147-154.

27. Spoletini I, Piras F, Fagioli S, et al. Suicidal attempts and increased right amygdala volume in schizophrenia. Schizophr Res 2011; 125: 30-40.

28. Hoptman MJ, Volavka J, Weiss EM, et al. Quantitative MRI measures of orbitofrontal cortex in patients with chronic schizophrenia or schizoaffective disorder. Psychiatry Res Neuroimaging 2005; 140: 133-145.

29. Schiffer B, Leygraf N, Müller BW, et al. Structural brain alterations associated with schizophrenia preceded by conduct disorder: a common and distinct subtype of schizophrenia? Schizophr Bull 2013; 39: 1115-1128.

30. Pardini DA, Raine A, Erickson K, et al. Lower amygdala volume in men is associated with childhood aggression, early psychopathic traits, and future violence. Biol Psychiatry 2014; 75: 73-80.

31. Moher D, Liberati A, Tetzlaff J, et al. Preferred reporting items for systematic reviews and meta-analyses: the PRISMA statement. PLoS Med 2019; 6: e1000097.

32. Contreras-Rodríguez O, Pujol J, Batalla I, et al. Functional connectivity bias in the prefrontal cortex of psychopaths. Biol Psychiatry 2014; 78: 647-655.

33. Dolan M, Deakin WJ, Roberts N, et al. Serotonergic and cognitive impairment in impulsive aggressive personality disordered offenders: are there implications for treatment? Psychol Med 2002; 32: 105-117.

34. Gansler DA, Lee AK, Emerton BC, et al. Prefrontal regional correlates of self-control in male psychiatric patients: impulsivity facets and aggression. Psychiatry Res Neuroimaging 2011; 191: 16-23. 
35. Gilliam M, Forbes EE, Gianaros PJ, et al. Maternal depression in childhood and aggression in young adulthood: evidence for mediation by offspring amygdala-hippocampal volume ratio. J Child Psychol Psychiatry 2014; 56: 1083-1091.

36. Gopal A, Clark E, Allgair A, et al. Dorsal/ventral parcellation of the amygdala: relevance to impulsivity and aggression. Psychiatry Res Neuroimaging 2013; 211: 24-30.

37. Kumari V, Barkataki I, Goswami S, et al. Dysfunctional, but not functional, impulsivity is associated with a history of seriously violent behaviour and reduced orbitofrontal and hippocampal volumes in schizophrenia. Psychiatry Res Neuroimaging 2009; 173: 39-44.

38. Kumari V, Uddin S, Premkumar $\mathrm{P}$, et al. Lower anterior cingulate volume in seriously violent men with antisocial personality disorder or schizophrenia and a history of childhood abuse. Aust N Z J Psychiatry 2014; 48: 153-161.

39. Laakso MP, Vaurio O, Savolainen L, et al. A volumetric MRI study of the hippocampus in type 1 and 2 alcoholism. Behav Brain Res 2000; 109: 177-186.

40. Matthies S, Rüsch N, Weber M, et al. Small amygdala - high aggression? The role of the amygdala in modulating aggression in healthy subjects. World J Biol Psychiatry 2012; 13: $75-81$.

41. Puri BK, Counsell SJ, Saeed N, et al. Regional grey matter volumetric changes in forensic schizophrenia patients: an MRI study comparing the brain structure of patients who have seriously and violently offended with that of patients who have not. BMC Psychiatry 2008; 8: S6.

42. Schiffer B, Müller BW, Scherbaum N, et al. Disentangling structural brain alterations associated with violent behavior from those associated with substance use disorders. Arch Gen Psychiatry 2011; 68: 1039-1049.

43. Soloff $P$, White $R$, Diwadkar VA. Impulsivity, aggression and brain structure in high and low lethality suicide attempters with borderline personality disorder. Psychiatry Res Neuroimaging 2014; 222: 131-139.

44. Zetzsche T, Preuss UW, Frodl T, et al. Hippocampal volume reduction and history of aggressive behaviour in patients with borderline personality disorder. Psychiatry Res Neuroimaging 2007; 154: 157-170. 
45. Zetzsche T, Preuss UW, Bondy B, et al. 5-HT1A receptor gene C-1019 G polymorphism and amygdala volume in borderline personality disorder. Genes Brain Behav 2008; 7: 306-313.

46. Brown GL, Goodwin FK, Ballenger JC, et al. Aggression in humans correlates with cerebrospinal fluid amine metabolites. Psychiatry Res 1979; 1: 131-139.

47. Chinese Society of Psychiatry. The Chinese Classification and Diagnostic Criteria of Mental Disorders (3rd edition). Shandong Science and Technology Press, 2001.

48. Cloninger CR, Bohman M, Sigvardsson S. Inheritance of alcohol abuse: cross-fostering analysis of adopted men. Arch Gen Psychiatry 1981; 38: 861-868.

49. Cloninger CR. Neurogenetic adaptive mechanisms in alcoholism. Science 1987; 236: 410416.

50. Cloninger CR. A systematic method for clinical description and classification of personality variants: a proposal. Arch Gen Psychiatry 1987; 44: 573-588.

51. Coccaro EF, Kavoussi RJ, Berman M, et al. Intermittent explosive disorder-revised: development, reliability, and validity of research criteria. Compr Psychiatry 1998; 39: 368-376.

52. Straus MA Measuring intrafamily conflict and violence: the conflict tactics scales. J Marriage Fam 1979; 41: 75-88.

53. Gunderson JG, Kolb JE, Austin V. The diagnostic interview for borderlines. Am J Psychiatry 1981; 138: 896-903.

54. Zanarini M, Gunderson JG, Frankenburg FR, et al. The revised diagnostic interview for borderlines: discriminating BPD from other Axis II disorders. J Pers Disord 1989; 3: 10-18.

55. American Psychiatric Association. Diagnostic and Statistical Manual of Mental Disorders (3rd edition). Author, 1987.

56. American Psychiatric Association. Diagnostic and Statistical Manual of Mental Disorders (4th edition). Author, 1994.

57. American Psychiatric Association. Diagnostic and Statistical Manual of Mental Disorders (4th edition, text revision). Author, 2000.

58. Gunn J, Robertson G. Drawing a criminal profile. Br J Criminol 1976; 16: 156-160.

59. Stanford MS, Houston RJ, Mathias CW. Characterizing aggressive behavior. Assessment 2003; 10: 183-190. 
60. World Health Organization. The ICD-10 classification of mental and behavioral disorders: clinical descriptions and diagnostic guidelines. WHO, 1992.

61. Coccaro EF, Berman ME, Kavoussi RJ. Assessment of life history of aggression: development and psychometric characteristics. Psychiatry Res 1997; 73: 147-157.

62. McCloskey MS, Berman ME, Noblett KL, et al. Intermittent explosive disorder-integrated research diagnostic criteria: convergent and discriminant validity. J Psychiatr Res 2006; 40: 231-242.

63. Yudofsky SC, Silver JM, Jackson W, et al. The overt aggression scale for the objective rating of verbal and physical aggression. Am J Psychiatry 1986; 143: 35-39.

64. Kay SR, Wolkenfeld F, Murrill LM. Profiles of aggression among psychiatric patients: nature and prevalence. J Nerv Ment Dis 1998; 176: 539-546.

65. Hare, R.D. The Hare Psychopathy Checklist-Revised. Multi-Health Systems, 2003.

66. Raine A, Dodge $K$, Loeber $R$, et al. The reactive-proactive aggression questionnaire: differential correlates of reactive and proactive aggression in adolescent boys. Aggress Behav 2006; 32: 159-171.

67. Blackburn, R. The Special Hospital Assessment of Personality and Socialization (unpublished manuscript). Ashworth Hospital, 1982.

68. Elliott DS, Huizinga D, Ageton SS. Explaining delinquency and drug use. SAGE Publishing, 1985.

69. Forst, B. Charge attrition. In D Levinson (Ed), Encyclopedia of crime and punishment. SAGE Publishing, 2002. 



\section{Chapter 7}

General discussion 


\section{Main findings}

The overall aim of this dissertation was to expand current knowledge of violent behavior in people with psychotic disorders. To this aim, five studies were undertaken.

First, I described possible causal pathways between the most replicated risk factors and violence (chapter 2). We identified more than 20 risk factors, including low educational attainment, childhood abuse, symptoms of delusions and hallucinations, substance misuse and poor illness insight, and 41 causal pathways. Risk factors often co-occur and interact with one another. Besides this complexity, causal inference is hindered by methodological aspects of research. The most important of these are retrospective measurement, reliance on inpatient samples and variation in definitions of violence.

We then reported three studies using data from the Genetic Risk and Outcome of Psychosis (GROUP) project. The sample consisted of 891 patients with psychotic disorders from 40 mental healthcare institutions in the Netherlands. Measurements took place in 2004. The first study (chapter 3) also used data for 921 individuals who had presented with a first episode of psychosis at 5 early intervention services across England between 2005 and 2009. They had partaken in the National Evaluation of the Development and Impact of Early Intervention Services (NEDEN) project. The lifetime prevalence of violent behavior in the GROUP sample was $21 \%(n=183)$. In the NEDEN sample, it was $22 \%(n=244)$. Pooling the GROUP and NEDEN data, we analyzed the effects of daily and nondaily use of pharmacologically different types of illicit substances on violent behavior. We distinguished between cannabis, stimulants, depressants and hallucinogens. It was found that, regardless of frequency, each of these substances increased violence risk. Compared with no use, the pooled odds ratios ranged from 1.7 (95\% confidence interval $[\mathrm{Cl}] 1.3-2.3)$ to $3.3(95 \% \mathrm{Cl} 2.0-5.4)$ for daily use and from 1.2 $(95 \% \mathrm{Cl} 0.9-1.7)$ to $1.6(95 \% \mathrm{Cl} 1.2-2.0)$ for nondaily use. The effects were smallest for cannabis and largest for stimulants. Mechanisms by which substance use may cause violent behavior are disinhibition ${ }^{1}$ or exacerbation of psychotic symptoms ${ }^{2}$ by intoxication, acquisitive offending to sustain an addiction ${ }^{3}$ and involvement in illegal drug markets ${ }^{4}$. 
In the second study (chapter 4), we investigated the associations between neuropsychological test performance and violent behavior for a comprehensive set of cognitive functions. We included four tests of executive functions (inhibition, cognitive flexibility, problem solving, and planning) and two tests of theory of mind (ToM). The latter were used to measure cognitive and affective aspects of ToM. Violent patients were found to perform worse than nonviolent patients on nearly all tests. Differences were significant for the tests of problem solving $(F[1,847]=5.12, p=.024)$, planning $(F[1,499]=5.32, p=.022)$ and cognitive ToM $(F[1$, $839]=9.38, p=.002$ ). People with low fluid intelligence may use violence as a maladaptive solution to provocative situations ${ }^{5}$. Planning is essential for the ability to foresee the possible consequences of one's actions. When this ability is impaired, violent behavior becomes more likely ${ }^{6}$. Impairments in ToM may lead to violence through the misinterpretation of social cues $^{7}$, underregulation of negative emotions ${ }^{8}$ and lack of empathy ${ }^{9}$. However, violent behavior explained $1 \%$ or less of the variance in performance on each test. It seems, therefore, that cognitive deficits play a negligible role in the development of violence in psychotic disorders.

The third study (chapter 5) investigated environmental (parental deviance, childhood maltreatment, neighborhood disorganization) and clinical (substance misuse, impulsivity, delusions, hallucinations, lack of illness insight) risk factors for violent behavior before (VBO) and after (VAO) first-episode psychosis. We found that patients with VBO $\left(q_{730}=2.8, p=.015\right)$ and patients with VAO $\left(q_{812}=4.7, p<.001\right)$ were more impulsive compared with nonviolent patients. In addition, patients with VAO had more often misused substances $\left(\chi^{2}{ }_{1}=6.6, p=.011\right)$, experienced more severe delusions $\left(q_{808}=2.9, p=.011\right)$ and displayed poorer insight into illness $\left(q_{813}=2.8, p=.015\right)$ than nonviolent patients. These findings suggest that both VBO and VAO are primarily illness related. Impulsivity is a clinical feature of psychotic disorders and, relevant to VBO, may appear before the first psychotic episode. Three lines of evidence support this. First, individuals with psychotic disorders have consistently been found to score higher on measures of impulsivity than unaffected controls ${ }^{11}$. Second, conduct disorder ${ }^{12}$ and attention deficit hyperactivity disorder ${ }^{13}$ - mental disorders characterized by impulsivity - are precursors of psychotic disorders. Second, common brain abnormalities in psychotic disorders, such as increased dopamine synthesis in the striatum ${ }^{14}$ and disruption of frontostriatal circuits $^{15}$, are associated with impulsive behavior in the general population ${ }^{16-19}$ and precede 
first-episode psychosis by many years ${ }^{14}$. Impulsivity predisposes a person to violence by lowering their concern for its consequences ${ }^{19}$. Delusions may motivate violent behavior, especially if they induce anger ${ }^{20}$. Substance misuse and poor insight are strong predictors of treatment nonadherence ${ }^{21}$.

Finally, we carried out a systematic review of Magnetic Resonance Imaging studies measuring the association between regional grey matter (GM) volume and violence (chapter 6). We included 35 studies, which provided information for 86 brain regions in a total of 1,288 subjects. The parietal lobe was the only region for which more than half of the studies (7 out of 11) reported significant reductions. However, the results were spread over its subregions. An Anatomical Likelihood Estimation meta-analysis of 6 voxel-based morphometry studies, containing 144 foci and totaling 278 subjects, showed no significant clusters of reduced GM volume. Based on these findings, we concluded that violent behavior is etiologically too complex to be captured by a single neuroimaging marker.

\section{Strengths and limitations}

The studies using the GROUP and NEDEN data (chapters 3-5) shared three strengths. First, subjects were recruited from different types of care settings, including psychiatric hospitals, outpatient clinics and assisted living facilities, located in representative geographical areas of each country. This increased the generalizability of the results. Second, selection bias was reduced because recruitment happened independently of violent behavior. Finally, the use of multiple data sources, such as patient interviews and medical records, made the measures of violence more sensitive. However, there were several limitations. All studies had case-control designs and corrected for few potential confounders, precluding causal inference. Furthermore, no forensic patients were recruited for GROUP or NEDEN. For that reason, the findings cannot be generalized to this population. Important study-specific limitations were the inclusion of patients who had used more than one type of substance (chapter $\mathbf{3}$ ), the uncertain 
ecological validity of neuropsychological tests (chapter 4 ) and the treatment of impulsivity as a unitary construct (chapter 5).

The review of regional GM volume and violence (chapter 6 ) had two strengths. As a systematic review, it is replicable and aims to give an exhaustive summary of the evidence. In addition, we analyzed any brain region for which enough information was available. Important limitations included the low statistical power of 'vote counting' and large number of psychiatric samples.

\section{Practical implications}

Knowledge about risk factors for violent behavior in psychotic disorders, and their underlying mechanisms, is needed to develop strategies for its prediction and prevention. The prediction, or risk assessment, of violence has mostly been done with the aid of standardized tools after several studies in the 1980 s found little evidence in support of clinical judgment alone ${ }^{10}$. These tools consist of a fixed set of items assumed to be related to violent behavior. Values are assigned to each item and then combined into an estimate of the likelihood that violence will occur within a specified time period. Risk assessment tools are now routinely used in many countries ${ }^{11}$, including the Netherlands ${ }^{12}$, to inform decisions concerning the detention,

release and treatment of psychiatric patients ${ }^{13}$. Broadly, interventions in psychiatry aim to improve a patient's well-being through medication and a combination of information, exercises and talking. The latter are usually referred to as psychological interventions. Besides therapist and patient, psychological interventions may involve small groups of other patients or family members. Sometimes, help with independent living is included ${ }^{14}$. Most psychological interventions designed to prevent violent behavior are adaptations of cognitive behavioral therapy $(\mathrm{CBT})^{15}$. However, any psychological intervention may in principle be used.

The most widely used risk assessment tools in psychiatry, such as the Historical, Clinical and Risk management-2016, Level of Service Inventory-Revised ${ }^{17}$ and Violence Risk Appraisal 
Chapter 6

Guide $^{18}$, were developed for patients with any mental disorder or offenders without mental disorders. This raises questions about their applicability in patients with psychotic disorders. Although some risk factors are shared with other populations, their relative importance in psychotic disorders may be different. Moreover, risk factors unique to psychotic disorders may be omitted. The only common psychological interventions designed for psychotic disorders are psychoeducation and assertive community treatment ${ }^{19}$. However, others can be tailored to individual needs.

It is largely unknown how well existing risk assessment tools and interventions predict and prevent, respectively, violent behavior in people with psychotic disorders. Risk assessment tools have rarely been validated for this population ${ }^{20}$. Furthermore, the evidence for their ability to predict violence across psychiatric populations is mixed ${ }^{20,21}$. Antipsychotics are the mainstay of violence prevention in psychotic disorders ${ }^{22}$. There is some evidence to support this. A meta-analysis of 5 randomized controlled trials found that fewer patients were aggressive on antipsychotics $(n=9,2 \%)$ than on placebo $(n=34,12 \%)$. The corresponding risk ratio was $0.27(95 \% \mathrm{Cl} 0.15-0.52)^{23}$. Additional evidence comes from a register-based study of all people in Sweden who were diagnosed with a psychotic disorder before 2006 and prescribed antipsychotics in the 4 years thereafter $(N=34,178)$. Compared with periods when these people were not receiving any psychotropic medication, the rate of violent crime fell by $50 \%$ (hazard ratio $0.50,95 \% \mathrm{Cl} 0.41-0.61$ ) during periods when they were receiving antipsychotics ${ }^{24}$. Very few studies have evaluated the effectiveness of psychological interventions for preventing violence in psychotic disorders. These studies have been nonrandomized, inclusive of other mental disorders or unreplicated ${ }^{25,26}$. Considering this lack of high-quality evaluations, the prevention of violent behavior in people with psychotic disorders with psychological interventions needs to rely on indirect evidence.

Several implications for clinical or legal practice arise from the studies in this dissertation. The first study (chapter 2) makes clear that clinicians should screen patients for a wide range of risk factors to help ensure that appropriate interventions are chosen for its prevention. To the same end, they should consider possible mechanisms by which relevant risk factors led to 
violent behavior. In the first four studies (chapters 3-5), the following risk factors were identified as useful targets for interventions: delusions, impulsivity, substance use, poor insight and treatment nonadherence. With the exception of treatment nonadherence ${ }^{27}$, evidencebased interventions are available for each of these risk factors. Delusions may be managed with antipsychotics ${ }^{28}$. To improve insight, CBT, psychoeducation or motivational interviewing may be used ${ }^{29}$. Interventions for substance use and impulsivity are discussed below.

The findings from the second study (chapter 3 ) suggest that risk assessment and interventions should target any substance use. This contrasts with current practice. Almost all risk assessment tools contain only a general item for substance use disorder (SUD ${ }^{20}$. Some tools exclude substances. The Violence Risk Appraisal Guide ${ }^{18}$, for example, asks about alcohol but not about illicit substances. Incorporating subthreshold use or excluded substances may thus improve the performance of tools. Gains may be modest, however, since the effect sizes for subthreshold use were small and patients often use more than one substance ${ }^{30}$. Given that effect sizes were similar across types of substances, creating one item for each probably adds little value. Interventions are typically used to treat SUD. In most Western countries, medications have been approved for the treatment of alcohol and opiate use disorders ${ }^{31}$. However, there is scant evidence for their effectiveness in people with psychotic disorders ${ }^{32}$. It is for this reason that clinical guidelines generally advise evidence-based psychological interventions for SUD in psychotic disorders instead of medication ${ }^{32,33}$. Psychological interventions with the strongest evidence of effectiveness in the general population are CBT, contingency management, psychoeducation, motivational interviewing and relapse prevention ${ }^{34}$. CBT and motivational interviewing seem most effective for treating SUD in psychotic disorders ${ }^{35}$. In addition, therapeutic communities have been found to reduce recidivism in offenders with SUD ${ }^{36}$. Therefore, these psychological interventions may also help prevent violent behavior in individuals with psychotic disorders and either SUD or subthreshold use.

The third study (chapter 4) showed that cognition has little relevance to violence in people with psychotic disorders. Although caution is warranted in extrapolating results from other populations, the same conclusion may be drawn from the fifth study (chapter 6) regarding regional GM volume. Both studies have important implications for legal practice. The use of 
neuroscientific evidence in criminal proceedings has grown sharply in Western countries ${ }^{37}$, including the Netherlands ${ }^{38}$, over the past two decades. However, the results suggest that it is inappropriate to explain violent crimes committed by defendants diagnosed with psychotic disorders directly from neuropsychological test scores or the size of brain regions. This is compounded by the strong persuasive effect of neuroscientific evidence on jurors and judges ${ }^{39}$ and arguably more problematic than its susceptibility to bias (e.g. reverse inference, tendency to see abnormalities where expected) and manipulation (e.g. choice of performance baselines, malingering) $)^{38,40}$.

The third study (chapter 4) also has clinical implications in that cognitive deficits are likely not useful for the prediction or prevention of violence in psychotic disorders. While current risk assessment tools do not contain items for cognitive functions ${ }^{20}$, the small effect sizes suggest that adding ones for fluid intelligence, planning and cognitive ToM would marginally improve their performance at best. Similarly, psychological interventions designed to improve these cognitive functions, such as $n$-back training ${ }^{41}$, CogniFit $^{42}$ and mentalization-based therapy ${ }^{43}$, are not expected to prevent violent behavior in many patients.

From the results of the fourth study (chapter 5), it follows that clinicians should establish whether patients became violent before or after their first psychotic episode when assessing them for risk and suitable interventions. None of the risk assessment tools currently available makes this distinction ${ }^{20}$. Both VBO and VAO may be prevented by improving impulse control. There is some evidence that antipsychotics, particularly clozapine and olanzapine, are useful for this ${ }^{44}$. As for psychological interventions, research in other psychiatric populations supports the use of $\mathrm{CBT}^{45}$. Additional targets for the prevention of VAO are SUD, delusions and lack of insight.

Many people will benefit if violence in psychotic disorders is more effectively prevented. First, and perhaps most obviously, less harm will come to victims. The emotional impact of caring for a loved one ${ }^{46}$ or treating a patient ${ }^{47}$ who is violent should also not be underestimated. The fact that victims are regularly family members or mental healthcare professionals makes this all the more poignant ${ }^{48}$. Second, it should reduce the stigmatization of people with psychotic 
disorders ${ }^{49}$. This is important, as stigma is an obstacle to their treatment and social functioning ${ }^{50}$. Of course, significant improvements in prevention are not achieved quickly. Nor is it acceptable to control news coverage of violence by psychotic individuals or easy to tackle their stereotyped portrayal as violent in books or movies ${ }^{51}$. Nevertheless, public awareness campaigns providing accurate information about the prevalence and causes of violence in psychotic disorders may help dispel misconceptions. Finally, it will reduce the economic costs to society at large. These costs are substantial. As mentioned in the introduction (chapter 1 ), violence costs the global economy about $€ 1.40$ trillion every year in medical care, productivity losses and criminal justice processes. Assuming a population attributable fraction of $5 \%{ }^{52}$, this gives a crude estimate of $€ 70$ billion for the same costs of violent behavior in psychotic disorders.

\section{Recommendations for research}

Much research on violent behavior in psychotic disorders has been limited by case-control designs and small samples of inpatients ${ }^{53}$. Case-control designs preclude causal inference because the temporal order of risk factors and violence is unknown. Furthermore, participants are selected based on having been violent (cases) or not (controls). This makes sampling susceptible to bias. Studies with small samples may lack the statistical power to detect true effects. Since many individuals with psychotic disorders are never admitted to a psychiatric hospital $^{54}$, inpatients are likely unrepresentative of this population.

There is also considerable variation in how violent behavior is defined and measured across studies. This variation undoubtedly contributes to the high levels of statistical heterogeneity observed in meta-analyses ${ }^{53,55-57}$. While all definitions cover physical abuse, some add emotional abuse or are limited to homicide. Violent behavior is usually measured with criminal records, clinical case notes or self-report ${ }^{57}$. These methods should be used in combination, as each is likely to produce incomplete information. Many victims do not report violent crimes because they think the police are unable to help or fear retaliation from the perpetrator ${ }^{58}$. 
The police may fail to identify a suspect or, if they do, the suspect may not be charged or found guilty in a subsequent trial ${ }^{59}$. In the Netherlands, for example, about $5 \%$ of violent crimes is estimated to result in a conviction ${ }^{60}$. This percentage may be especially low for violent crimes committed by people with psychotic disorders. As said before, victims are often family members or mental health professionals. Both groups have additional motives not to press charges. The former may want to prevent criminal action taken against their loved one, while the latter may consider violence part of their job ${ }^{61}$ or feel bound to confidentiality ${ }^{62}$. In addition, people with psychotic disorders may be hospitalized for violent behavior instead of imprisoned ${ }^{46}$. Clinical case notes only contain information that is relevant to a patient's treatment. Self-report measures, such as interviews and surveys, rely on the honesty of subjects.

Compounding these methodological issues is etiological complexity. As discussed in the second study (chapter 2), violent behavior in psychotic disorders may be caused by many interrelated factors. Furthermore, causal factors may differ depending on the goal (instrumental or impulsive), relationship between perpetrator and victim (family member or stranger) and nature (sexual or otherwise physical) of violence ${ }^{63}$.

The main reason behind the methodological limitations and variation in research is the widespread use of convenience samples and data collected for other purposes (secondary data) ${ }^{63}$. This, in turn, can be explained by the rarity of violence in psychotic disorders. Based on the GROUP (chapters 3-5) and NEDEN (chapter 3) data, it was estimated that at least $20 \%$ of all individuals with psychotic disorders are violent at some point in their lives. This estimate is slightly higher than those reported in most previous studies ${ }^{53}$ and likely reflects the use of multiple data sources. The lifetime prevalence of psychotic disorders in the general population is approximately $1 \%^{64}$. If both estimates are correct, then violent individuals with psychotic disorders make up only $0.2 \%$ of the general population.

Clearly, there is a need for longitudinal studies with large representative samples of individuals with psychotic disorders and violent behavior as the primary outcome. In longitudinal studies, variables are measured at intervals in the same sample. This makes it possible to 
measure risk factors and violence as they occur. To minimize bias, sampling should be independent of risk factors and violence. As many risk factors and confounders should be measured as statistical power permits. For some risk factors, in particular psychotic symptoms and substance use, the time between measurements should be relatively short. That is to say, weeks rather than years. The measurement of violent behavior should be based on more than one data source and, ideally, include contextual details.

The best way to implement these suggestions is with cohort studies of psychiatric patients diagnosed with psychotic disorders. For generalizability, patients should be recruited from a wide range of general and forensic mental healthcare settings. However, patient cohort studies have at least three limitations. First, and most importantly, risk factors and violence preceding recruitment are measured retrospectively. Second, results may be biased if refusal to participate or loss to follow-up depends on any of the variables of interest. Finally, when some patients are much older than others, age or cohort effects may be introduced. Age effects occur as people grow older, whereas cohort effects characterize people born at a particular point in time ${ }^{65}$.

In practice, stronger designs are only possible with secondary data. One option is to use data from birth cohort studies. As the name suggests, such studies follow a group of people born in the same year throughout their lives. This largely avoids sampling bias and retrospective measurement. However, rare outcomes may require unfeasibly large samples. Let us assume that, as calculated above, 1 out of every 500 individuals in the general population will develop a psychotic disorder and will be violent at some point in their life. To obtain a modest number of 50 such individuals, a birth cohort of 25,000 individuals would be required. Another option is to conduct register-based studies. Such studies link nationwide registers containing individual-level data on, among other things, sociodemographic characteristics, psychiatric diagnoses and treatment, and crime. Registers have the advantage of being readily available, reliable and complete for nearly all individuals. They also make it possible to randomly select large samples or even use the entire population of a country. However, few countries keep suffi- 
ciently detailed or comprehensive registers or allow their use for research. Notable exceptions are Denmark and Sweden ${ }^{66}$. Furthermore, violent behavior is only measured with criminal records.

Results of individual studies may be combined in meta-analyses to increase statistical power and precision. For clearer conclusions to be drawn from meta-analyses, however, sampling strategies and definitions of variables should be made more consistent across studies. This can be achieved by creating research consortiums ${ }^{63}$.

Longitudinal designs will help clarify which of the mechanisms described in the first study (chapter 2) underlie the relationship between psychotic disorders and violent behavior. Based on the other studies, more specific recommendations for research can be made. The second study (chapter $\mathbf{3}$ ) should be expanded on with analyses of sedatives, opiates, inhalants and PCP. These substances were combined with other substances for reasons of statistical power but have slightly different psychopharmacological properties ${ }^{67}$. In addition, patients who use only one substance should be analyzed separately to eliminate confounding by other substances. The third study (chapter 4 ) should be repeated with neuropsychological tests that have higher ecological validity than traditional ones. Ecological validity is the degree to which test scores can be extrapolated from the laboratory to the real world ${ }^{68}$. With this issue in mind, tests have recently been developed that assess cognitive functions through everyday activities such as finding symbols on a map, shopping for groceries, listening to winning lottery numbers or remembering the names of people in photograph ${ }^{69}$. Perhaps most promising are those using virtual reality. Virtual reality is an immersive and interactive environment generated by a computer ${ }^{70}$. Compared with pen-and-paper and other computer-based tests, virtual reality-based tests offer more complex stimuli to be presented, accurate and comprehensive measurement of performance, and experimental control ${ }^{69}$. Already, virtual reality-based tests are available for most executive functions ${ }^{69,71}$ and ToM skills ${ }^{72}$. The fourth study (chapter 5) should be built on by determining what dimensions of impulsivity are risk factors for VBO and VAO. This has clinical relevance, as a well-supported model proposes that impulsivity comprises four dissociable dimensions (lack of forethought, giving up easily, need for excitement, and rash actions when experiencing negative emotions) $)^{73}$. Furthermore, these dimensions are 
differently related to violent behavior in the general population ${ }^{74}$. There were not enough studies of regional GM volume and violence in people with psychotic disorders to add subgroup analyses of these to the review (chapter 6). Several such studies have been published since ${ }^{75-78}$. However, results are difficult to interpret because studies have generally looked at different brain regions. When a brain region has been reported on more than once, results tend to be inconsistent ${ }^{79}$. Possible reasons for this are low statistical power and variation in the reference period of violence. The strongest evidence comes from a study that was published last year ${ }^{78}$. This study pooled data from ten research institutions worldwide. These institutions form the Schizophrenia Working Group of the Enhancing Neuroimaging Genetics through Meta-Analysis consortium ${ }^{80}$. I helped one of its members, the University Medical Center Utrecht, with data preparation. The final sample consisted of 902 individuals with psychotic disorders and, based on theory, 34 regions in the cerebral cortex were selected for analysis. It was found that thinning in two regions, the temporal gyrus and midcingulate cortex, was associated with both hostility and positive symptoms within a few weeks of the scan, suggesting mediation by the latter. Notwithstanding the study's strengths, in particular the large sample size, inclusion of outpatients and close temporal proximity between variables, an important limitation is the use of hostility as a proxy for violence. It also remains unclear what, if any, subcortical regions are related to violent behavior. Both issues should be addressed by future studies. 


\section{References}

1. Kuhns JB, Clodfelter TA. Illicit drug-related psychopharmacological violence: the current understanding within a causal context. Aggress Violent Behav 2009; 14: 69-78.

2. Winklbaur B, Ebner N, Sachs G, et al. Substance abuse in patients with schizophrenia. Dialogues Clin Neurosci 2006; 8: 37-43.

3. McGinty EE, Choksy S, Wintemute GJ. The relationship between controlled substances and violence. Epidemiol Rev 2016; 38: 5-31.

4. Hodgins S. Violent behaviour among people with schizophrenia: a framework for investigations of causes, and effective treatment, and prevention. Philos Trans R Soc Lond B Biol Sci 2008; 363: 2505-2518.

5. Weiss EM. Neuroimaging and neurocognitive correlates of aggression and violence in schizophrenia. Scientifica 2012: 158646.

6. Meijers J, Harte JM, Meynen G, et al. Differences in executive functioning between violent and non-violent offenders. Psychol Med 2017; 47: 1784-1793.

7. Adshead G, Moore E, Humphrey M, et al. The role of mentalising in the management of violence. Adv Psychiatr Treat 2013; 19: 67-76.

8. Fonagy $\mathrm{P}$, Luyten $\mathrm{P}$. A developmental, mentalization-based approach to the understanding and treatment of borderline personality disorder. Dev Psychopathol 2019; 21: 1355-1381.

9. Hooker $\mathrm{Cl}$, Verosky SC, Germine LT, Knight RT, et al. Mentalizing about emotion and its relationship to empathy. Soc Cogn Affect Neurosci 2018; 3: 204-217.

10. AEgisdóttir S, White MJ, Spengler PM, et al. The meta-analysis of clinical judgment project: fifty-six years of accumulated research on clinical versus statistical prediction. Couns Psychol 2006; 34: 341-382.

11. Singh JP, Desmarais SL, Hurducas C, et al. International perspectives on the practical application of violence risk assessment: a global survey of 44 countries. Int J Forensic Ment Health 2014; 13: 193-206.

12. Boumans CE, Postulart D, van Os J. Risicotaxatie: meten is niet altijd weten. Tijdschr Psychiatr. 2015; 57: 535-538. 
13. Fazel S, Singh JP, Doll $H$, et al. Use of risk assessment instruments to predict violence and antisocial behaviour in 73 samples involving 24827 people: systematic review and metaanalysis. BMJ 2012; 345: e4692.

14. Turner DT, van der Gaag M, Karyotaki E, et al. Psychological interventions for psychosis: a meta-analysis of comparative outcome studies. Am J Psychiatry 2014; 171: 523-538.

15. McGuire J. A review of effective interventions for reducing aggression and violence. Philos Trans R Soc Lond B Biol Sci. 2008; 363: 2577-2597.

16. Douglas KS, Hart SD, Webster CD, et al. HCR20: assessing risk for violence (version 3). Simon Fraser University, 2013.

17. Andrews DA, Bonta J. LSI-R: the level of service inventory-revised. Multi-Health Systems, 1995.

18. Quinsey VL, Harris GT, Rice ME, et al. Violent offenders: appraising and managing risk (version 2). American Psychological Association, 2006.

19. Knabb JJ, Welsh RK, Graham-Howard ML. Treatment alternatives for mentally disordered offenders: a literature review. Psychol; 2: 122-131.

20. Singh JP, Serper M, Reinharth J, et al. Structured assessment of violence risk in schizophrenia and other psychiatric disorders: a systematic review of the validity, reliability, and item content of 10 available instruments. Schizophr Bull 2011; 37: 899912.

21. Ramesh $\mathrm{T}$, Igoumenou $\mathrm{A}$, Vazquez Montes $\mathrm{M}$, et al. Use of risk assessment instruments to predict violence in forensic psychiatric hospitals: a systematic review and metaanalysis. Eur Psychiatry 2018; 52: 47-53.

22. Citrome L, Volavka J. Preventing violence in patients with schizophrenia. Curr Treat Options Psych 2015; 2: 182-191.

23. Leucht S, Tardy M, Komossa K, et al. Antipsychotic drugs versus placebo for relapse prevention in schizophrenia: a systematic review and meta-analysis. Lancet 2012; 379: 2063-2071.

24. Fazel S, Zetterqvist J, Larsson $\mathrm{H}$, et al. Antipsychotics, mood stabilisers, and risk of violent crime. Lancet 2014; 384: 1206-1214.

25. Maclnnes D, Masino S. Psychological and psychosocial interventions offered to forensic mental health inpatients: a systematic review. BMJ Open 2019; 9: e024351. 
26. Rampling J, Furtado V, Winsper C, et al. Non-pharmacological interventions for reducing aggression and violence in serious mental illness: a systematic review and narrative synthesis. Eur Psychiatry 2016; 34: 17-28.

27. Barkhof E, Meijer CJ, de Sonneville LM, et al. Interventions to improve adherence to antipsychotic medication in patients with schizophrenia: a review of the past decade. Eur Psychiatry 2012; 27: 9-18.

28. Haddad PM, Correll CU. The acute efficacy of antipsychotics in schizophrenia: a review of recent meta-analyses. Ther Adv Psychopharmacol 2018; 8: 303-318.

29. Pijnenborg GH, van Donkersgoed RJ, David AS, et al. Changes in insight during treatment for psychotic disorders: a meta-analysis. Schizophr Res 2013; 144: 109-117.

30. Gregg L, Barrowclough C, Haddock G. Reasons for increased substance use in psychosis. Clin Psychol Rev 2007; 27: 494-510.

31. Douaihy AB, Kelly TM, Sullivan C. Medications for substance use disorders. Soc Work Public Health 2013; 28: 264-278.

32. Buchanan RW, Kreyenbuhl J, Kelly DL, et al. The 2009 schizophrenia PORT psychopharmacological treatment recommendations and summary statements. Schizophr Bull 2010; 36: 71-93.

33. National Institute for Health and Care Excellence. Coexisting severe mental illness (psychosis) and substance misuse: assessment and management in healthcare settings. NICE: Manchester, 2011.

34. Dutra L, Stathopoulou G, Basden SL, et al. A meta-analytic review of psychosocial interventions for substance use disorders. Am J Psychiatry 2008; 165: 179-187.

35. Bennett ME, Bradshaw KR, Catalano LT. Treatment of substance use disorders in schizophrenia. Am J Drug Alcohol Abuse 2017; 43: 377.

36. Weisburd D, Farrington DP, Gill C, et al. What works in crime prevention and rehabilitation: an assessment of systematic reviews. Criminol Public Policy 2017; 16: 415449.

37. Meixner JB. The use of neuroscience evidence in criminal proceedings. J Law Biosci 2016; 3: 330-335.

38. Merkelbach HL, Merkelbach SE. Neurobewijs in de rechtszaal? Eerst een protocol. Ned Tijdschr Geneeskd 2014; 158: A7020. 
39. Aono D, Yaffe G, Kober H. Neuroscientific evidence in the courtroom: a review. Cogn Res Princ Implic 2019; 4: 40.

40. Bigenwald $A$, Chambon V. Criminal responsibility and neuroscience: no revolution yet. Front Psychol 2019; 10: 1406.

41. Soveri A, Antfolk J, Karlsson L, et al. Working memory training revisited: a multi-level meta-analysis of n-back training studies. Psychon Bull Rev 2017; 24: 1077-1096.

42. Gigler KL, Blomeke K, Shatil E, et al. Preliminary evidence for the feasibility of at-home online cognitive training with older adults. Gerontechnology 2013; 12: 26-35.

43. Allen JG, Fonagy P. Handbook of Mentalization-Based Treatment. John Wiley, 2006.

44. Krakowski MI, Czobor P. Depression and impulsivity as pathways to violence: implications for antiaggressive treatment. Schizophr Bull 2014; 40: 886-894.

45. Battagliese G, Caccetta M, Luppino OI, et al. Cognitive-behavioral therapy for externalizing disorders: a meta-analysis of treatment effectiveness. Behav Res Ther 2015; 75: 60-71.

46. Volavka J. Violence in schizophrenia and bipolar disorder. Psychiatr Danub 2013; 25: 2433.

47. O'Rourke M, Wrigley C, Hammond S. Violence within mental health services: how to enhance risk management. Risk Manag Healthc Policy 2018; 11: 159-167.

48. Nordström A, Kullgren G. Victim relations and victim gender in violent crimes committed by offenders with schizophrenia. Soc Psychiatry Psychiatr Epidemiol 2003; 38: 326-330.

49. Torrey EF. Stigma and violence: isn't it time to connect the dots? Schizophr Bull 2011; 37: 892-896.

50. Kane JC, Elafros MA, Murray SM, et al. A scoping review of health-related stigma outcomes for high-burden diseases in low- and middle-income countries. BMC Med 2019; 17: 17 .

51. Owen PR. Portrayals of schizophrenia by entertainment media: a content analysis of contemporary movies. Psychiatr Serv 2012; 63: 655-659.

52. Fazel S, Smith EN, Chang Z, et al. Risk Factors for interpersonal violence: an umbrella review of meta-analyses. Br J Psychiatry 2018; 213: 609-614.

53. Witt K, Van Dorn RA, Fazel S. Risk factors for violence in psychosis: systematic review and meta-regression analysis of 110 studies. PLoS One 2013; 8: e55942. 
Chapter 6

54. Walsh E, Buchanan A, Fahy T. Violence and schizophrenia: examining the evidence. Br J Psychiatry 2002; 180: 490-495.

55. Fazel S, Gulati G, Linsell L, et al. Schizophrenia and violence: systematic review and metaanalysis. PloS Med 2009; 6: e100012.

56. Large MM, Nielssen O. Violence in first-episode psychosis: a systematic review and metaanalysis. Schizophr Res 2011; 125: 209-220.

57. Douglas KS, Guy LS, Hart SD. Psychosis as a risk factor for violence to others: a metaanalysis. Psychol Bull 2009; 135: 679-706.

58. Langton L, Berzofsky M, Krebs C, et al. Victimizations not reported to the police, 20062010. Bureau of Justice Statistics, 2012.

59. Charette $Y$, van Koppen V. A capture-recapture model to estimate the effects of extralegal disparities on crime funnel selectivity and punishment avoidance. Security J 2016; 29: $561-583$.

60. Wetenschappelijk Onderzoek- en Documentatiecentrum. Criminaliteit en rechtshandhaving 2018. WODC, 2019.

61. Anderson A, West SG. Violence against mental health professionals: when the treater becomes the victim. Innov Clin Neurosci 2011; 8: 34-39.

62. Harte JM, van Leeuwen ME, Theuws R. Agressie en geweld tegen hulpverleners in de psychiatrie: aard, omvang en strafrechtelijke reactive. Tijdschr Psychiatr 2013; 55: 325335.

63. Appelbaum PS. In search of a new paradigm for research on violence and schizophrenia. Am J Psychiatry 2019; 176: 677-679.

64. Moreno-Küstner B, Martín C, Pastor L. Prevalence of psychotic disorders and its association with methodological issues: a systematic review and meta-analyses. PLoS One 2018; 13: e0195687.

65. Glenn ND. Distinguishing age, period, and cohort effects. In JT Mortimer, MJ Shanahan (Eds), Handbook of the Life Course (465-476). Kluwer Academic Publishers, 2011.

66. Maret-Ouda J, Tao W, Wahlin K, et al. Nordic registry-based cohort studies: possibilities and pitfalls when combining Nordic registry data. Scand J Public Health 2017; 45: 14-19.

67. Hill SL, Thomas SH. Drugs of abuse. Medicine 2016; 44: 160-169. 
68. Dawson DR, Marcotte TD. Special issue on ecological validity and cognitive assessment. Neuropsychol Rehabil 2017; 27: 599-602.

69. Neguț A, Matu SA, Sava FA, et al. Virtual reality measures in neuropsychological assessment: a meta-analytic review. Clin Neuropsychol 2016; 30: 165-184.

70. Kardong-Edgren SS, Farra SL, Alinier G, et al. A call to unify definitions of virtual reality. Clin Simul Nurs 2019; 31: 28-34.

71. Parsons TD. Neuropsychological assessment using virtual environments: enhanced assessment technology for improved ecological validity. In S Brahnam, LC Jain (Eds), Advanced computational intelligence paradigms in healthcare 6: virtual reality in psychotherapy and assessment (271-289). Springer, 2011.

72. Canty AL, Neumann DL, Fleming J, et al. Evaluation of a newly developed measure of theory of mind: the virtual assessment of mentalising ability. Neuropsychol Rehabil 2017; 27: 834-870.

73. Whiteside SP, Lynam DR, Miller JD, et al. Validation of the UPPS impulsive behaviour scale: a four-factor model of impulsivity. Eur J Pers 2005; 19: 559-574.

74. Berg JM, Latzman RD, Bliwise NG, et al. Parsing the heterogeneity of impulsivity: a metaanalytic review of the behavioral implications of the UPPS for psychopathology. Psychol Assess 2015; 27: 1129-1146.

75. Del Bene VA, Foxe JJ, Ross LA, et al. Neuroanatomical abnormalities in violent individuals with and without a diagnosis of schizophrenia. PLoS ONE 2016; 11: e0168100.

76. Kuroki $\mathrm{N}$, Kashiwagi $\mathrm{H}$, Ota M, et al. Brain structure differences among male schizophrenic patients with history of serious violent acts: an MRI voxel-based morphometric study. BMC Psychiatry 2017; 17: 105.

77. Storvestre GB, Valnes LM, Jensen A, et al. A preliminary study of cortical morphology in schizophrenia patients with a history of violence. Psychiatry Res Neuroimaging 2019; 288 : 29-36.

78. Wong TJ, Radua J, Pomarol-Clotet E, et al. An overlapping pattern of cerebral cortical thinning is associated with both positive symptoms and aggression in schizophrenia via the ENIGMA consortium. Psychol Med 2019; 16: 1-12.

79. Fjellvang M, Grøning L, Haukvik UK. Imaging violence in schizophrenia: a systematic review and critical discussion of the MRI literature. Front Psychiatry 2018; 9: 333. 
Chapter 6

80. Thompson PM, Stein JL, Medland SE, et al. The ENIGMA consortium: large-scale collaborative analyses of neuroimaging and genetic data. Brain Imaging Behav 2014; 8: $153-182$. 


\section{Appendices}

Summary

Nederlandse samenvatting (Dutch summary)

Publications arising from this dissertation 


\section{Summary}

\section{Background}

Psychotic disorders are a group of mental disorders that cause disturbances in perception, thinking, emotion and behavior. Examples are schizophrenia, schizoaffective disorder and delusional disorder. Symptoms include delusions, hallucinations and disorganized thinking. Psychotic disorders are a leading cause of disability worldwide and, despite affecting less than $1 \%$ of the population, among the most expensive mental disorders.

Over the past 30 years, a robust association has been established between psychotic disorders and violent behavior. Studies have estimated that between $10 \%$ and $25 \%$ of patients act violently at some point in their lives. Risk estimates vary, with studies reporting odds of violence in patients that are up to seven times higher than in the general population. The population attributable fraction of violence in psychotic disorders has been estimated at $5 \%$.

However, there are five important areas of uncertainty. First, causal mechanisms are poorly understood. The main reason for this is that almost all studies have been retrospective in design. Second, it is unclear to what extent different types of illicit substances, as defined by their psychopharmacological properties, and substance use below diagnostic thresholds for substance use disorder (SUD) are related to violence. These questions have a theoretical rationale in that the psychopharmacological properties of substances modify violence risk in the general population and people with psychotic disorders are highly sensitive to the harmful effects of substances. The few studies of cannabis and stimulants have used surrogate outcomes, such as hostility and aggression, while none have investigated depressants or hallucinogens. Third, the role of cognition is largely unknown. Cognitive impairment is a hallmark of psychotic disorders. Furthermore, it has been linked to violent behavior in the general population and adverse outcomes in patients, including low educational attainment, SUD and re- 
hospitalization. Relevant studies have been limited by composite measures of cognitive functions or small selections thereof. Fourth, considerable debate exists as to whether risk factors are different for violent behavior that develops before (VBO) rather than after (VAO) the onset of illness. It is plausible that risk factors for VBO are the same as for violence in the general population and that risk factors for VAO are primarily illness related. To my knowledge, these hypotheses have never been tested. Finally, the structural brain correlates of violence in psychotic disorders remain unclear. Loss of grey matter (GM) in psychotic disorders is widespread but most pronounced in the frontal and temporal lobes, hippocampus and amygdala. This has been hypothesized to cause violent behavior through disturbances in emotional regulation, reward and avoidance learning, and decision making. Only a handful of studies have investigated this. More importantly, they have used surrogate outcomes, relied on small samples of inpatients and reported on mostly different brain regions. Studies in the general population or other psychiatric disorders may also be informative in this regard. There have been over 30 such studies. However, inconsistent results and variation in anatomical terminology hamper the interpretation of their results. Reviews have been limited to small numbers of selected brain regions and have included personality disorders, such as psychopathy and antisocial personality disorder, for which violence is not a diagnostic criterion.

A common limitation of previous studies, which adds to the uncertainties described above, is the use of inpatient samples. Such samples are likely selective, as many individuals with a psychotic disorder are never hospitalized. What is more, inpatients may differ from outpatients on risk factors for violence. In particular, rates of SUD and symptom severity tend to be higher in the former.

\section{Main findings}

The overall aim of this dissertation was to expand current knowledge of violent behavior in people with psychotic disorders. To this aim, five studies were undertaken. First, I described possible causal pathways between the most replicated risk factors and violence (chapter 2). I 
identified more than 20 risk factors, including low educational attainment, childhood abuse, symptoms of delusions and hallucinations, substance misuse and poor illness insight, and 41 causal pathways. Risk factors often co-occur and interact with one another.

I then reported on three studies using data from the Genetic Risk and Outcome of Psychosis (GROUP) project. The sample consisted of 891 patients with psychotic disorders, who were recruited from 40 mental healthcare institutions in the Netherlands. The first study (chapter 3) also used data for 921 individuals who had presented with a first episode of psychosis at one of five early intervention services across England. They participated in the National Evaluation of the Development and Impact of Early Intervention Services (NEDEN) project. Pooling the GROUP and NEDEN data, I analyzed the effects of daily and nondaily use on violent behavior for the following categories of substances: cannabis, stimulants, depressants and hallucinogens. It was found that, regardless of frequency, each of these substance categories increased violence risk. The effects were smallest for cannabis and largest for stimulants. Mechanisms by which substance use may cause violent behavior are disinhibition, exacerbation of psychotic symptoms, acquisitive offending to sustain an addiction, and involvement in illegal drug markets.

In the second study (chapter 4), I investigated the associations between neuropsychological test performance and violent behavior for a comprehensive set of cognitive functions. These included problem solving, planning and theory of mind (the ability to understand other people's thoughts). Violence explained less than $1 \%$ of the variance in performance on each test. It seems, therefore, that cognitive deficits play a negligible role in the development of violence in psychotic disorders.

The third study (chapter 5) tried to identify risk factors for violent behavior before (VBO) and after (VAO) first-episode psychosis. I found that patients with VBO and patients with VAO were more impulsive compared with nonviolent patients. In addition, patients with VAO had more often misused substances, experienced more severe delusions and displayed poorer insight into illness than nonviolent patients. These findings suggest that both VBO and VAO 
are primarily illness related. Impulsivity is a clinical feature of psychotic disorders and, relevant to VBO, often appears before the first psychotic episode. Impulsivity predisposes a person to violence by lowering their concern for its consequences. Delusions may motivate violent behavior, especially if they induce anger. Substance misuse and poor insight are strong predictors of treatment nonadherence.

Finally, I carried out a systematic review of Magnetic Resonance Imaging studies measuring the association between regional GM volume and violence (chapter 6). I included 35 studies, which provided information for 86 brain regions in a total of 1,288 subjects. The parietal lobe was the only region for which more than half of the studies reported significant reductions. However, the results were spread over its subregions. A meta-analysis showed no significant clusters of reduced GM volume. Based on these findings, I concluded that violent behavior is etiologically too complex to be captured by a single biomarker.

\section{Practical implications}

Several implications for clinical and legal practice arise from this dissertation. The first study (chapter 2) makes clear that clinicians should screen patients for a wide range of risk factors to help ensure that appropriate interventions are chosen for its prevention. To the same end, they should consider the mechanisms by which relevant risk factors may have led to violent behavior. In the first four studies (chapters 2-5), the following risk factors were identified as the most useful targets for interventions: delusions, impulsivity, substance use, poor insight and treatment nonadherence. With the exception of treatment nonadherence, interventions with a strong evidence base are available for each of these risk factors.

The findings from the second study (chapter 3 ) suggest that risk assessment and interventions should target any substance use. This contrasts with current practice. Almost all risk assessment tools contain only a general item for SUD or exclude certain substances. Interventions 
focus on SUD but may also assist in the prevention of violent behavior in patients with subthreshold use.

The third study (chapter 4) showed that cognition has little relevance to violence in people with psychotic disorders. Although caution is warranted in extrapolating results from other populations, the same conclusion may be drawn from the fifth study (chapter 6) regarding regional GM volume. Both studies have important implications for legal practice. The use of neuroscientific evidence in criminal proceedings has grown sharply in Western countries, including the Netherlands, over the past two decades. However, the results suggest that - in the absence of gross pathology - it is inappropriate to explain violent crimes committed by defendants with psychotic disorders from neuropsychological test scores or the size of brain regions.

The third study (chapter 4) also has clinical implications in that cognitive deficits are likely not useful for the prediction or prevention of violence in psychotic disorders. The small effect sizes suggest that adding items for cognitive functions to risk assessment tools would marginally improve their performance at best. Similarly, psychological interventions designed to improve cognitive functions should not be expected to prevent violent behavior in many patients.

From the results of the fourth study (chapter 5), it follows that clinicians should establish whether patients became violent before or after their first psychotic episode when assessing them for risk and suitable interventions. None of the risk assessment tools currently available makes this distinction. With respect to interventions, both VBO and VAO may be prevented by improving impulse control. Additional targets for the prevention of VAO are SUD, delusions and lack of illness insight.

Many people will benefit if violence in psychotic disorders is more effectively prevented. First, and perhaps most obviously, less harm will come to victims. The emotional impact of caring for a loved one or treating a patient who is violent should also not be underestimated. The fact that victims are often family members or mental healthcare professionals makes this all 
the more poignant. Second, it should reduce the stigmatization of people with psychotic disorders. This is important, as stigma is an obstacle to their treatment and hampers social functioning. Of course, significant improvements in prevention are not achieved quickly. Nor is it acceptable to control news coverage of violence by psychotic individuals or easy to tackle their stereotyped portrayal as violent in books or movies. Nevertheless, public awareness campaigns providing accurate information about the prevalence and causes of violence in psychotic disorders may help dispel common misconceptions. Finally, it will reduce the economic costs to society at large. These costs are substantial. Violence costs the global economy about $€ 1.40$ trillion every year in medical care, productivity losses and criminal justice processes. Assuming a population attributable fraction of $5 \%$, this gives a crude estimate of $€ 70$ billion for the same costs of violent behavior in psychotic disorders. 


\section{Nederlandse samenvatting (Dutch summary)}

\section{Achtergrond}

Psychotische stoornissen zijn een groep psychische aandoeningen waarbij waarneming, denken, emotie en gedrag ernstig ontregeld zijn. Voorbeelden zijn schizofrenie, schizoaffectieve stoornis en waanstoornis. Symptomen bestaan onder meer uit waanideeën, hallucinaties en verwardheid. Hoewel ze minder dan $1 \%$ van de bevolking treffen, behoren psychotische stoornissen tot de meest slopende en duurste psychische aandoeningen.

In de afgelopen 30 jaar heeft onderzoek een robuust verband vastgesteld tussen psychotische stoornissen en gewelddadig gedrag. Er wordt geschat dat tussen de $10 \%$ en $25 \%$ van patiënten tenminste eenmaal in hun leven geweld vertoont. Daarmee is de kans op geweld in deze populatie tot zeven keer hoger dan in de algemene populatie. De toerekenbare fractie voor de bevolking is ongeveer $5 \%$.

Er zijn echter vijf belangrijke vragen. Ten eerste is onduidelijk wat de oorzaken zijn voor geweld bij mensen met een psychotische stoornis. De voornaamste reden hiervoor is dat onderzoek overwegend retrospectief is gedaan. Ten tweede is onbekend welke invloed de psychofarmacologische eigenschappen van drugs en niet-problematisch drugsgebruik hebben op het ontstaan van geweld. Dit zijn klinisch relevante vragen, aangezien niet alle drugs samenhangen met geweld in de algemene populatie en mensen met een psychotische stoornis zeer gevoelig zijn voor hun effecten. De weinige studies naar cannabis en stimulerende middelen hebben gebruik gemaakt van indirecte uitkomstmaten, zoals vijandigheid en agressie, terwijl geen enkele studie heeft gekeken naar verdovende of bewustzijnsveranderende middelen. Ten derde is de rol van cognitie onduidelijk. Mensen met een psychotische stoornis hebben vaak cognitieve problemen. Bovendien worden zulke problemen in verband gebracht met geweld in de algemene populatie en risicofactoren daarvoor in patiënten, zoals werkloosheid, drugsmisbruik en gedwongen ziekenhuisopnames. Een groot nadeel van relevant onderzoek 
is dat het cognitieve functies heeft samengevoegd of alleen heeft gekeken naar kleine selecties daarvan. Ten vierde bestaat er discussie over de vraag of risicofactoren verschillen afhankelijk van of geweld plaatsvindt voor- (GVP) of nadat (GNP) de psychotische stoornis zich manifesteert. Het is aannemelijk dat risicofactoren voor GVP vergelijkbaar zijn met die voor geweld in de algemene populatie en dat risicofactoren voor GNP vooral ziektegerelateerd zijn. Zover ik weet zijn deze hypothesen nog nooit getest. Ten slotte is er de vraag of er een verband bestaat tussen hersenafwijkingen en geweld in psychotische stoornissen. Verlies van grijze stof, bestaande uit de cellichamen van neuronen en hun uitlopers, is in patiënten wijdverbreid en het sterkst in de frontale en temporale kwabben, hippocampus en amygdala. Er wordt verondersteld dat dit gewelddadig gedrag kan veroorzaken via verstoringen in emotieregulatie, het leren van ervaring en besluitvorming. Studies in de algemene en andere psychiatrische populaties kunnen in dezen ook informatief zijn, maar inconsistente resultaten en variatie in anatomische nomenclatuur bemoeilijken interpretatie.

\section{Voornaamste bevindingen}

Dit proefschrift heeft tot doel om de huidige kennis van gewelddadig gedrag bij mensen met een psychotische stoornis te vergroten. Hiertoe zijn vijf studies uitgevoerd. Allereerst heb ik mogelijke causale paden beschreven tussen geweld en de meest gerepliceerde risicofactoren, waaronder kindermishandeling, psychotische symptomen, middelenmisbruik en beperkt ziekte-inzicht (hoofdstuk 2). Risicofactoren komen vaak gelijktijdig voor en staan in wisselwerking met elkaar.

Vervolgens heb ik drie studies uitgevoerd met data van het project Genetic Risk and Outcome of Psychosis (GROUP). De steekproef bestond uit 891 patiënten met een psychotische stoornis, gerekruteerd in 40 Nederlandse instellingen voor geestelijke gezondheidszorg. De eerste studie (hoofdstuk 3) gebruikte ook data van 921 personen die zich met een eerste psychose hadden gemeld bij vijf vroegtijdige interventiediensten in Engeland. Zij namen deel aan het project National Evaluation of the Development and Impact of Early Intervention Services 
(NEDEN). Door de data van GROUP en NEDEN samen te voegen, heb ik de effecten van dagelijks en niet-dagelijks gebruik op gewelddadig gedrag kunnen onderzoeken voor cannabis en stimulerende, verdovende en bewustzijnsveranderende middelen. Het bleek dat, ongeacht de frequentie, elk van deze soorten drugs het risico op geweld verhoogde. De effecten waren het kleinst voor cannabis en het grootst voor stimulerende middelen. Mogelijke redenen waarom drugsgebruik geweld veroorzaakt zijn ontremming, verergering van psychotische symptomen, het plegen van vermogenscriminaliteit om een verslaving in stand te houden en betrokkenheid bij illegale drugshandel.

In de tweede studie (hoofdstuk 4) heb ik de verbanden onderzocht tussen neuropsychologische testprestaties en geweld voor een uitgebreide reeks cognitieve functies. Deze omvatten onder meer het probleemoplossend vermogen, cognitieve flexibiliteit, planning en het vermogen om zich te verplaatsen in de gedachten en gevoelens van anderen. Voor iedere test verklaarde geweld minder dan $1 \%$ van de variantie in de scores. Het lijkt er dus op dat cognitieve problemen een verwaarloosbare rol spelen bij de ontwikkeling van geweld in mensen met een psychotische stoornis.

In de derde studie (hoofdstuk 5) heb ik risicofactoren geïdentificeerd voor geweld dat begint voor de eerste psychose (GVP) en geweld dat daarna begint (GNP). Zowel patiënten met GVP als patiënten met GNP bleken impulsiever in vergelijking met niet-gewelddadige patiënten. Daarnaast hadden patiënten met GNP vaker drugs misbruikt, ernstiger waanvoorstellingen en minder ziekte-inzicht dan niet-gewelddadige patiënten. Deze resultaten doen vermoeden dat GVP en GNP beide ziektegerelateerd zijn. Impulsiviteit is namelijk een kenmerk van psychotische stoornissen en, relevant voor GVP, ontstaat dikwijls voor de eerste psychose. Impulsiviteit kan tot geweld leiden wanneer iemand onvoldoende nadenkt over de mogelijke gevolgen van zijn of haar handelen. Wanen kunnen gewelddadig gedrag motiveren, met name als ze boosheid opwekken. Drugsmisbruik en slecht ziekte-inzicht zijn sterke voorspellers voor therapieontrouw.

Tot slot heb ik een systematisch literatuuronderzoek uitgevoerd naar het verband tussen lokaal grijze stof volume en geweld (hoofdstuk 6). Deze bevatte 35 neuroimaging studies die 
informatie verschaften voor 86 hersendelen in bij elkaar 1,288 proefpersonen. De pariëtale kwab was het enige gebied waarvoor meer dan de helft van de studies verminderingen in grijze stof rapporteerden. Deze waren echter verspreid over subregio's. Een meta-analyse liet geen clusters zien van volumereducties. Op basis van deze bevindingen concludeerde ik dat geweld etiologisch te complex is om te vangen met een enkele biomarker.

\section{Aanbevelingen}

De bevindingen in dit proefschrift hebben meerdere klinische en juridische implicaties. De eerste studie (hoofdstuk 2) maakt duidelijk dat behandelaren patiënten moeten screenen op een breed scala aan risicofactoren voor geweld, zodat geschikte interventies worden gekozen ter voorkoming daarvan. Ook moeten ze voor hetzelfde doel nagaan welke mechanismen hebben geleid tot gewelddadig gedrag. De eerste vier studies (hoofdstukken 2-5) identificeerden de volgende risicofactoren als de meest bruikbare doelwitten voor interventies: wanen, impulsiviteit, drugsgebruik, beperkt ziekte-inzicht en therapieontrouw.

De resultaten van de tweede studie (hoofdstuk 3) brengen met zich mee dat risicotaxatie en preventie zich moeten richten op ieder drugsgebruik. Dit staat in contrast met de huidige praktijk. Bijna alle risicotaxatie-instrumenten bevatten één algemeen item voor drugsmisbruik of laten bepaalde drugs weg. Hoewel interventies hoofdzakelijk worden gebruikt voor problematisch drugsgebruik, helpen zij mogelijk ook bij het voorkomen van gewelddadig gedrag in patiënten met niet-problematisch drugsgebruik.

Uit de derde studie (hoofdstuk 4) bleek dat cognitie nagenoeg geen rol speelt bij het ontstaan van geweld in psychotische stoornissen. Hoewel voorzichtigheid geboden is bij het extrapoleren van resultaten naar andere populaties, kan dezelfde conclusie worden getrokken uit de vijfde studie (hoofdstuk 6) met betrekking tot regionaal hersenvolume. Beide studies hebben juridische implicaties. Het gebruik van neurowetenschappelijk bewijs in strafzaken is de afgelopen twee decennia sterk gegroeid in Nederland. De resultaten suggereren echter dat het 
ongepast is om, in de afwezigheid van grove pathologie, geweldsmisdrijven door beklaagden met een psychotische stoornis te verklaren op basis van neuropsychologische testscores of de grootte van hersengebieden.

Een klinische implicatie van de derde studie (hoofdstuk 4) is dat cognitieve problemen nauwelijks nut hebben voor het voorspellen of voorkomen van geweld bij mensen met een psychotische stoornis. Gezien de kleine effecten zullen items voor cognitieve functies de prestaties van risicotaxatie-instrumenten marginaal verbeteren. Evenmin kan worden verwacht dat interventies die cognitieve functies beogen te verbeteren geweld voorkomen in veel patiënten.

Uit de resultaten van de vierde studie (hoofdstuk 5) volgt dat, bij het inschatten van het risico op toekomstig geweld en het kiezen van geschikte interventies, behandelaren moeten vaststellen of patiënten gewelddadig zijn geweest voor hun eerste psychose of alleen daarna. Geen van de huidige risicotaxatie-instrumenten maakt dit onderscheid. Zowel GVP als GNP wordt mogelijk voorkomen door de impulscontrole van patiënten te verbeteren. De preventie van GNP dient zich ook te richten op drugsmisbruik, wanen en gebrekkig ziekte-inzicht.

Veel mensen hebben er baat bij als geweld in psychotische stoornissen vaker wordt voorkomen. Ten eerste, en het meest voor de hand liggend, zal er minder leed zijn voor slachtoffers. Aangezien slachtoffers vaak familieleden of zorgmedewerkers zijn, moet ook de emotionele impact van het zorgen voor een dierbare of patiënt die met geweld reageert niet vergeten worden. Ten tweede gaat het de stigmatisering tegen van mensen met een psychotische stoornis. Dit is belangrijk, omdat stigma een belemmering vormt voor hun behandeling en sociaal functioneren. Uiteraard worden betekenisvolle verbeteringen op het gebied van preventie niet snel bereikt. Daarnaast is het moeilijk om de stereotypering van patiënten in de media en fictie aan te pakken. Toch kunnen campagnes, die het publiek correct informeren over de prevalentie en oorzaken van geweld bij mensen met een psychotische stoornis, helpen om misvattingen weg te nemen. Ten slotte verlaagt het de economische kosten voor de samenleving. Deze kosten zijn aanzienlijk. Geweld kost wereldwijd ieder jaar ongeveer $€ 1.40$ 
biljoen aan medische zorg, productiviteitsverlies en strafrechtelijke processen. Ervan uitgaande dat $5 \%$ van al het geweld kan worden toegeschreven aan mensen met een psychotische stoornis, dan geeft dit $€ 70$ miljard als een ruwe schatting van de daaraan gerelateerde kosten. 


\section{Publications arising from this dissertation}

Chapter 2

Lamsma J, Harte JM. Violence in psychosis: conceptualizing its causal relationship with risk factors. Aggress Violent Behav 2015; 24: 75-82.

Chapter 3

Lamsma J, Cahn W, Fazel S, GROUP and NEDEN investigators. Use of illicit substances and violent behavior in psychotic disorders: two nationwide case-control studies and metaanalyses. Psychol Med 2020; 50: 2028-2033.

\section{Chapter 4}

Lamsma J, Cahn W, Fazel S, GROUP investigators. Cognition and violent behavior in psychotic disorders: a nationwide case-control study. Schizophr Res Cogn 2020; 19: 100166.

\section{Chapter 5}

Lamsma J, Cahn W, Harte JM, GROUP investigators. Risk factors for violence before and after the onset of schizophrenia spectrum disorder: a Dutch nationwide case-control study. Submitted for review.

\section{Chapter 6}

Lamsma J, Mackay C, Fazel S. Structural brain correlates of interpersonal violence: systematic review and voxel-based meta-analysis of neuroimaging studies. Psychiatry Res Neuroimaging 2017; 30: 69-73. 

